University of Tennessee Health Science Center

UTHSC Digital Commons

$12-1999$

\title{
Genetic Control of Eye Development, a Case Study Focused on the Murine Mutation Belly Spot and Tail (BST)
}

Qing Tang

University of Tennessee Health Science Center

Follow this and additional works at: https://dc.uthsc.edu/dissertations

Part of the Genetic Processes Commons, Medical Genetics Commons, and the Medical Neurobiology Commons

\section{Recommended Citation}

Tang, Qing , "Genetic Control of Eye Development, a Case Study Focused on the Murine Mutation Belly Spot and Tail (BST)" (1999). Theses and Dissertations (ETD). Paper 259. http://dx.doi.org/10.21007/ etd.cghs.1999.0314.

This Dissertation is brought to you for free and open access by the College of Graduate Health Sciences at UTHSC Digital Commons. It has been accepted for inclusion in Theses and Dissertations (ETD) by an authorized administrator of UTHSC Digital Commons. For more information, please contact jwelch30@uthsc.edu. 


\title{
Genetic Control of Eye Development, a Case Study Focused on the Murine Mutation Belly Spot and Tail (BST)
}

\begin{abstract}
Development of the eye is controlled by a network of genes, often conserved, that regulate the timing and location of cellular differentiation. One approach to understanding this network of genes and their interaction is to focus on mutations, spontaneous or induced, that predictably disrupt the proper function of such networks, and by examining the effect of such disruption on the function of other genes.

The Belly spot and tail (Bst) semi-dominant mutation, mapped to mouse Chromosome 16, leads to developmental defects of the eye, skeleton, and coat pigmentation. In the eye, the mutant phenotype is characterized by the presence of retinal colobomas, a paucity of retinal ganglion cells, and axon misrouting. The severity of defects in the Bst/+ retina is variable among individuals and is often asymmetric. In order to determine the role of the Bst locus during retinal morphogenesis, we searched for the earliest observable defects in the developing eye. We examined the retinas of Bst/+ and $+/+$ littermates from embryonic day 9.5 (E9.5) through E13.5, and measured retinal size, cell density, cell death, mitotic index and cell birth index. We have found that development of the Bst/+ retina is notably dilatory by as early as E10.5. The affected retinas are smaller than their wildtype counterparts, and optic fissure fusion is delayed. In the mutant, there is a marked lag in the exit of retinal cells from the mitotic cycle, even though there are no observable differences in the rate of cellular proliferation or cell death between the two groups. We hypothesize that Bst regulates retinal cell differentiation, and that variability of structural defects in the mutant, such as those affecting optic fissure fusion, is a reflection of the extent of developmental delay brought about by the Bst mutation.
\end{abstract}

In an effort to determine the role of Bst within the network of genes controlling eye development, we examined the effects of Bst in relation to two genes believed to regulate eye development during the same developmental period (E9-13): the boundary gene Pax2, which plays a role during optic stalk fissure fusion, and the proneural gene Hes1, which has been shown to regulate retinal ganglion cell differentiation. We cross-mated hemizygous Bst/+ mutant mice with hemizygous $\mathrm{Krd} /+$ (kidney and retinal defects, in which Pax2 is deleted) mice and Hes1 +/- knockout mice to produce Bst/+ Krd/+ and Bst/+ Hes1 +/- compound heterozygous mutants. We find that there is marked ectopic expression of Pax2 protein in the Bst/+ retina, and a potentiation of the retinal defects in Bst/+ Krd/+ compound mutant offspring compared to the parental mutant phenotypes; which indicates that the Bst mutation could have resulted in a loss of positional cues for subsequent morphogenetic events, such as those dependent upon Pax2 expression. In contrast, the level of Hes1 mRNA in the Bst/+ mutant appears normal. In the Bst/+ Hes1 +/- compound mutant offspring, there are fewer instances of ocular defects compared to those of Bst/+ Hes1 +/+ littermates. Given that Hes1 is believed to be an inhibitor of cellular differentiation, the partially improved Bst/+ Hes1 +/- retinal phenotype is consistent with the hypothesis that the effects of the two mutant alleles are antagonistic.

Taken together, our results suggest that the Bst locus is involved in the regulation of cellular differentiation during early eye development.

\section{Document Type}

Dissertation

Degree Name

Doctor of Philosophy (PhD) 


\section{Program}

Anatomy and Neurobiology

Research Advisor

Daniel Goldowitz, Ph.D.

\section{Subject Categories}

Genetic Processes | Medical Genetics | Medical Neurobiology | Medical Sciences | Medicine and Health Sciences 


\title{
Genetic control of eye development, a case study focused on the murine mutation Belly spot and tail (Bst)
}

\author{
A Dissertation \\ Presented for \\ The Graduate Studies Council \\ The University of Tennessee, Memphis \\ In Partial Fulfillment \\ Of the Requirements for the Degree \\ Doctor of Philosophy \\ From the University of Tennessee
}

By

Qing Tang

December, 1999 


\section{Acknowledgments}

"You missed your calling", well-meaning people have been known to tell me. After 7 years of taxpayer-sponsored education, and facing imminent dismissal from student tenure, those words can have unsettling implications. I have learned a lot from the people here at UT, not just in terms of how to think scientifically, but also in terms of the intangibles of research. I have come to realize that curiosity may put one on a path to a sea of knowledge, but the trip would be short-lived if one is not fueled by a great deal of zeal and perseverance. In the course of my apprenticeship, I have become well acquainted with my limitations; which is why I appreciate all the more the droves of people who have pushed and carried me to where I am today. To you I owe a debt of gratitude that would be harder to repay than the years of tuition waivers and stipends combined.

The UT front, in no particular order of importance:

Dr. Dan Goldowitz, who has managed to mentor me in spite of my efforts at undermining his plans, for whom my admiration is both intellectual and personal. My committee members, Drs. Robert Williams, Eldon Geisert, D.J. Surmeier, and John Cox, whose patience and input have always been deeply appreciated.

Dr. Kristin Hamre, who has been a constant and valued source of advice on a multitude of topics, including graduate school survival and gun control.

Mr. Richard Cushing and Ms. Kathy Troughton, without whose skilled technical assistance the project may well have become trans-millenial.

Dr. Guo-Ming Zhou, whose verbal encouragements are backed by active help.

Dr. Denny Rice, Mr. Alan Ezer, and Ms. Tricia Jensen, with whom I have had the pleasure to share a lab bench as well as lunch buffets at Dragon China. You have all enriched my experience as a student and as a person.

The home front:

My wife Siew and daughter Hera, whom I love and cherish, and who deserve a punitive award for pain and suffering.

My parents, who have been and will always be twice of me.

My grandparents, who showed me how dignity can make hardship bearable.

My uncle, who sent me to a good school twenty years ago without expectations, and still has not changed his mind.

My junior brother, who is a constant reminder of how else I might have turned out. Chapter 4 is modified from Tang et al., 1999, and included in the dissertation in accordance with copyright transfer agreement (item A-4) from Academic Press. Fundus camera photographs used in Fig. 3.3 and Fig. 5.4 are by Norm Howes. 


\section{Abstract}

Development of the eye is controlled by a network of genes, often conserved, that regulate the timing and location of cellular differentiation. One approach to understanding this network of genes and their interaction is to focus on mutations, spontaneous or induced, that predictably disrupt the proper function of such networks, and by examining the effect of such disruption on the function of other genes.

The Belly spot and tail (Bst) semi-dominant mutation, mapped to mouse Chromosome 16, leads to developmental defects of the eye, skeleton, and coat pigmentation. In the eye, the mutant phenotype is characterized by the presence of retinal colobomas, a paucity of retinal ganglion cells, and axon misrouting. The severity of defects in the $B s t /+$ retina is variable among individuals and is often asymmetric. In order to determine the role of the $B s t$ locus during retinal morphogenesis, we searched for the earliest observable defects in the developing eye. We examined the retinas of $B s t /+$ and +/+ littermates from embryonic day 9.5 (E9.5) through E13.5, and measured retinal size, cell density, cell death, mitotic index and cell birth index. We have found that development of the $B s t /+$ retina is notably dilatory by as early as E10.5. The affected retinas are smaller than their wildtype counterparts, and optic fissure fusion is delayed. In the mutant, there is a marked lag in the exit of retinal cells from the mitotic cycle, even though there are no observable differences in the rate of cellular proliferation or cell death between the two groups. We hypothesize that $B s t$ regulates retinal cell differentiation, and that variability of structural defects in the mutant, such as those affecting optic fissure fusion, is a reflection of the extent of developmental delay brought about by the Bst mutation.

In an effort to determine the role of $B s t$ within the network of genes controlling eye development, we examined the effects of $B s t$ in relation to two genes believed to regulate eye development during the same developmental period (E9-13): the boundary gene $P a x 2$, which plays a role during optic stalk fissure fusion, and the proneural gene Hes 1, which has been shown to regulate retinal ganglion cell differentiation. We crossmated hemizygous Bst/+ mutant mice with hemizygous $\mathrm{Krdl}+$ (kidney and retinal defects, in which Pax2 is deleted) mice and $\mathrm{Hesl}^{+/-}$knockout mice to produce Bst/+ $\mathrm{Krdl} /+$ and $B s t /+\mathrm{Hesl}^{+/-}$compound heterozygous mutants. We find that there is marked ectopic expression of $\operatorname{Pax} 2$ protein in the $B s t /+$ retina, and a potentiation of the retinal defects in $B s t /+K r d /+$ compound mutant offspring compared to the parental mutant phenotypes; which indicates that the Bst mutation could have resulted in a loss of positional cues for subsequent morphogenetic events, such as those dependent upon 
Pax2 expression. In contrast, the level of Hesl mRNA in the Bst/+ mutant appears normal. In the Bst/+ Hesl ${ }^{+-}$compound mutant offspring, there are fewer instances of ocular defects compared to those of Bst $/+\mathrm{Hesl}^{+/+}$littermates. Given that Hesl is believed to be an inhibitor of cellular differentiation, the partially improved Bst/+ HesI ${ }^{+/-}$retinal phenotype is consistent with the hypothesis that the effects of the two mutant alleles are antagonistic.

Taken together, our results suggest that the Bst locus is involved in the regulation of cellular differentiation during early eye development. 


\section{Table of Contents}

Chapter 1. The study of eye development.

1.1 Why study eye development in the mouse? 10

1.2 What is known about the eye? 11

1.3 The goal of the dissertation project. 13

1.4 Theme and layout of the dissertation. 14

Chapter 2. Origin and development of the eye.

2.1 Introduction. 16

$\begin{array}{ll}2.2 & \text { Origin of the eye. } \\ & 17\end{array}$

2.2.1 Origin of vision. 17

2.2.2 The many forms of eyes. 21

2.2.2.1 Simple eyes. 21

2.2.2.2 Compound eyes. 24

2.3 Eye development. 27

2.3.1 The sequence of events during eye development. 27

2.3.1.1 The genesis of the Drosophila eye. 28

2.3.1.2 The developing vertebrate eye. 29

2.3.2 The genetic control of eye development. 32

2.3.3 The eye genes. 35

2.3.3.1 The early patterning genes. 36

2.3.3.2 The cellular specification and differentiation genes $\quad 44$

2.4 Conclusions. 49 16 17 1

7

8

9

5

Chapter 3. Bst, a semi-dominant mutation causing retinal defects.

3.1 Introduction. $\quad 51$

3.2 What is $B s t$ ?

3.3 The Bst retina. $\quad 57$

3.4 The study of $B s t$ 's role during eye development. 58

Chapter 4. Disrupted retinal development in the embryonic Bst mutant mouse.

4.1 Introduction. $\quad 60$

4.2 Materials and methods. 61

4.2.1 Animals. 61

4.2.2 Embryo collection. $\quad 62$

4.2.3 Histological methods. $\quad 62$

4.2.4 BrdU-labeling of mitotic cells. 63 
4.2.5 TUNEL analysis of dying cells. 65

4.2.6 Analysis of retinal morphology, cell density, 66 and proliferative index.

$\begin{array}{ll}\text { 4.2.7 Image capture and processing } & 67\end{array}$

$\begin{array}{lll}4.3 & \text { Results. } & 67\end{array}$

4.3.1 Gross observation of embryonic development. 67

4.3.1.1 E9.5.

4.3.1.2 E10.5. 68

4.3.1.3 E11.5. 68

4.3.1.4 E12.5.

4.3.1.5 E13.5. 70

4.3.2 Retinal development. $\quad 70$

4.3.2.1 Bst/+ retinas are slightly undersized and fuse late $\quad 70$ during development.

4.3.2.2 Bst/+ retinas have normal cell density. $\quad 72$

4.3.2.3 Cellular proliferation rate is normal in the Bst/+. $\quad 74$

4.3.2.4 Cell death is normal in the Bst/+.

4.3.2.5 The exit of cells from the cell cycle is delayed in the 76 $B s t /+$ retina.

4.3.2.6 Retinal development is asymmetrically disturbed in $\quad 77$ the $B s t /+$ mutant.

4.4 Discussion. $\quad 80$

4.4.1 Bst plays a role during early retinal development, $\quad 80$ independent of cell proliferation and cell death.

4.4.2 Bst may regulated cell differentiation. 81

4.4.3 The Bst mutation likely disrupts timing and coordination of 82 developmental events during retinal morphogenesis.

\section{Chapter 5. Bst is a regulator of early retinal development: evidence} of interaction between Bst, Pax2 and Hes1.

5.1 Introduction. $\quad 84$

5.2 Materials and methods. $\quad 86$

5.2.1 Animals and genotyping. $\quad 86$

5.2.2 Adult retina collection and analysis. 88

5.2.3 Quantification of axon number in the optic nerve. 88

$\begin{array}{ll}\text { 5.2.4 Embryo collection. } & 90\end{array}$

5.2.5 RNA collection and Hes I northern hybridization. $\quad 90$

5.2.6 Pax2-immunohistochemistry. 91

$\begin{array}{lll}5.3 & \text { Results. } & 92\end{array}$

5.3.1 Bst and Pax2 interactions. $\quad 92$

5.3.1.1 Pax2 expression is abnormal in the developing $\quad 92$ $B s t /+$ retina. 
5.3.1.2 The survival of $B s t /+K r d /+$ compound mutant offspring is compromised.

5.3.1.3 The $B s t /+K r d /+$ compound mutation results in increased incidence of eye defects.

5.3.1.4 The Bst/+ Krd/+ compound mutation exacerbates retinal and optic nerve atrophy.

5.3.2 The interactive effects of $B s t$ and Hes 1 mutations.

102

5.3.2.1 Hes 1 expression is likely normal in the Bst/+.

102

5.3.2.2 The combined effect of Hesl and Bst mutation on 103 survival is complex.

5.3.2.3 Bst/+ HesI ${ }^{+/-}$compound mutation results in mild $\quad 104$ ocular defects.

5.3.2.4 Bst/+ Hes ${ }^{+/-}$compound mutants have relatively $\quad 104$ healthy retinas and optic nerves.

5.4 Discussion.

5.4.1 Bst disrupts gene expression in the retina.

108

5.4.2 The Bst mutation is probably not a defect in Hesl expression.

5.4.3 Bst and Hes I may be antagonistic regulators of

109 differentiation.

\section{Chapter 6. Conclusions and interpretations.}

6.1 Result summary and discussion. 111

6.2 The effects of $B s t$ on eye development likely predate 112 optic cup formation.

6.3 The meaning of the developmental delay and phenotypic 113 variability.

6.4 The nature of the Bst gene. 117

$\begin{array}{ll}\text { List of References. } & 120\end{array}$

$\begin{array}{ll}\text { Vita. } & 138\end{array}$ 


\section{List of Tables}

Table

3.1 Bst adult phenotype.

3.2 Retinal defects and associated mutations.

4.1 Embryonic Bst litter composition.

5.1 Compound mutants litter composition.

5.2 Incidence of pupillary reflex defects in mutant mice.
Page 55

56

68

94

97 


\section{List of Figures}

Figure Page

2.1 The developing vertebrate eye. 30

2.2 The expression patterns of regulatory genes during eye development. 39

3.1a The range of phenotypes in the Bst/+ mice.

$3.1 \mathrm{~b}$ Optic nerve defects in the Bst/+ mutant mouse. 53

3.2 Polydactyly and exencephaly in the $B s t /+$ mutant mouse. 53

3.3 Fundus camera view of the $B s t /+$ retina. $\quad 57$

3.4 Optic fissure fusion anomaly in the $B s t /+$ retina. $\quad 58$

4.1 Bst/+ embryos exhibit developmental defects. $\quad 69$

4.2 Retinal growth and cellular proliferation in Bst/+ from E10.5 to E13.5. 71

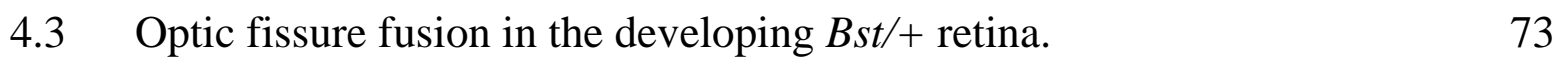

$\begin{array}{lll}4.4 & \text { Retinal growth and cellular proliferation profiles. } & 75\end{array}$

4.5 Cell death in the developing $B s t /+$ retina. 76

4.6 Delayed neuronogenesis in the $B s t /+$ retina. 78

5.1 Electronmicrographs of the optic nerve. $\quad 89$

5.2 Pax2 expression in the developing Bst/+ retina. 93

5.3 The developing $B s t /+K r d /+$ compound mutant embryos. 95

5.4 Fundus camera view of the $K r d /+$ retina. 96

5.5 Disrupted retinal development in the Bst, Krd, and Hes1 99 compound mutant mice.

5.6 The retinal and optic nerve characteristics of Bst and $K r d$ mutant mice. 100

5.7 Hesl RNA expression in the Bst/+ mutant mouse. 103

5.8 The developing Hes ${ }^{+/}$embryos. 105

5.9 The retinal and optic nerve characteristics of Bst and Hes Imutant mice. 106

6.1 Hypothetical relationship between cellular differentiation, 115 embryonic fissure formation and fissure fusion. 
Chapter 1 . The study of eye development.

\subsection{Why study eye development in the mouse?}

Vision, the ability to detect light and extract the information it contains, is the combined product of photoreception and signal processing. In the vertebrate eye, the light detecting and signal transduction processes begin at the outer segment of individual photoreceptors, then continue through the network of receptors in the outermost layer of the retina, and proceeds through successively higher processing areas of the visual system (i.e., the inner layers of the retina, the visual nuclei in the thalamus, the primary visual cortex, and associated cortical areas). For humans, vision is one of the most dependedupon senses, and has been the subject of marvel and bewilderment for biologists and poets alike. Over the past two centuries, investigators have been able to elucidate much of the mechanical aspects of the visual apparatus, as well as the physical basis behind light detection and signal transduction. However, many fundamental questions remained unaddressed or inadequately addressed until only decades ago. Notably, our understanding of the origin of the eye and the regulation of its development has traditionally relied upon anecdotal evidence and educated guesses, where precise data have been difficult to obtain. Recent advances in the field of genetics and molecular technology have provided us with powerful tools to examine many of these previously difficult to address questions. Still, the complexity of the genetic mechanisms underlying eye development, and the diversity that exists between the eyes of various organisms, make any attempt to address these questions while relying solely on molecular approaches appear foolish. Indeed, as one observer of trends in biological research points out, the current bias in favor of hypothesis-driven research projects employing the latest molecular techniques, at the expense of traditional "descriptive" studies, may be fundamentally misguided, as knowledge gained through the test of hypotheses that are based on an incomplete understanding of the natural history of a biological phenomenon not only contributes little to the understanding of the phenomenon, but may even mislead the investigator into making wrong assumptions that could impede progress (Rajan, 1999). Therefore, to meaningfully examine a particular aspect of eye development involving genetic factors, we must first have an understanding of the anatomical events through which we can adequately and correctly define the developmental problem. To this end, we should seek to broaden our perspective by drawing upon the pool of knowledge gained through the study of eye development in a variety of species. 
Comparative anatomical studies have shown that metazoan organisms which differ widely in appearance could share many similarities in their body plans, and the information extracted from one can often be applied to others with surprising predictability. For instance, all 60,000 members of the phylum Chordata, from lampreys to humans, develop from radial cleavage of blastomeres, and invariably display bilateral symmetry. Their organs form from three germ layers, which give rise to superficial nervous and muscular organs, and internal digestive and reproductive organs. All chordates exhibit metamerism and regional differentiation in the organization of their body plans (Hickman, 1961). With few exceptions, these features are also found in other deuterostomes. Beyond that, the layout and development of the three germ layers and the metameric organization of axial structures in deuterostomes have many similarities with those of protostomes (Gilbert, 1985). Recent molecular evidence also shows that among animals of different phyla, the genetic control of organogenesis is an area where commonality between species can often be found. Genes containing highly similar sequences exist in organisms as different as Drosophila and humans. These genes code for nearly identical molecules that have been found to play similar regulatory roles during the development of vital organs. A number of these genes that have important functions during eye development are discussed in Chapter 2 . Between phyletically close species such as human and the mouse, the elucidation of a given genetic mechanism regulating development in one organism virtually guarantees the understanding of the homologous mechanism in the other.

Consequently, the study of eye development in non-human animals such as the mouse, while a satisfying end in itself, is also an invaluable tool with which we could gain insights into the workings of our own visual system - How does it work? And perhaps more interestingly - How did it come about?

\subsection{What is known about the eye?}

With the introduction of the compound microscope at the end of 16th century and the subsequent development of more refined histological means and methods, casual observation of organs and tissues characterizing the early biological inquiries gradually gave way to rigorous and methodical scientific undertakings. Not surprisingly, the eye has been the subject of intensive studies using the most modern methods. Its accessibility coupled with its important function guaranteed its attractiveness to researchers. Much of the earlier work was necessarily descriptive and focused on the anatomy of the eye. 
Through these pioneering investigations, many varieties of eye forms from all manners of organisms had been recognized and recorded, and these became the basis for more in depth comparative anatomical analyses when better tools became available (Walls, 1942). Concurrently, by examining the eyes of developing embryos, early investigators such as Cajal were able to trace the sequence of events which lead to the formation of the eye, and describe the embryonic tissues which give rise to the later structures (Froriep, 1906). Other investigators who are interested in the regulation of organ development were able to demonstrate that the eye and its associated structures arise through a series of inductive events occurring in sequence from progressively differentiated tissues (Harrison, 1920; Spemann, 1938). During the early part of 20th century, the introduction of electrophysiological techniques and the use of radioactive tracers permitted researchers to directly examine the function and behavior of living nervous tissues, including the retina, in ever-smaller details. Together, these studies contributed to our understanding of the eye as a light detecting extension of the central nervous system, where the neural retina is formed from a complex and highly hierarchical organization of many types of neurons and glia that, working as a system, converts photon detection into coded electrical signals via intercellular electrical and chemical interactions. The universality of the basic light detecting mechanism notwithstanding, it is evident that animals from different evolutionary lineages have adopted different ways to organize their respective visual organs. These differences reflect the unique survival strategies and evolutionary histories of the organisms, which are discussed in Chapter 2.

Developmental biology did not have the tools to address the molecular mechanisms controlling the formation of these complex structures of the eye until the last few decades. The identification of DNA as the genetic coding molecule in 1953 and the exponential growth of molecular biology that followed permitted developmental biologists to directly examine, and manipulate, the very molecules that control organogenesis, including the genesis of the eye. Animals having a short reproductive cycle and large litters have been favored by geneticists and developmental biologists alike. Principally, the fruitfly Drosophila, the common mouse Mus, as well as Xenopus and zebrafish, have been the subjects of extensive studies, from which much of our current understanding about the genetic regulation of eye development has been gleaned. The detection of naturally-occurring genetic mutants, as well as transgenic manipulations, have been the preferred tools by which "eye genes" have been identified and characterized. The emerging picture of eye development is one of web-like complexity, where every event, however minor it may appear, is related to numerous other events that are part of multiple molecular pathways. At the same time, many genes, such as Pax6 and 
Chx10, appear to perform multiple functions depending on the age and type of the tissues in which they are expressed (see Chapter 2). Conceptually, the genes regulating morphogenesis can be grouped into three functional categories: 1) those which regulate cell proliferation and competence (to respond to differentiation cues), 2) those which determine orientation and positional boundaries, and 2) those which determine cell fate. Every event during development is controlled by a complement of these genes; the process is dynamic and seamless.

The ultra-reductionist approach routinely employed by electrophysiologists to dissect out the behavior of individual receptors and membrane proteins has no equivalent in developmental biology, as it is not yet possible nor meaningful to examine the contribution of a gene dissected from the network of genes with which it interacts. The closest we can come to reducing the complexity in vivo is to study animals that are genetically alike, except for the locus responsible for a developmental problem we are interested in, and use the monochromatic background to "subtract" out the effects of the thousands of genes that do not directly contribute to that particular developmental problem. Extensive inbreeding has produced congenic strains of animals that are closest to this ideal, short of actual clones. The present dissertation project relied as much as possible on the use of such animals in order to characterize the effects of one locus, belly spot and tail (Bst), which affects eye development in the mouse.

\subsection{The goal of the dissertation project.}

The Bst locus has been mapped to mouse Chromosome 16, its mutant allele is responsible for the hypochromatic belly spot and kinked tail phenotype from which it derives its name. The mutation spontaneously occurred in a colony of C57BLKS/J mice. It is transmitted semi-dominantly and is homozygous-lethal on the C57BLKS background (Epstein et al., 1986; Rice et al., 1995).

Briefly, the hemizygous Bst mutants have a kinked tail, a white belly spot, and white feet. A number of the mutants also exhibit pupillary reflex anomaly in the form of decreased or absent pupillary contraction under light stimulation of dark-adapted eyes. The retinal morphology of the mutant mice varies widely, from normal to severe atrophy compounded by structural anomalies; although the affected eyes are never disproportionately undersized (Rice et al., 1997). The optic nerves of the affected eyes are correspondingly hypoplastic. 
The focus of this dissertation project is the events occurring during the developmental period in which the $B s t$ mutation is likely to produce the defects later seen in the adult retinas. This period is between mouse embryonic day 9.5 (E9.5), when the optic vesicle begins to change into optic cup, and E13.5, when the earliest-born retinal neurons have differentiated and the essential retinal structures are laid out. I tested the hypothesis that the normal Bst allele is involved in the fusion of the optic fissure (OF), in conjunction with other genes known to play a part in this process. Specifically, I examined the possible relationship between $B s t$ and $P a x 2$, a known boundary gene that has been shown to affect fissure fusion at the optic stalk (Otteson et al., 1998). I also investigated the possible link between Bst and the mammalian hairy and enhancer of split homologue 1 (Hes 1), a basic helix loop helix (bHLH) transcription factor believed to be involved in the regulation of retinal ganglion cell differentiation (Ishibashi et al., 1995). I hypothesized that Bst is a functional antagonist of Hesl. If Bst is indeed a key element in the gene expression cascade that controls the formation of the retina and the its differentiation, the characterization of Bst could provide much insight into the molecular regulation of vertebrate eye development.

\subsection{Theme and layout of the dissertation.}

The dissertation is presented in five self-contained chapters.

1) Origin and development of the eye.

A survey of the literature on eye evolution and development suggests that there is much in common between widely different visual organs, and that the sharing of common components within diverse developmental pathways may reflect shared descent or convergent evolution. A list of known eye types are given and briefly described. The developmental sequences of two very different eyes are described - those of the Drosophila compound eye and the vertebrate lens-containing eye - showing both differences and similarities. Lastly, a survey of the genes known to be involved in the regulation of retinal development in both the Drosophila and the vertebrate is presented, with emphasis on gene interaction and cross-species homologies. 
2) What is Bst.?

The Bst mutant is introduced as a model for the study of gene regulation of eye development. The general phenotype of the mutation is described and compared with those of other mutations known to disrupt eye development. The rationale for the experiments undertaken is discussed.

3) When and how does Bst affect eye development?

A detailed examination of embryonic eye development in the Bst is performed. The goal is to determine the time frame within which retinal development is disrupted by the Bst mutation, and investigate the likely mechanisms by which such disruption could occur. In particular, the role of optic fissure fusion is discussed within the context retinal development.

4) How does Bst fit into the molecular regulatory pathways controlling eye development?

The relationship between Bst, Pax2, and Hes1 are examined using compound mutant mice. Histological and immunohistochemical methods are used to examine retinal development and gene expression.

5) Result summary and discussion.

The role of $B s t$ during eye development is further speculated upon the context of what is known about other mutant genes that produce similar effects and what has been learned experimentally. The possible molecular identity of $B s t$ is explored. 
Chapter 2. Origin and development of the eye.

\subsection{Introduction.}

Since the beginning of recorded history, humans have sought to understand nature and themselves through rational investigation. One of the most perplexing problems confronting philosophers and scientists through the ages is the origin of living organisms and their propagation. Life seems to arise in its myriad forms out of shapeless and lifeless matters and miraculously proliferate through repetitive cycles. As philosophers searched for the meaning of life through contemplative reflection and discourse, their scientific contemporaries devised increasingly more rigorous and effective methods to study the physical aspects of life, consistently with an eye towards improving the quality of human existence. However, it was not until the middle of the last century that two significant breakthroughs were achieved in the pursuit to understand the propagation of life - the discovery of genetic heredity by Gregor Mendel and the formulation of modern evolutionary theories by Charles Darwin - fueled in large part by advances in mathematics and the physical sciences, which set the stage for many new approaches in biological studies. Mendel's discovery escaped notice for nearly half a century, until later investigators, unaware of his experiments and observations, independently obtained the same insights into the mechanism of inheritance, and subsequently recognized his earlier work. It was not until the mid 1950's that DNA was identified as the molecule that contains the genetic codes.

If one defines evolution as gradual changes in the phenotype of an organism due to natural selection, and if one views the organism as the combined product of its expressed genes, then the concepts of genetic changes and evolution become complementary to one another, such that an evolving organism could be viewed as one in which sufficient changes in the genome have occurred to qualitatively distinguish it from its predecessors. Indeed, the evolving field of modern comparative biology is based on the convention that the "relatedness" between species is a reflection of the level of similarity that exists between the genomes of such species (as opposed to superficial resemblance). Molecular evidence increasingly suggests that there are many shared genes between widely different organisms, and homologous genes typically perform similar functions in different hosts. Therefore, every investigation into the genetic nature of a biological phenomenon, such as organogenesis, would potentially benefit from an examination of its evolutionary implications. 
By definition, development is the creation of order. In effect, the role of any development-related gene is to catalyze or maintain an order. A living organism is the sum total of many such order-creating as well as order-maintaining events. Selective pressure dictates that any aspect of the genome that contributes to the overall competitive fitness of an organism is apt to survive into future generations. Consequently, as organisms evolved apart, those genes which continued to be critical or beneficial to the host's survival and propagation were likely conserved.

In the following sections, I summarize the current understanding with regard to the origin of the many eye forms, followed by a discussion on the genetic regulation of eye development, with emphasis on the interactive nature of the likely regulatory genes. The material discussed suggests that due to the functional importance of vision and the likely early emergence of the visual organs from similar tissues, the molecular regulation of eye development is an area where many common characteristics exist between a wide variety of species. Hence, the continually expanding pool of knowledge concerning eye development in vertebrates and invertebrates alike presents a rich source for clues on how to address questions concerning eye development, regardless of the species in which the questions originate.

\subsection{Origin of the eye.}

\subsubsection{Origin of vision.}

The eye is the culmination of perhaps billions of years of evolution. Its many forms attest to the numerous solutions that exist for the basic and vital functions of discerning physical forms and detecting motion remotely through light perception. With scant exceptions, the molecular mechanism underlying the detection of light is remarkably similar across taxonomic boundaries. The near universality of such a mechanism suggests that either 1) there are very few possible ways to detect light (i.e., only a few organic molecules could respond to the radiation wavelengths within the visible spectrum, and these molecules are found only in certain tissues, thus making it very likely that independently evolved light-detecting organs would share common features), or 2) perhaps most forms of eyes in existence today descended from the same ancestral organ. Evidence suggests that these two concepts are not mutually exclusive, as there are examples that imply common descent as well as convergent evolution in the current assortment of eyes (see later sections). If there is a single common ancestor to all 
existing forms of eyes, it would have to have existed some 1.5 to 2 billion years ago in the Proteozoic era, where fossil records indiacte the emergence of the earliest complex life forms, and well before the pre-Cambrian phyletic explosion some 520 million years ago (Philippe et al., 1994). We can reasonably postulate that the earliest visual organs, which may have been nothing more than aggregates of light-sensitive organelles connected to the neural circuits responsible for feeding, flight or sexual reflexes, conferred such an advantage to the survival and reproduction of their hosts over their blind contemporaries that the latter were competed out of existence.

Common descent is not ascertainable after billions of years of divergent (increasing genotyic differences) and/or convergent (increasing phenotypic similarities from different genotypic starting points) evolution, but it can be inferred via shared genetic regulators and pathways controlling eye development among widely unrelated organisms. Alternatively, the shared genes and regulatory pathways could have existed prior to the independent emergence of various forms of eyes. There are molecular evidence that support this hypothesis as well (see $\operatorname{Pax} 6$ ). At the very least, many genes central to eye development are likely to have existed prior to the phyletic divergence.

The earliest light detecting organelles probably contained visual pigments similar to today's metazoan opsins (Goldsmith, 1990). The opsin molecule has seven transmembrane helices with both extracellular and cytoplasmic loops and is conjugated to a photon-receptive chromophore. When the chromophore is activated by a photon, the opsin changes from the 11-cis form to an all-trans form, which in turn triggers a cascade of enzymatic actions that results in the initiation of the visual signal. The modern Halobacterium has a photosensitive "bacteriorhodopsin" that is structurally similar to metazoan opsins, but which is functionally unique (in the bacteriorhodopsin, the 13th double-bond, rather than the 11th, is changed into the trans form by photon absorption) and shares no sequence homology with metazoan opsins (Goldsmith, 1990). This molecule may be evolutionarily unrelated to the metazoan opsins. The latter can in turn be grouped into several distinct classes according to their wavelength-specific response patterns. The presence of more than two classes of opsins in a visual system forms the physical basis for color vision. Color vision is found in diurnal animals ranging from bees to humans. Amino acid sequence comparisons indicate that all types of cone opsins are derived from the same ancestral form, and that rhodopsin is a permutated form of one of the cone opsins (Okano et al., 1992). Therefore, cone photoreceptors are likely ancestral to rod photoreceptors, and photopic vision probably preceded scotopic vision in the history of eye evolution. It is noteworthy that due to a presumed long-lasting nocturnal lifestyle adopted by the earliest placental mammals, gene-loss may have occurred with 
respect to their photopigment (Goldsmith, 1990). Consequently, most modern placental mammals only have two of the three cone opsins commonly found in birds and reptiles. Primates belong to this line of mammals, but have developed a third opsin of its own. However, the wavelength response profile of the "new" primate opsin does not shift far away enough from those of the existing opsins to greatly improve the overall spectrum sensitivity. Thus, humans have better color vision than most other placental mammals, especially in terms of perceiving shorter wavelength hues, but our color perception is undoubtedly inferior to those of most birds and reptiles.

The selective pressure for various eye forms to evolve may not have existed until the late Vendian and lower Cambrian period, when the phyletic explosion laid the foundation for the evolution of all future animal species. All present day phyla can trace their origin to those 20 or so million years (Morris, 1994, Philippe et al., 1994). From then on, depending on the habitat in which a given species became specialized, various strategies appear to have been adopted to make the light-sensing organ better suited for its task. These structural specializations, such as lens and photoreceptor array formation, constituted the beginning of the formation of true eyes. These functional structures improved light detection and allowed for various degrees of processing of the visual information before it is carried to the central nervous system (CNS). The adoption of fundamentally different visual strategies occurred as the ancestral forms of modern phyla evolved apart.

There are some central differences between the photoreceptors of protostomes (annelids, arthropods, mollusks) and deuterostomes (echinoderms, chordates). The protostome receptor membranes are mostly composed of microvilli (rhabdom), whereas those of deuterostomes contain plates or discs that are typically derived from folded ciliary membranes. Functionally, the protostome receptors depolarize to light reception with the opening of $\mathrm{Na}^{+}$channels; while those of deuterostomes hyperpolarize to light reception with the closing of the same channels. A rare class of protostome photoreceptors hyperpolarize to light but does so by increasing $\mathrm{K}^{+}$conductance rather than by limiting $\mathrm{Na}^{+}$influx (Gorman et al., 1971).

Since eye divergence probably occurred in parallel with phyletic divergence, it is tempting to define eye types along phyletic lines. However, this would be overly simplistic. The presence of a given visual strategy is not only the manifestation of the organism's phyletic lineage, but also a reflection of the inherited genetic history of its predecessors shaped by changing environments and niches. If a particular form of eye is the dominant form in a phylum, it is likely that for most organisms belonging to this phylum, this particular form of eye has been historically well-suited for their survival 
needs. At the same time, evidence shows that similar appearing eye structures could have evolved independently. For instance, the rhabdomeric photoreceptors of the invertebrates are estimated to have arisen as many as 65 times during evolution (von Salvini-Plawen, 1982). Other examples of convergent evolution and re-invention can be found in the similarity of enzymatic derivatives within the lens of both the vertebrates and the mollusks (Tomarev et al., 1993), and the morphological resemblance between vertebrate photoreceptors that have clearly distinct origins (Walls, 1942; Land and Fernald, 1992). The distinctions between visual organ types are particularly blurry among primitive organisms, many of which have maintained the simplicity and diversity of their ancestral forms (Gorman et al., 1971; von Salvini-Plawen and Mayr, 1977).

Long before molecular biology became an area of investigation, nearly two centuries of detailed anatomical and physiological examination of the many forms of eyes have resulted in the formulation of a coherent body of theories concerning the origin of the eye and its development. These works have withstood the test of time and continue to provide valuable references today (Froriep, 1906; Walls, 1942). As molecular methods became more practical and popular over the last two decades, the study of eye development gradually progressed beyond morphological descriptions and began to involve more cytological and genetic manipulations. In broad terms, the understanding of evolution has both benefited from and contributed to the knowledge pertaining to the molecular regulation of development. As will be discussed in more detail in later sections, the continual discovery of "surprisingly" homologous genes expressed during specific periods of invertebrate and vertebrate development is increasingly pointing to a set of comparable mechanisms by which development, including that of the eye, is regulated within many species of the animal kingdom.

The central nervous system, thanks to its highly organized anatomy, is well suited for the study of organ development. The eye is a direct extension of the brain and its anatomical organization directly reflects its developmental lineage. The eye is also very accessible, making it an attractive medium for experimental manipulations. Consequently, numerous studies have been conducted over the years that sought to address questions concerning eye development, in terms of both morphological changes and the genetic regulation of such changes. This is especially the case in Drosophila, where the extensively studied genome has enabled the characterization of numerous molecular mechanisms underlying cellular and morphological development. The discovery of genes important for eye development in Drosophila has in turn contributed to the elucidation of molecular mechanisms controlling eye development in other species, and occasionally vice versa. With the identification and characterization of each new gene and every new 
regulatory pathway, an increasingly better-documented understanding of eye development has begun to emerge. Conceivably, we will eventually be able to not only attribute every aspect of eye development to a clearly defined set of genes and their interactions, but also describe their relationship to homologous genes and gene interactions in other organisms, and perhaps propose a theory with regard to their respective origins.

In the following section, a survey of the known eye forms is presented. Examples are given to underscore the probable role of evolution in shaping the various structures of the eye.

\subsubsection{The many forms of eyes.}

\subsubsection{Simple eyes.}

\section{Pit eye (ocellus)}

This is the most rudimentary form of light-detecting organ, which is only a small step beyond a loose network of photoreceptors in the integument (as is the case in earthworms). It could have emerged and re-emerged independently numerous times in a majority of metazoan phyla. Today, it is found in the most primitive representatives of these phyletic lineages, such as certain hydromedusae and bivalves. In other instances, its presence is supplementary to that of principle eyes, as is the case in some species of insects, where three ocelli are found between the compound eyes in the adult. These accessory ocelli are used for vertical axis orientation during flight, which does not require image resolution (Barnes, 1987). Their development is under a separate genetic regulatory pathway from that of the principle eyes, suggesting separate evolutionary origins.

A single ocellus consists of a group of no more than 100 photoreceptors arranged in a pit within the integument about $100 \mu \mathrm{m}$ in diameter. Depending on the organism, the pit eyes can be of either the "everse" (photoreceptors directed towards the light, as in the insect-mollusk eye) or "inverse" (receptors outer segment directed away from the light, as in the chordate-vertebrate eye) configuration. The photoreceptors are most often derived from ciliated ectodermal cells, but there are instances where they are of non-ciliated ganglion cell origin (Burr, in Ali, 1984). Pit eyes are not capable of forming an image on their receptive field, although there may be refractile structures or reflective linings present in them which improve light perception; and the orientation of the photoreceptors within the pit may in some cases reflect an adaptation to improve motion detection. These additional features represent bridges to more complex forms of eyes. 


\section{Pinhole eye}

This form of eye is a natural step beyond the pit eyes in the course of simple eye evolution. It allows the projection of a visual image onto the receptive field. It consists of a larger "retina" cusp which terminates into a small anterior aperture. As a result, light rays entering the eye are somewhat more parallel, which permits the resolution of an image. The contractile pinhole aperture is functionally the forerunner of the iris, providing some degree of accommodation for light intensity and allowing limited focusing. However, in the absence of an integral lens, the pinhole eye is a necessarily poor-performing eye, as there is an inverse relationship between the resolution and the brightness of the image projected through the pinhole. The only true living example of such eye form is found in the cephalopod mollusk Nautilus, whose eyes are a curious example of "almost perfect" design (Muntz and Raj, 1984), in that the lack of a lens has kept the otherwise sophisticated photoreceptor arrangement in the Nautilus eye from performing anywhere close to their theoretical potential.

\section{Eye with simple lens}

Both the vertebrate eye and cephalopod mollusk eye fall into this group, even though each of them has arrived at its present form from completely different evolutionary paths. The most significant improvement these eyes have over their presumed pinhole predecessors is the inclusion of a refractive lens, which permits the projection of a focused image onto the receptor layer in the retina regardless of the size of the pupil. The shape of the lens is dictated by the environment within which the organism lives. In aquatic animals (fish, gastropods, and annelids), in which such eye forms first emerged, the lens is essentially spherical. Whereas in the land animals, the lens tends to be elliptical, and much of the light-focusing is achieved through cornea (see below). To compensate for spherical aberration outside the central area of the lens, the more advanced forms of eyes have developed a lens containing a refractive gradient; the crystallin density in the lens decreases towards the periphery, thereby lowering the refractive index (Axelrod et al., 1988). The benefits are two fold: 1) the focal length is significantly shortened; 2) the image resolution is improved over a much wider area of the retina. The selective value of this type of lens is attested by its independent occurrences among many taxa, including in the compound eyes of numerous invertebrate species (Land, 1981). By some estimation, as few as 364,000 generations are sufficient for the evolution of such type of eyes from a simple sheet of photoreceptors (Nilsson and Pelger, 1994). 


\section{Corneal eye}

The vast majority of corneal eyes are terrestrial adaptations of the aquatic lenscontaining eye. As land-animals emerged through amphibious transition, the air/corneal interface gradually replaced the lens as the main refractive structure (which in the case of humans accounts for $2 / 3$ of the optic power). The lens itself is given a secondary role as a focusing element. All vertebrates that live on land at least during part of their life cycle or whose evolutionary origin can be traced to land-dwelling forms possess corneal eyes. In another notable example of convergent evolution, the principal eyes of the spider also rely heavily on corneal refraction for image resolution, and can be considered a bona fide member of this group (Land, 1969).

Due to the juxtaposition of the cornea and the lens in the light path, the curvature and constitution of both structures are subject to a variety of adaptations that result in a compromise between sharp vision and "livability", in response to environmental demands. For instance, the rat cornea is nearly spherical, which provides all-around (but mediocre) vision, its lens is overcorrected for spherical aberration as a compensatory measure (Chaudhuri et al., 1983). In animals that lead amphibious lives (shore mammals, diving birds), various strategies are used to achieve optical efficiency in both the aquatic and terrestrial environments. The seals have adopted a flat cornea, relegating the task of refractive compensation to the lens (a throwback to the fish-like eyes); while diving birds possess a lens with powerful accommodation capabilities. During a dive, strong ciliary muscles compress the lens around the equator, such that the lens becomes nearly spherical and protrudes through the rigid iris, thus changing the curvature of the cornea (Sivak et $a l ., 1985)$. In this configuration, the avian eye also takes on a very fish-like appearance. Other than the insect compound eye, the corneal eye has been the subject of most studies on eye development and function, due in large measure to the presence of human eyes within this sub group.

\section{Multiple lens eye}

This rare form of eye occurs in only a few aquatic invertebrates. They represent an alternative solution to the need for a heterogeneous lens. In these eyes, spherical aberration is corrected via successive aspheric surfaces, much like the effect of having multiple lens elements in a photographic camera. In the copepod Copilia, the lenses are arranged in such a way that a front "objective" element projects onto a movable "eyepiece"-receptor element, producing a telescopic effect. The field of view is exceedingly narrow in such an arrangement. However, the movement of the "eyepiece" element is apparently involved in the "point-type" scanning of the visual field, in much 
the same way a spot light scans a theatrical stage (Land, in Ali, 1984). This type of eye, while rare and curious, does illustrate the surprising flexibility that exists in the possible organization of the visual organ.

\section{Concave reflector eye}

This form of eye represents another possible descendant of the primitive pit eyes. Concave reflector eyes are found in some worms, copepods, and at the edge of the scallop mantle. They are characterized by the linear array arrangement of multiple eyes and the presence of a multi-layered concave mirror behind the photoreceptors. The mirror was once thought to help light-gathering only, but more careful analysis revealed that it possesses the geometric quality of the principal reflector in a compound telescope, and is indeed capable of image resolution in collaboration with a Cartesian oval-shaped lens (Dawkins, 1996). Functionally, the linear arrangement of these eyes and the presence of mirrors are similar in principle to the reflective superposition compound eyes in arthropods (see below), and are thus well suited for motion detection.

\subsubsection{Compound eyes.}

\section{Apposition eye}

This is the best known type of compound eye. It is found in diurnal insects and many crustaceans. It may represent the "ancestral" type of all compound eyes.

Each apposition eye is composed of a repetitive array of identical single eye units, or ommatidia. Each ommatidium has a lens, which focuses light on the apical region of the rhabdom - a photopigment-containing structure formed by the fusion of apical membranes (rhabdomeres) of a highly stereotypical aggregation of photoreceptor cells. In contrast to the ciliary type receptors whose photopigments are contained in membranes derived from cilia, rhabdomeric structures are usually derived from microvilli. However, both the rhabdomeric and ciliary photoreceptors are of epidermal rather than deep neuronal origin (of the diverticular type found in the pineal "eye", whose function is related to circadian rhythm regulation rather than vision) and are therefore developmentally homologous with one another. Indeed, microvilli-derived photoreceptors are also seen in primitive chordates (Gorman et al., 1971). Like simple eyes, apposition eyes could have occurred independently numerous times within various invertebrate phyla (von Salvini-Plawen and Mayr, 1977) and may represent the culmination of several convergent evolutionary paths. 
The principal function of apposition eyes, and indeed of all compound eyes, is motion detection. The physical constraints dictated by the receptor unit arrangement are such that to achieve vertebrate-like image resolution, it would require the assemblage of an enormous number of ommatidia spread over an unrealistically large spherical surface (Land, 1981). Several hundred thousand axons per optic nerve is not unusual for an average-sized vertebrate eye; whereas to find more than 10,000 axons in a compound eye optic nerve would be quite exceptional. Even if size limitation is not a factor, the vertebrate eye is still capable of resolving several orders of magnitude better per unit of area than the best of compound eyes, thanks in part to the stratified organization of the vertebrate retina, which allows more neurons to be dedicated to the processing of visual signals before they exit the eye, and in part to generally superior optics (i.e., a single large lens coupled with an adjustable pupil provides better image resolution than multiple lenses without pupil). That said, each ommatidium in a compound eye is still capable of projecting an image, however poorly resolved it may be, onto the rhabdom via sometimes quite sophisticated optical tools. Just like its simple eye counterpart, the terrestrial insect compound eye depends on its corneal surface for image formation. Also, some ommatidial lenses have variable refractive indices that improve their performance, much like the lens of many simple eyes. There are indications that in spite of the major physical differences between the vertebrate and invertebrate lenses, there is some homology in the genetic regulation of their development, owing perhaps to the similar strategies by which lens proteins are recruited (Tomarev and Piatigorsky, 1996).

\section{Afocal apposition eye}

The lens element in this type of eye projects an elongated beam, rather than a focused spot on the rhabdom. It is the consequence of a very short focus length. This arrangement is believed to be an intermediate form between apposition eyes and superposition eyes. Or rather, its existence made the emergence of superposition eyes possible. Eyes of this type occur in butterflies and several other insect classes.

\section{Neural superposition eye}

The term "superposition" refers to the fact that multiple ommatitia are involved in resolving the same detail of an image, in contrast to the functionally independent ommatidia of an "apposition" eye.

The neural superposition eye is characterized by the differential orientation of individual - non-fused - rhabdomeres within each ommatidium. Namely, all rhabdomeres occupying homologous positions in adjacent ommatidia face the same direction, which is 
at a slight angle with rhabdomeres occupying other positions of the ommatidium. This arrangement presented a challenge to investigators at the turn of the century who wished to understand how any useful information could be extracted from such an awkward receptor array. Interesting (and later proven to be correct) hypotheses were proposed to account for the unusual anatomy of such eyes (Vigier, 1908), but it was not until the late 1960s that the underlying neuronal wiring was elucidated. Once the functional organization was explained, the awkwardness of the anatomy disappeared. The rhabdomeres and ommatidia in the neural superposition eye are positioned in such a way that eccentric rhabdomeres are oriented in the same axis as the central rhabdomeres in the adjacent ommatidia. These similarly oriented rhabdomeres project to the same processing area within the lamina (the insect equivalent of the visual cortex or tectum), effectively making the strength of visual signals reaching the lamina proportional to the number of rhabdomeres in the orientational pseudo-cluster that are stimulated by the visual cue (Kirschfeld, 1967). Evidently, the anatomy of the neural superposition eye is an adaptation for better motion detection, which, as is generally the case in the compound eye, comes at the expense of image resolution. In a way, eyes of this type may be thought of as apposition eyes with overlapping ommatidia. The most interesting aspect of these eyes from a developmental perspective is perhaps the routing and mapping of the visual axons. Not only do these axons have to find their appropriate target areas in the lamina (corresponding to the ommatidia from which they originate), but they also have to make connections according to the orientation of the rhabdomeres. If we can understand the guidance mechanisms responsible for the wiring of such a network, we can probably go a long way towards learning how the wiring of our own visual pathways is accomplished.

\section{Refracting superposition eye}

This type of eye differs from apposition eyes mainly in the shape of the lens. It is believed to have evolved in nocturnal insects such as the moth, presumably as a means to improve light sensitivity. In essence, the lens in these eyes is cylindrical rather than flat. The cylinder acts as a non-magnifying "telescope" by having the focal point in the middle of the cylinder, effectively rendering the apical and basal surfaces of the lens cylinder into separate optical elements, with the basal surface projecting a parallel output beam. The wall of the ommatidium is transparent just above the rhabdom, allowing light from the neighboring ommatidium to reach the rhabdom as long as it occurs at a certain angle. The end result is similar to that achieved by neural superposition eyes. By some estimates, such an arrangement increases light sensitivity by up to one order of magnitude over comparable apposition eyes (Land, 1984). Such eyes are found in nocturnal insects. 


\section{Reflecting superposition eye}

Decapod crustaceans are the sole possessors of this type of eye. It represents a different solution to the same problem solved by refractive superposition eyes. Instead of relying on the telescopic quality of refractive lens cylinder however, the reflective eye uses a right-angled mirror box array to direct light towards the rhabdom. Due to the right angle orientation and the length of the mirror structure, the only requirement for the incident light rays to be directed towards the rhabdom is that they occur at an angle to one of the mirrors. Thus, oblique light rays which might otherwise miss the rhabdom are captured by the receptors through relatively uncomplicated mirror geometry. In spite of its "awkward" appearance, the mirror arrangement seems very well adapted for this function. In the crayfish, there is even a weak local corneal lens whose role may be to help direct stray light onto the mirror reflectors (Bryceson, 1981).

\section{Parabolic superposition eye}

This form of eye contains elements of both apposition and superposition eyes (Nilsson, 1988). It has a corneal lens, and a reflective lining along the cone wall leading to the rhabdom. Depending on the angle of incidence, the light can take quite different optic paths on its way to the rhabdom, in that the mirror and lens arrangement can give both apposition and superposition attributes to the eye. This type of compound eyes may represent a intermediary form between refracting and reflecting superposition eyes. It has been encountered in brachyuran and anomuran crabs only.

\subsection{Eye development.}

2.3.1 The sequence of events during eye development.

To understand eye development is to understand its regulation. In order to effectively study the regulation of eye development, we need to first recognize the anatomical events which define eye development. Currently, much has been learned about the sequence of events leading to the formation of two types of eyes: the insect compound eye, specifically that of the dipteran fruitfly Drosophila melanogaster, and the vertebrate eyes (mammalian, chick, amphibian, fish; which develop similarly). The compound eye and vertebrate eye develop according to very different plans from different tissues, yet there are intriguing instances of commonality and similarity in terms of how the respective tissues behave during development, as well as the nature of the genetic 
regulation of both developmental pathways. Before addressing the molecular aspects of eye development, a brief overview of the anatomy of the developing Drosophila and vertebrate eyes may be necessary.

\subsubsection{The genesis of the Drosophila eye.}

During development, one of the fundamental differences between the insect and the vertebrate is that the former goes through a syncytial blastoderm stage, where cellular fate determination occurs via intracellular mechanisms (for review, see Cagan, 1993; Venkatesh, 1993). In Drosophila, six cells of the syncytial blastoderm are fated to become the eye disc and grow as a monolayer. At the end of larval life, the cells in the eye disc stop dividing and begin the process of differentiation, which occurs in a synchronous and sequential fashion in populations of cells along the anterior-posterior axis. The result is a groove forming in the epithelium known as the morphogenetic furrow. The cells posterior to and away from the groove are developmentally older than those anterior to and in contact with the progressing furrow. This characteristic makes it possible to "look into time" on an experimental preparation of the imaginal eye disc. The differentiating ommatidial precursor cells occur in clusters of six to seven cells, of which the photoreceptor R8 is the first to emerge, followed by the diametrically positioned pair R2 and R5, then R3 and R4. The next wave of differentiation adds R1 and R6, then finally R7 to the cluster, completing the formation of the 8 cell photoreceptor rosette in each ommatidium. Four cone cells are added around each receptor rosette during the third larval instar. These cells give rise to the lens of the ommatidium and are not photoreceptors (not to be confused with vertebrate cones). The rest of the cells in the mature ommatidium, six pigment cells and four bristle mechanoceptors, are added during pupation. The rhabdomeres of R1 through R6 are arranged in an asymmetrical trapezoid and constitute the full thickness of the retina, whereas R7 and R8 rhabdomeres are smaller and shorter and take on the same axial position, with R7 rhabdomere occupying the apical segment, and R8 rhabdomere below it. There are between 750 to 800 ommatidia per typical Drosophila eye.

Axon projections to the first optic ganglion (lamina) occurs during the third larval instar immediately following the differentiation of all receptor cell types. The eight axons from the same ommatidium are bundled together in a fascicle during pathfinding. The axons of R1 through R6 make precise stereotypical connections with their target positions on the medial border of the developing lamina after exiting the optic stalk, while the axons of R7 and R8 continue on to make retinotopic connections in the developing 
second optic ganglion (medulla). The R7 and R8 maps in the medulla are aligned but are arranged in distinct layers. Recent evidence shows that the pathfinding process is likely guided by positional cues present along the optic tract (chemo-affinity) and within the target areas of the ganglia. Once reaching the destination in the ganglia, cell-cell interactions between adjacent axons establish their mutual boundaries (Kunes et al., 1993). The pathfinding mechanism appears quite robust, as mutations which grossly affect the development of particular receptor cell types have no noticeable effect on the overall connection pattern of unaffected cells. Ashley and Katz (1994) have reported that axons from a mosaic eye, in which ommatidia over-expressing the R7 receptor are surrounded by ommatidia that lack R7, can still map to appropriate positions in a largely empty medulla. These axons may project collaterals into vacant termination sites, but only if two or more axons associated with over-expressed R7 receptors from a single ommatidium attempt to innervate the same termination site. These findings suggest that boundary establishment in the ganglia is an inherent function of the axons themselves, possibly via competition. Evidence also indicates that photoreceptor axon innervation is part of the triggering mechanism controlling the differentiation of lamina precursor cells; thus the development of the sensory and CNS components of the Drosophila visual system appear to be partially coordinated in such manner (Selleck and Steller, 1991).

\subsubsection{The developing vertebrate eye.}

The vertebrate eye owes its inverted retinal arrangement (photoreceptors facing inward) to its developmental origin. Vertebrate photoreceptors are derived from the ciliated ependymal cells of the neural tube. Given that the ciliated surface lines the tube cavity, and the outer segments of photoreceptors are homologous to the cilia, the inverted arrangement is the natural outcome of the process by which the vertebrate eye is formed. Conceivably, an everse retina could have evolved if the photoreceptors had been derived from the surface ectoderm (as in the flies), or if the retinal precursors migrated out of the neural fold before neural tube closure. However, there is no indication that either scenario occurred in the ancestral vertebrates.

During neurulation, the precursors of the optic organ are noticeable as large columnar cells at the lateral folds of the neural groove. After the closure of the neural tube and the formation of the rudimentary forebrain ventricle, the optic vesicles gradually extend laterally from the forebrain as out-pockets (Fig. 2.1). The interaction between the optic vesicle and the superposing surface ectoderm triggers the development of the lens 

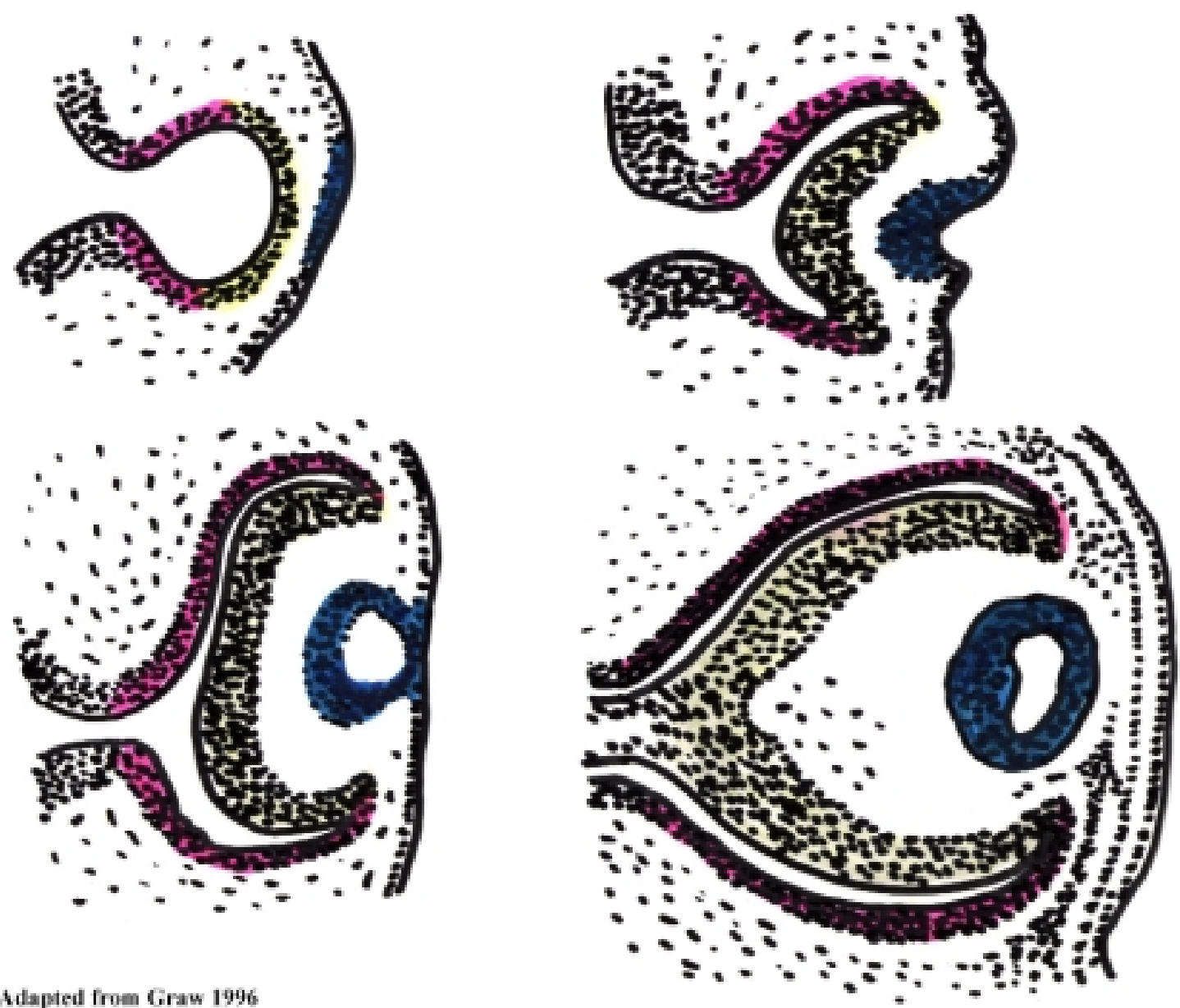

Adapted from Graw 1996

Figure 2. 1 The developing vertebrate eye. The blue shaded area denotes lens placode and subsequent lens structures. Yellow shaded area represents neural retina tissue. Red shaded area represents pigmented epithelium tissue.

vesicle, which begins with cell thickening within the lens placode and its subsequent invagination, and terminates with the fully formed lens vesicle "budding off" the development of the lens from the epithelium [Lens development was the subject of a pioneering study on organ genesis by Spemann a century ago, where he introduced the concept of induction; although the interpretation of his results was later found to be inadequate (Oliver and Gruss, 1997)]. The invagination of the lens vesicle occurs in concert with the collapse of the optic vesicle; the latter eventually forms a bilayered cusp with a narrowing furrow along its ventral axis continuing into the optic stalk. The furrow then fuses to become the embryonic fissure (or optic fissure) just as retinoblasts begin to differentiate. The two layers of the collapsed optic cup give rise to the retina through rapid cell proliferation and differentiation. The outer layer becomes the pigmented epithelium (PE, or pigmented retina). The inner layer becomes the neural retina, which gradually 
loses its pseudostratified cellular conformation as it produces successive classes of retinal neurons, most of which undergo cell division at the outer margin and migrate inward as they mature (i.e. from the PE towards the vitreous) and eventually form anatomically and functionally distinct neuronal layers. Cellular differentiation begins at the posterior pole of the optic cup and spreads towards the periphery. If analogy is to be made with the Drosophila morphogenetic furrow, one might picture that in the vertebrate eye, there are successive concentric morphogenetic "furrows" progressing from the posterior pole towards the rim of the optic cup as cells differentiate. According to Sidman (1961), the retinal ganglion cells are born first, their growing axons extend towards the future optic disc along the surface of the retina and would eventually become fasciculated as they emerge out of the optic cup to form the optic nerve. The birth order of other retinal neurons tend to follow their positions within the retina, i.e., cells of the inner layer are born before cells of the outer layer. However, there is much overlap in the differentiation sequence, as some amacrine cells and photoreceptors, whose terminal positions are in the outer aspect of the inner nuclear layer and the outer nuclear layer, respectively, have clearly stopped dividing prior to the bulk of bipolar and horizontal cells are born, even though the latter occupy more inner positions. The differentiation process continues over a protracted period of time, and does not reach completion until well after birth in many species. The Müller cells are the last cell type to emerge. They eventually form the supportive scaffold of the neural retina.

As the neural retina differentiates, the lens matures through successive deposition of elongated lens fibers, it also induces the formation of the anterior chamber in the adjacent mesodermal tissue. The neurocrest-derived mesenchymal tissues anterior to the chamber eventually form the inner part of the cornea; whereas the same tissue in contact with the developing lens would give rise to stroma of the iris, the only ocular muscle that is not of mesodermal origin (Graw, 1996).

The circulatory system in the vertebrate eye varies widely depending on numerous factors. Namely, the size of the eye, the lifestyle of the animal, and environmental conditions, can all have significant effects on the organization of the system. In general, large eyes or highly active animals tend to have significant vascularization within the retina, whereas slower animals and/or smaller eyes tend to have less (Walls, 1942).

The projection of retinal axons in the CNS also varies significantly between vertebrate species. For instance, all retinal axons decussate in the bird; whereas in primates, nearly half of the axons remain ipsilateral (Walls, 1942). Regardless of the axon projection scheme however, the visual areas of the CNS are always highly organized and retinotopically mapped. Thus, from insects to man, the topographic association between the eye and the CNS appears to be a necessary component of the visual system. It would 
be intriguing, but not surprising, if conserved molecular mechanisms are found to regulate visual axon guidance within the developing CNS of these different organisms (Holt and Harris, 1993). For instance, the nematode axon guidance molecule UNC-6 and its receptor UNC-5 have been shown to have mammalian counterparts (netrin) that perform quite similar functions (Goodman, 1994). Netrin is suspected to play a role in guiding the exit of ganglion cell axons at the optic nerve head (Otteson et al., 1998). As more molecules are identified and characterized as having a role in visual axon guidance, we will undoubtedly uncover additional homologies between invertebrates and vertebrates.

\subsubsection{The genetic control of eye development.}

The description in the preceding section shows that in spite of divergent evolutionary paths and radically heterologous body plans, there are noticeable parallels between the developing Drosophila and vertebrate eyes. For instance, the sequential order of receptor differentiation in both types of eyes may be the consequence of similar inductive events. To understand how complex structural forms such as the insect and vertebrate eyes could have evolved from simpler ancestral forms, one has to look at the very source of evolutionary changes, i.e., the genes which regulate development. The extra energy required to create new or more complex structures would be tolerated by natural selection only if the organism's overall fitness is not compromised by the process. Given the importance of vision (or mere photosensitivity) to the survival of most metazoan animals, the pressure against rapid changes in the genes controlling the development of essential structures of the eye must be substantial.

Suppose that the inverted vertebrate retina can be made more efficient by its eversion, and that vertebrates have retained the genetic capability to grow eyes the same way invertebrates do - from the integument, in much the same way that chicks have retained the genes to grow teeth (Kollar and Fisher, 1980). What conditions must be met for the transformation to occur? The vertebrate eye is already highly evolved and functionally well appointed. A fundamental rearrangement of the retina would have no selective value unless the existing morphogenetic programs are first disabled. Such changes could occur if the organism becomes adapted to living in a lightless environment, such as underground or in deep ocean, where the loss of eyes through genetic mutations may be tolerated. Walls (1942) argued that the ancestral placental mammals and snakes have undergone just such fossorial existence during their respective evolutionary histories, which resulted in the loss of several types of photoreceptors, and in the case of the snakes, numerous ocular structures. However, neither group maintained that lifestyle 
long enough or thorough enough to lose their eyes. However, re-arrangement of the eye does appear to have gone quite far in some deep-sea invertebrates, an example of which is the crustacean Ampelisca, in which the compound eye has taken on the appearance of a lense-containing simple eye (Dawkins, 1996).

Nevertheless, within the basic constraints imposed by the existing developmental programs, many less dramatic changes can take place in the eye and affect visual performance. For instance, genetic drift can lead to the emergence and adoption of different lens geometries, in response to shifts in environmental conditions.

In practice, how do these changes take place at the molecular level? Wagner (1994) proposed that selective pressure of evolution acts not so much on the genes involved in the generative aspects of development, but rather on those genes which maintain the structures once they are formed (morphostatic). These genetic mechanisms constitute the "unspectacular" side of development that has been somewhat overlooked by investigators in the past. The rules for the maintenance of structural integrity are by nature more plastic than those which regulate the emergence of structures, as deviation from a set morphogenetic pathway is likely to lead to dysmorphogenesis. Changes to genes which control structural integrity are therefore more likely to be tolerated compared to alterations to generative genes. In time however, enough changes could be retained through the morphostatic genes to create a permissive environment for the emergence of structural features that have selective advantages.

Alternatively, the genetic changes could occur through a "safe" mechanism - gene duplication - where the presence of the old gene guarantees that the original function is not compromised, while the duplicate gene(s) is(are) free to explore alternative roles that may ultimately prove beneficial to the organism. This mechanism was first proposed by Ohno in 1970; it has since been shown to occur quite frequently in the vertebrate genome (Ruddle et al., 1994a,b; Holland et al., 1994).

A hypothesis has also been proposed to account for the strict conservation of regulatory genes in all organisms (Duboule, 1994). It is based on the assumption that timing enjoys a critical importance during morphogenesis, where once the permissive "time window" for a structure to develop has elapsed, there are no means for going back (Duboule coined it the 'Einbahnstraße', or 'one way street'). Hence, even slight modifications to genes that can shift the timing of their expression (heterochrony) during organogenesis could have profound effects on the overall development. These effects are seldom beneficial to the host organism. Thus, developmental events outside such critical time windows may be flexible enough to allow quite a lot of divergence to occur during evolution; but the period of major cellular differentiation and vital organ induction is 
typically a gated Einbahnstraße, where one is likely to find many conserved regulatory genes at the gates.

Evidence shows that many highly conserved genes are indeed expressed during periods of organogenesis that can be characterized as Einbahnstraßen, such as the genes that mark the boundaries of differentiating structures, or those controlling the timing of cellular differentiation. Moreover, some of these genes are frequently duplicated in the genome of higher organisms, for instance the genes belonging to the homeodomaincontaining superfamily, which includes such notable sub-families as Hox, Pax, MADS, etc. (Manak and Scott, 1994). The discovery of Hox was made in the Drosophila nearly two decades ago, which paved the way for much of the break-throughs in the understanding of the genetic regulation of development. In higher vertebrates, entire families of these genes may be arranged in repetitive clusters on a chromosome based on their sequential order of expression during body plan determination (Hox genes), or they may be dispersed on different chromosomes and expressed concurrently during development (Pax genes; Chalepakis et al., 1993). Many of the genes have tissue-specific expression patterns, and the specificity is often conserved across taxonomic boundaries. Numerous regulatory functions have been attributed to these genes because they typically code for transcription factors containing one or more DNA binding domains. One of these genes, Pax6, has been found to play several key roles during eye development, which are discussed in Section 2.3.3.1, and provided the first startling evidence that homologous molecules regulate key events during the development of very different eyes.

The fundamental questions relating to evolution and development are being addressed through comparative genetic studies (Wolpert, 1994a,b). Significant insights into organ evolution may be gained by examining molecular regulation of homologous organ development in different species. For well over a decade, a great deal of research has been conducted to obtain a clearer view of the genetic regulation of Drosophila eye development. Often, developmentally significant genes discovered in the Drosophila have led to the identification of vertebrate homologues, many of which are expressed in equivalent tissues and have surprisingly similar functions (Reh and Cagan, 1994; Oliver and Gruss, 1997; Bulfone et al., 1998). 


\subsubsection{The eye genes.}

An all-inclusive survey of what is known about the genetic regulation of eye development is fast becoming impractical, in view of the exponential rate at which the field has expanded in recent years (for reviews, see Cagan, 1993; Venkatesh, 1993; Graw, 1996; Freund et al., 1996). However, it is possible to focus on a limited number genes and genetic pathways, which have been experimentally shown to represent certain classes of genes and types of interactions, and gain through them a glimpse of the complex organization of the molecular regulation of eye development (Halder et al., 1995; Oliver and Gruss, 1997).

For example, cell-cell interaction plays an important role during both the differentiation of vertebrate retinal neurons and the differentiation of Drosophila photoreceptors. In vitro experiments have shown that mammalian photoreceptor progenitors could differentiate into various classes of retinal neurons if they are part of a rosette-like aggregate with appropriate types of photoreceptors or neurons. Namely, the early progenitor cells are fated to become rods if cultured alone, but when co-cultured with cortical neurons, they tend to take on the attributes of retinal ganglion cells (Rhe, 1992). This is reminiscent of the process by which cell fate is determined in the Drosophila ommatidia. There is mounting evidence that such a process employs signal molecules and receptors that are present in both the fly and the vertebrate (see Section 2.3.3.2).

Conceptually, there are four identifiable steps (albeit seamless in reality) during organogenesis; in each of these steps one might find homologous genes playing similar roles in different organisms (Oliver and Gruss, 1997). First, the progenitor tissue becomes competent to respond to differentiation cues, in a process that does not depend on external signaling molecules but on internal timing mechanisms. Then, the competent tissue progresses towards an irreversibly committed (or biased) state (equivalent to the concept of Einbahnstraße), under the guidance of early patterning genes. After that, additional genes regulate the specification, and finally the differentiation of the biased tissue into its terminal state. The following survey of genes, while far from being comprehensive, contains those that have been shown to play significant roles during the recognized major steps. 


\subsubsection{The early patterning genes.}

\section{Eyeless (ey), Small eye (Sey), Aniridia}

The genetic characterization of the Drosophila eyeless (ey) mutation is perhaps the best known example of how investigations focused on an isolated gene could generate great insight into the developmental pathway in which the gene plays a role.

Ey is a spontaneous semi-dominant mutation in the Drosophila that affects the formation of the compound eye (the ocelli are not affected). The genetic cause of the ey mutation was not understood until quite recently, by way of a related mutation in the mouse - Small eye (Sey). Sey was identified as a model for Aniridia (lack of iris) and Peter's anomaly (anterior chamber and corneal defects) in humans (Glaser et al., 1990; van der Meer-de Jong et al., 1990). Homozygous Sey mutants fail to develop the eye and/or the nasal cavities. Hemizygous mutant embryos have delayed closure of the optic fissure and typically have small eyes. After the Sey gene was isolated, its sequence was found to contain a homeobox and a paired box domain that identified it as Pax6, a gene previously discovered by its sequence homology to other Pax genes (Walther et al., 1991; Walther and Gruss, 1991; Hill et al., 1991). The Drosophila ey gene was subsequently recognized as a homologue of the mouse Pax6 (Quiring et al., 1994; Hanson and van Heyningen, 1995). In the developing Drosophila, the ey gene expression pattern was found to be homologous to that of Pax6 in the mouse. The Drosophila ey amino acid sequence showed $94 \%$ identity with murine Pax6, as well as with Pax6 sequence from human and birds. It had 93\% identity with the zebrafish Pax homologue within the paired domain, and $90 \%$ identity within the homeodomain.

In all species examined, Pax6 expression has been noted in numerous areas of the developing nervous system (Chalepakis et al., 1993), yet only the eye appears to be affected in loss-of-function Pax6 mutants, in the form of disrupted early eye development. Gehring's group demonstrated a few years ago that by targeted expression of complementary ey DNA in various imaginal discs, ectopic compound eyes can be induced in many regions of the Drosophila body, such as the legs, the antennae, and the wings (Halder et al., 1995a). A notable aspect of their findings was that the ectopic ommatidia were essentially normal in their cellular complement and organization. By implication, the majority of the estimated 200 or so genes necessary for Drosophila eye development are downstream of Pax 6 , such that Pax 6 acts as a molecular switch that "turns on" the eye development cascade at the appropriate time and place (Halder et al., 1995b). Or, to place Pax6 within the context of the four phases of organogenesis outlined 
above, Pax6 must be situated at the very beginning of the "bias" phase, when the entire Drosophila integument is still "competent" to respond to eye development signals.

Following these discoveries, Gehring and associates isolated Pax6 homologues in organisms ranging from mollusks, some of which have very organized and almost vertebrate-like eyes, to primitive chordates possessing nothing more than rudimentary ocelli, to various vertebrates with typical lens-containing eyes, and found that regardless of the origin, the Pax6 genes from any of these organisms can induce ectopic eye development in Drosophila, effectively showing that the gene is both structurally and functionally conserved (Callaerts et al., 1997; Glardon et al., 1997; Tomarev et al., 1997; Glardon et al., 1998). In vertebrates, a minimum of 2500 genes are believed to be needed for the formation of the eye, representing 10 times more genes than those required to build a compound eye, yet Pax 6 appears to be "retained" as a regulator of vertebrate eye development with little change in its molecular structure. Notably, Pax6 is principally involved in the induction of vertebrate lens development (Altmann et al., 1997). Is Pax6 a relic from the ancestral eye, from which all eyes descended, or has it been independently recruited during separate evolutionary events?

The evidence showing that Pax6 is conserved between organisms as phylogenetically distant as fruitflies and mammals has led some investigators to reevaluate the dogma regarding the origin of divergent eye forms. Is it possible that the compound eyes of the insects, the vertebrate-look-alike everse eyes of the cephalopods, and the lens-containing inverse eyes of the vertebrates all derived from a common ancestral eye? In view of the Drosophila data, it seems logical to characterize Pax6 as a "master gene" of eye development, one that is at the beginning of the "eye genes" cascade. However, it is also possible to characterize Pax6 as a more generic molecule, whose existence likely predated that of any bona fide visual organ, and whose function is to regulate the timely expression of genes that later became important for the formation of the visual organ, such as the genes regulating the differentiation of photoreceptors and, perhaps still later along the evolutionary timeline, those specifying the synthesis of lensrelated proteins. As such, Pax6 could have been independently recruited or retained several times for these purposes during the evolution of various eyes. The hypothetical ancestral eyes in which $\operatorname{Pax} 6$ took on the role of a key regulator of organogenesis may have been little more than photoreceptor aggregates in the integument and/or directly within the nervous tissues (the vertebrate eye being derived from the latter). In fact, one of the characteristics distinguishing Pax6 from other members of the Pax gene family is that it is constitutively expressed before and during the formation of much of the developing nervous system, with no indication of segmentary restrictions (Walther and 
Gruss, 1991; Callaerts et al., 1997), suggesting that it once had, and probably still has, other roles in the developing CNS. Shortly after Pax6 was identified as an important regulator of eye development, a Pax6 homologue, $v a b-3$, was isolated in the naturally eyeless nematode $C$. elegans; $v a b-3$ has been shown to play an important role in specifying tissue fate within selected regions of the developing head (Chisholm et al., 1995). Recent evidence also suggests that Pax6, in association with other genes, may be involved in regulating the development of non-nervous organs, such as the pancreas (Habener and Stoffers, 1998), and in the maintenance of certain cell types in mature tissues, namely amacrine cells in the retina, and cells in the lens and cornea (MacDonald and Wilson, 1996).

Evolution of the metazoan eyes could have begun with the expression of genes coding for photoreceptors in the head region, in close association with the CNS. A Pax6/vab-3 -like molecule, which controlled head patterning, was a natural candidate for initiating the formation of receptor clusters, regardless of whether they were on the integument or in the linings of the primitive nervous system (these organisms, like many of their counterparts today, were likely small and transparent), thus becoming closely associated with the development of several types of primitive visual organs. Later, as more structural genes were recruited and various complex forms of eyes emerged along diverging phyletic branches, Pax6 was retained as a regulator of the early events during the morphogenesis of the eye, and may even have acquired new functions (e.g., the vertebrate lens development). Consequently, even as thoroughly different forms of eyes emerged during the course of evolution, their developmental Einbahnstraßen continued to intersect at various molecular crossing points, one of the earliest of which is gated by Pax6. This is not a unique phenomenon; the same type of gene sharing among heterologous developmental pathways is believed to occur in the regulation of Drosophila wing and vertebrate limb development by genes of the hedgehog family (Fietz et al., 1994), and in the expression of Drosophila Msh-like homeobox genes - Hox 7.1 and Hox 8.1 - in the developing vertebrate optic cup (Monaghan et al., 1991). The molecular and functional conservation of Pax6 among different species may be an example of how genetic homology does not necessarily equate common ancestry at the organ level, but rather suggests that components of such organs could have derived from common origins. Some of the components may even predate the emergence of the organs proper.

While Pax6 clearly plays a critical role in triggering eye development at an early stage (Fig. 2.2), it is neither the only gene nor the earliest one to befit the title of a "master gene". Indeed, another Pax6 like gene, twin of eyeless (Toy) has recently been 

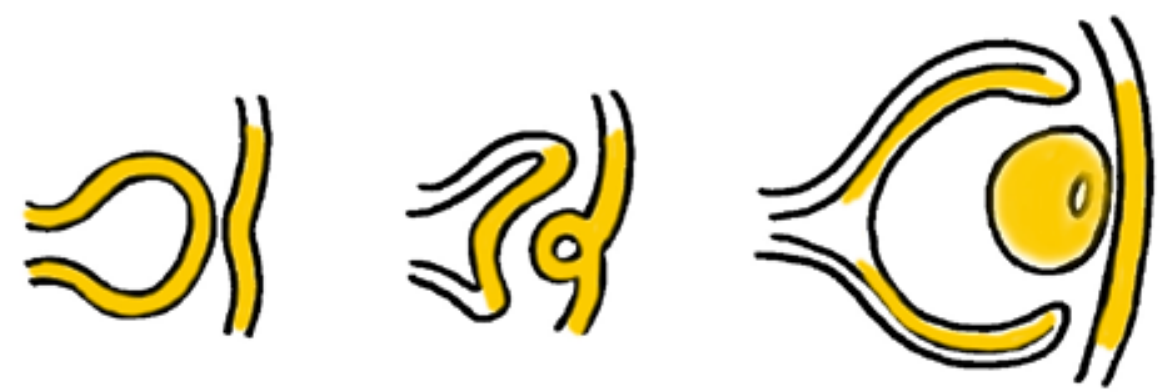

Pax6
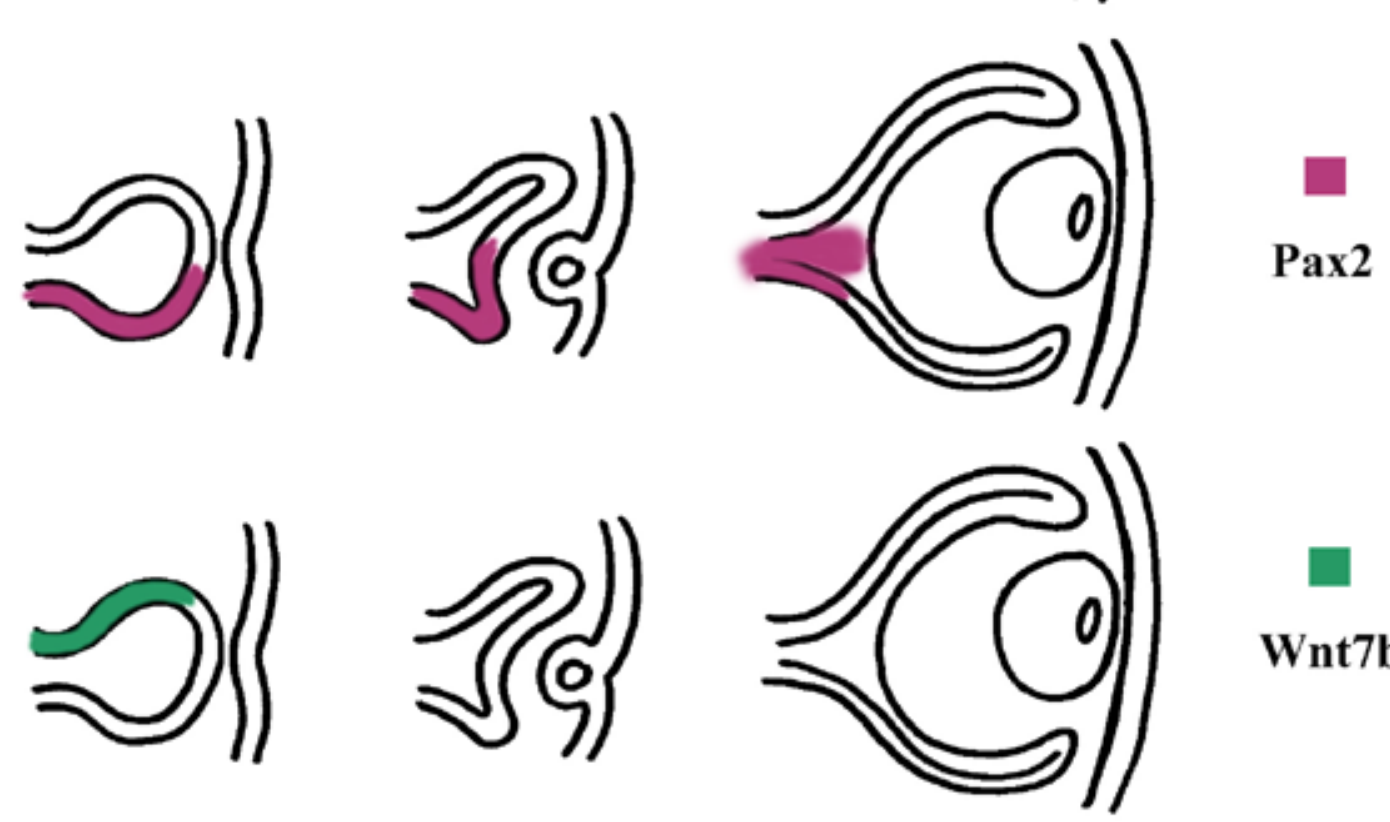

\section{Wnt7b}

Fig. 2.2 The expression patterns of regulatory genes during eye development. The three examples illustrated, Pax6, Pax2, and $W n t 7 b$, are involved in establishing the orientation and boundary of developing retinal structures. Pax6 is involved in specifying tissues which give rise to the retina and the lens. Pax2 is believed to define ventral retina and the optic stalk. Wnt $7 b$ is involved in defining the dorsal aspect of the developing eye. The expression patterns change as the ocular structures become more differentiated. During later stages of development, these genes are believed to play a role in maintaining the differentiated state of specific retinal cell types.

dentified in Drosophila, which appears to regulate ey expression by binding to the latter's enhancer element (Czerny et al., 1999). Interestingly, Toy is a closer molecular homologue of the vertebrate Pax6 gene than $e y$. In vertebrates such as the mouse, optic vesicles can still form in homozygous Sey mutants, and Pax6 expression is not restricted to cells in the anterior neural plate that give rise to the optic vesicles (Grindley et al., 1995). The spectacular growth of ectopic eyes in Drosophila triggered by Pax6 expression has not been replicated in vertebrates. Conceivably, while ey may be at or near the beginning of the genetic cascade regulating the "bias" phase of Drosophila eye development, its homologue Pax6 could occupy a somewhat more downstream position in the vertebrate equivalent. Alternatively, the regulatory cascade controlling the 
early phase of vertebrate eye development may be much more complex and redundant than that of the flies, and thus more difficult to disrupt or manipulate. What is increasingly clear is that Pax6 is one element in an interactive network of molecules involving numerous stimulatory, inhibitory, and feedback pathways.

\section{Pax2, sparkling (spa)}

Another member of the Pax gene family that has been demonstrated to play an important role during vertebrate eye development is $\operatorname{Pax} 2$. Pax2 does not have a homeodomain, which distinguishes it from Pax3, Pax-4, Pax6 and Pax7, and puts it in the same sub-family as $P a x 5$ and $P a x 8$, with which it likely shares common origin through duplication. Zebrafish actually has two Pax2 genes with slightly different expression patterns and functions (Chalepakis et al., 1993; Pfeffer et al., 1998). The expression pattern of $\operatorname{Pax} 2$ during development is wide spread, although it is later than and does not overlap with that of Pax6. In the neural tube, Pax2 is expressed within post-mitotic neuroblasts in the intermediate gray, colocalized with $\operatorname{Pax} 5$ and $\operatorname{Pax} 8$ expressions. It is strongly expressed in the developing metanephric and urogenital tissues. In the head, its expression is localized to the otic vesicle, optic vesicle, and eventually becomes limited to the optic disk and optic stalk/ optic nerve (reviewed in MacDonald and Wilson, 1996; see Chapter 5). Absence of normal Pax2 protein expression, such as in the kidney and retinal defect ( $K r d$ ) mutant mouse (see Chapter 5), leads to a dorsalization of cells in the ventral optic stalk and a delay in fissure fusion, which disrupts optic nerve formation and causes retinal degeneration (Otteson et al., 1998).

While there is no mutagenetic evidence suggesting $\operatorname{Pax} 2$ is involved in the early patterning of the eye Anlage, the complementary nature of its expression pattern with that of Pax6, combined with its role in controlling cellular differentiation in the optic stalk, makes it a strong candidate as a member of regulatory pathways involving other patterning genes. Indeed, the expressions of both Pax6 and Pax2 appear to be partially regulated by the same midline signaling molecules of the Hedgehog family. Namely, Sonic hedgehog $(\mathrm{SHH})$ and Tiggywinkle hedgehog (TWHH) over-expression in zebrafish can lead to ectopic expression of $\operatorname{Pax} 2$ throughout the optic vesicle, and a concurrent reduction in Pax6 expression, resulting in severe malformation of the eyes. Conversely, in the cyclops mutant zebrafish, in which the expression of $\mathrm{SHH}$ and $\mathrm{TWHH}$ is deficient in the rostral forebrain, the opposite occurs: the expression of Pax 2 is reduced and there is ectopic over-expression of Pax6 across the midline, which is believed to cause the fusion of the two retinas (MacDonald et al., 1995), a condition also seen in holoprosencephalic humans as a result of $S H H$ defects (Roessler et al., 1996). 
A Drosophila homologue of Pax2, Pax5 and Pax8, sparkling (spa), has been identified through sequence homology (Fu and Noll., 1997). Spa is expressed in the cone, primary pigment and bristle cells, as well as other parts of the developing nervous system, and may be functionally equivalent to vertebrate $\operatorname{Pax} 2$.

\section{Sine oculis, Six3}

The Drosophila sine oculis is another early expressed homeobox-containing patterning gene, whose expression domain is similar to that of eya. Like eya mutants, sine oculis mutants also exhibit abnormal cell death anterior to the morphogenetic furrow and have disrupted eye development. Moreover, the mutant flies have abnormal optic lobe development, demonstrating that sine oculis is critical for the early development of the entire visual system (Cheyette et al., 1994; Serikaku and O'Tousa, 1994; Halder et al., 1998).

Sine oculis homologues have been identified in mammals, chicks, and zebrafish. In mammals, gene duplication appears to have occurred at least 4 times, several members of the Six gene family are co-expressed with Eya-1 and Eya-2 (Oliver and Gruss, 1997), indicating likely functional homology with sine oculis. One member, Six-3, is expressed in the anterior neural plate, prospective eye field, optic vesicle, optic stalk, the lens and nasal placode, and when expressed ectopically in the fish, is able to induce lens formation in the otic placode (Oliver et al., 1996). Indeed, injection of Six-3 RNA into fish embryos has been shown to trigger retina development in the midbrain and cerebellum via ectopic expression of Pax6, Rx-2 (see below), and endogenous Six-3. (Loosli et al., 1999). Through duplication, the fish (zebrafish) actually has two mammalian Six-3-like genes, Six-3 and Six-6, where Six-6 is functionally closer to mammalian Six-3 (Pignoni and Zipursky, 1997). A chick Six3 homologue, $c$ Six3, has also been identified recently; it is expressed in the same developing tissues and may perform the same functions as its insect and mammalian counterparts (Bovolenta et al., 1998).

Consistent with other patterning genes, the expression of $c \operatorname{Six} 3$ appears to become more restricted as organogenesis progresses. In the retina, its expression is eventually limited to the ganglion cells, in the same way Pax6 expression becomes restricted to ganglion cells and amacrine cells, $C h x-10$ (see below) expression becomes restricted to bipolar cells, and Prox-1 (a homologue of Drosophila prospero that is also implicated in the regulation of lens crystallin synthesis) expression becomes limited to horizontal cells (Belecky-Adams et al., 1997). These increasingly restrictive patterns of expression accompany the emergence of functional layers made up of the differentiated cell types, suggesting that a certain link exists between the differentiation of such cells - controlled 
by the patterning genes - and the segregation of the cellular layers, for which the interaction between the various patterning genes may provide boundary cues.

\section{Dachshund}

Dachshund encodes a nuclear protein that appears to affect mainly cells in the posterior margin of the imaginal disc. Without dachshund, these cells (but not those in the anterior aspect of the disc) are fated to become part of the cuticle. In homozygous dachshund-null mutant Drosophila, eyes do not form (Mardon et al., 1994). The expression of dachshund appears to respond to hedgehog stimulation, and it both stimulates and is stimulated by ey expression. Over-expression of dachshund can lead to ectopic compound eye development in a variety of tissues (Shen and Mardon, 1997); it does so in conjunction with eya, with which it physically interacts through conserved molecular domains (Chen et al., 1997). In addition to the eye, dachshund plays an important role during the differentiation of some leg segments, null mutants have characteristic short legs.

\section{Wingless (wg), Wnt}

Wingless is a Drosophila segment polarity gene that is also expressed in the eye and wing discs. In the eye, $w g$ appears to suppress abnormal cellular differentiation in the lateral margins of the eye disc. The inhibition of $W g$ expression by decapentaplegic (dpp), a secreted homologue of mammalian $T G F-\beta$ that is in turn regulated by hedgehog, appears to be required for the initiation and progression of the morphogenetic furrow (Chanut and Heberlein, 1997; Dominguez and Hafen, 1997; Pignoni and Zipursky, 1997). Moreover, the expression of $d p p$ and the suppression of $w g$ both appear to be affected by the expression of eya and eyg (eye gone, a Pax-like gene), two cell-fate specification genes that control the differentiation of cells anterior to the morphogenetic furrow (see Section 2.3.3.2), indicating that there is a close relationship between the molecules regulating the commitment and subsequent differentiation of the cells in the eye imaginal disc (Hazelett et al., 1998).

Multiple mammalian homologues of $w g$ have been identified, of which $W n t 7 b$ appears to be the functional equivalent of wingless during development. Wnt7b is expressed in the dorsal aspect of the developing optic vesicle, the optic stalk and the diencephalon, in a pattern opposite to that of Pax2 (Parr et al., 1993), and is believed to play a role in the establishment of dorsal-ventral axis of the optic vesicle and optic stalk. In view of the function of $w g$ in Drosophila, one may predict that $W n t 7 b$ prevents ectopic or out-of-sequence differentiation of the retinal neurons in the mammalian eye. 


\section{Tailless (tll)}

Tailless is the earliest expressed gene in the regulation of eye development known to date. In Xenopus, the $t l l$ homologue Xtll is expressed in the open neural plate prior to the formation of the optic vesicle, in a pattern that overlaps that of XPax6. Inhibition of Xtll expression disrupts optic vesicle formation and interferes with XPax6 expression (Hollemann et al., 1998), positioning it functionally upstream of XPax6. Like other patterning genes, Xtll continues to be expressed in later stages of the organ development, but within narrowing domains.

\section{$\underline{R x}$}

$R x$ is another family of vertebrate homeobox-containing genes that appear to play a conserved pattern-determining role during early eye development. These genes contain a paired-like domain and other homeodomains that are highly conserved between vertebrate and invertebrate species (Mathers et al., 1997). In Xenopus, The expression pattern of two members of this gene family, XrxI and Xrx2, overlaps those of Xtll and XPax6, but is distinct from the latter. $X r x l$ is expressed in the anterior most part of the neural tube and in tissues that will give rise to the pineal body (Mathers et al., 1997; Casarosa et al., 1997). Unlike XPax6, Xrx is not expressed in the lens, and its expression becomes gradually limited to cells in the ciliary margin as the retina becomes more differentiated. This is consistent with the interpretation that $R x$ is involved in maintaining the survival of retinal progenitor cells, as the ciliary margin is the source of all progenitor cells in the adult Xenopus retina.

A similar pattern of expression has been noted in the mouse, where the mouse $R x$ homologue Mrx is expressed in the anterior neural plate by as early as E7.5, and the expression pattern becomes gradually restricted to the emerging retina and ventral forebrain. The domain of Mrx expression continues to recede as cells in the maturing neural retina exit the proliferative state. During post natal development, Mrx expression is further restricted to photoreceptors and cells in the inner nuclear layer, until it fades completely by P13.5 (Mathers et al., 1997). Artificial over-expression of $R x$ leads to ectopic development of PE, a thickening of the actual PE, and a duplication of the retina. It also appears to cause excessive anterior neural tube development, suggesting that overexpression of Mrx results in more progenitor cells being fated to become anterior neural tube and retinal cells. Conversely, homozygous loss of function Mrx mutant mice do not develop eyes beyond the optic vesicle stage, and have little or no forebrain structures.

The regulation of $R x$ expression, like those of Pax $6, t l l$ and other patterning genes, may be associated with the hedgehog family of signaling molecules. 
2.3.3.2 The cellular specification and differentiation genes.

The genes described in this sub section are involved in the later steps of neuronal differentiation, after the visual progenitor cells are considered biased. It should be readily apparent that there is not a definitive marker which separates the earlier phases of the differentiation process with a later ones, only an abstract distinction based on the extent to which the progenitor cells are believed to be committed to a given fate. The list contains both genes that promote differentiation and those that inhibit the process, because morphogenesis is dependent upon, and modulated by, the combined effect of these regulatory molecules. As with early patterning genes, much has been learned about the differentiation genes through the study of Drosophila eye development; hence, most entries focus mainly on the known functions of such genes in the Drosophila, although examples that suggest these genes are functionally conserved in the vertebrates abound.

\section{Eyes absent (eya, Eya)}

The eya gene appears to act downstream of ey in the inductive sequence of Drosophila eye development (Halder et al., 1998). It encodes a nuclear protein that is expressed in progenitor cells anterior to the morphogenetic furrow and is required for their survival. In vitro experiments have shown that eya interacts with sine oculis and forms a complex with the latter, and likely controls Drosophila eye development through a network of genes involving sine oculis (Pignoni et al., 1997). In the absence of eya, these cells undergo programmed cell death. Bonini and colleagues $(1993,1998)$ have used less severe alleles of eya (where some cells are functionally normal) to determine the time and site of eya action. They found that the eya mutation reduced the number of cells available to be recruited as photoreceptor progenitors. The cells with functional eya went on to form normal-appearing ommatidia, while the rest became apoptotic.

Why cell death? During development, the survival of proliferating cells is dependent upon the successful competition for growth substrates, such as various growth factors. As tissues develop, the requirement for specific types of growth factor changes, thus altering the cellular fate. Differentiation requires the exit from the proliferative state, and may involve the "turning off" of certain responses to growth factors. If cells fail to respond appropriately to differentiation cues within a certain time window, and the growth environment changes, programmed cell death may be the default terminal fate.

An example of the growth factors whose effects the eya gene may have to work against is coded by the argos gene. A secreted protein that contains an EGF motif. In loss-of-function argos mutants, excessive cellular differentiation occurs, resulting in extra 
photoreceptors and cone cells in the adult retina. The over expression of argos through experimental manipulation noticeably inhibited normal photoreceptor and cone cell differentiation (Sawamoto et al., 1994). Like several other genes involved in eye development, such as wingless and Notch, argos is involved in the regulation of wing development as well, adding to the evidence that eye and wing development share numerous early patterning genes and associated factors.

Some parallels of the process above may be seen in the vertebrate retina, where the activation of FGF-tyrosine kinase appears to cause progenitor cells to choose the neuronal fate (instead of becoming PE cells, which seem to be the default fate), while the activation of EGF-tyrosine kinase prevents the multipotent cells from becoming rods (Reh, 1992).

Mammalian homologues of eya have recently been isolated. In the mouse, there appear to be at least three molecules with sequence homology to eya, Eya-1, Eya-2 and Eya-3, which are likely the products of gene duplication. Each of Eya genes has a somewhat different expression domain in the developing embryo (Xu et al., 1997). Eya-1 and Eya-2 appear to be functionally homologous to Drosophila eya, in that they are expressed in the differentiating retina, lens and nasal placode, and are affected by Pax6 expression. Like the Pax genes, all three Eya genes and the Drosophila eya are likely to have regulatory roles outside eye development (Bonini et al., 1998).

\section{$\underline{\operatorname{Ch} x 10}$}

Chx10 is a homeodomain-containing gene of the paired class whose homologues have been identified in the mouse, the chick, the fish (Vsx), and the nematode C. elegans (ceh-10). In the mouse, Chx10 is expressed in the developing and mature eye tissues, first in the neuroepithelial cells of the lateral walls of the optic vesicle, then becoming gradually restricted to the inner nuclear layer of the differentiated retina (Liu et al., 1994). The timing and pattern of Chx 10 expression suggest that it plays several distinct roles during retinal development. It may initially function as a regulator of progenitor cell proliferation in the optic vesicle, then it becomes a specific factor in the bipolar cell differentiation process, and finally it becomes a required molecule for maintain bipolar cell survival in the mature retina (Burmeister et al., 1996).

A defect in Chx10 expression has been found to be the cause of ocular retardation (or), a recessive mutation known to be associated with abnormal cell death and optic fissure defects in the developing eye that lead to retinal and lens atrophy (Truslove, 1962; Robb et al., 1978). As early as 1975, the cause of or developmental defects had been attributed to a lengthening of the G1 period in the cell cycle (Konyukhov and Sazhina), 
this interpretation is supported by evidence in ChxlO null mutants showing that cell proliferation is substantially diminished in the periphery of the mutant retina (Burmeister et al., 1996).

In goldfish, two homologues of Chx10 have been identified thus far, Vsx-1 and $V s x$-2, which have overlapping expression domains. Like their avian and mammalian counterparts, Vsx expression, particularly that of $V s x$-2, is limited to undifferentiated progenitor cells in the developing and mature retina, as well as bipolar cells (Levine et al., 1997; Passini et al., 1997), providing further evidence that the Chxl0 family of genes are involved in maintaining progenitor cells in their proliferative state, perhaps by competing against differentiation promoters; which may also account for their presence in the differentiated bipolar cells.

\section{Notch, Delta, Jagged}

Notch, a Drosophila and vertebrate homologue of C. elegans lin-12 and $g l p-1$, is a highly conserved transmembrane receptor protein believed to play a critical role in mediating cellular differentiation through local cell interactions, both via lateral and inductive signaling pathways (Artavanis-Tsakonas et al., 1995). The fact that Notch appears to be involved in a multitude of developmental pathways suggests that it regulates cellular commitment in anticipation to other differentiation cues, that it may be viewed as a molecular intersection of all such regulatory pathways.

In the developing vertebrate eye, two members of the Notch gene family are expressed, Notch-1 and Notch-2, as well as their membrane receptor ligands Delta and Jagged. Notch-1 and Delta are expressed in undifferentiated progenitor cells in the neural retina, and are thought to influence fate determination and cellular differentiation during multiple inductive events (Ahmad et al., 1997), although there is some debate over whether or not Notch is also involved in the maintenance of differentiated retinal neurons in the mature eye (Ahmad et al., 1995; Bao and Cepko, 1997). Notch-2 expression appears to influence the patterning of non-neural tissues in the eye, such as the PE and the ciliary body. Jagged expression fluctuates widely and appears to be most prominent where tissues are undergoing rapid morphogenesis. For instance, Jagged expression in the ganglion cell axons proportionally decreases as the optic nerve becomes myelinated, leading to the speculation that oligodendrocyte differentiation may be mediated by the Notch-Jagged complex (Wang et al., 1998). 


\section{Atonal (ato), Achaete-Scute, Mash, Math}

Ato belongs to the Achaete-Scute Complex family of proneural genes. In contrast to neurogenic genes whose function is to restrict cell fate, proneural genes make cells competent to respond to inductive cues. Like other members of the gene family, ato encodes a basic helix-loop-helix (bHLH) transcription factor. In loss-of-function ato mutants, R8 cells do not develop, which in turn prevents the differentiation of other receptor cell types (Jarman et al., 1994). It appears that ato biases progenitor cells towards the R8 cell fate along the morphogenetic furrow. The effect of ato on the differentiation of other photoreceptors is indirect, as it plays no role in the inductive pathways of the rest of the progenitors once R8 differentiation has occurred. The inductive effects of ato appears to be modulated by the Notch pathway, but does not depend on it (Dokucu et al., 1996).

Several mammalian homologues of the Achaete-Scute Complex and ato genes have been identified, accordingly named Mash and Math (Guillemot and Joyner, 1993; Akazawa et al.; 1995; Shimizu et al., 1995; Kageyama et al., 1995). These bHLH molecules are expressed in the developing nervous system, including the eye (Mashl), and appear to promote neuronal differentiation. In vitro experiments show that Mashl is involved in the differentiation of late-appearing cell types, such as rod photoreceptors, horizontal cells and bipolar cells, and that in its absence, progenitor cells take on the glial cell fate. However, Mash1 -null mutant mice have no retinal defects, suggesting that there are redundant or alternative regulatory molecules and/or pathways.

\section{$\underline{\text { Rough }}$}

After R8 differentiation, the induction of $\mathrm{R} 2$ and $\mathrm{R} 5$ receptors involves the expression of the rough protein by the progenitors of these two cell types. Rough is a homeodomain-containing transcription factor. Its expression is initially widespread in the morphogenetic furrow, but becomes restricted to R2 and R5 prior to their differentiation. Loss of function rough mutants have disrupted ommatidial organization. Ectopic expression of rough prevents ato expression and inhibits the formation of proneural cluster, suggesting that the two genes have complementary roles in determining the fate of the progenitor cells (Dokucu et al., 1996). The interaction between ato and rough does not appear to involve Notch, as their expression boundary is not disrupted by Notch inactivation. 


\section{Seven-up (svp), bar}

The development of R3, R4, then R1 and R6 require the activities of the svp gene, in the absence of which all four cells would adopt the $\mathrm{R} 7$ fate. The $s v p$ protein sequence shows 77\% overall identity with the human transcription factor COUP/ARP-1 (Fjose et al., 1993). This family of steroid receptor transcription factors is especially conserved within the DNA-binding domain, showing $86 \%-100 \%$ homology between vertebrates and Drosophila. Intriguingly, even though ubiquitous expression of svp causes normal R7 precursors to become R1-R6- like and interferes with R8 differentiation, it also induces the normally non-neuronal cone cell precursors to become R7 photoreceptors (Hiromi et al., 1993). The regulatory pathway in which $s v p$ plays a role clearly performs different functions in different cells. Svp differs from rough in one aspect - it can affect the fate of non-neurons, suggesting that $s v p$ is a more "generic" regulator of cellular differentiation. Prior to R7 differentiation, R1 and R6 cells are further distinguished from $\mathrm{R} 3$ and R4 through the expression of the bar membrane protein.

\section{Sevenless (sev), bride of sevenless (boss), seven in absentia (sina), and associates}

These genes are named according to their similar loss-of-function phenotypes: the absence of R7 receptors. In the ommatidia of each null mutant, R7 progenitors become cone-like. These genes appear to be interconnected components of an inductive pathway that controls R7 differentiation, and are representative of the genes which control the last steps of cellular differentiation.

The sev gene encodes a receptor tyrosine kinase, whose activity alone is sufficient to determine $\mathrm{R} 7$ fate (Basler et al., 1991). It is expressed on the R7 progenitor cell membrane and appears to be activated by boss, a protein containing seven transmembrane loops and which resembles a G-protein-coupled receptor.

Boss is expressed exclusively by R8 cells. Studies have shown that when sev is activated by boss, it initiates a cascade of signal transductions involving a variety of specific and non-specific signal molecules (Hafen et al., 1993). Notably, the Drosophila downstream-of-receptor-kinase (drk) gene encodes an adapter protein containing srchomology-regions 2 and 3 (in SH3-SH2-SH3 configuration common to such molecules), which binds to specific sites on the activated tyrosine receptor kinase sev in order to couple the latter with more downstream effector elements, such as the son of sevenless (sos) protein and a GTPase activating protein Gapl (Olivier et al., 1993; Gaul et al., 1992; ). Sos is a guanine nucleotide-releasing protein (GNRP), which upon activation would catalyze the exchange of GDP for GTP on Ras, thereby activating the latter to trigger the Ras-gated differentiation process. In contrast, GTPase activating protein has 
the exact opposite effect of sos, it hydrolyzes GTP into GDP, thus inhibiting R7 differentiation through the same pathway. Rasl, rather than Ras2, appears to be the effector of R7 differentiation (Fortini et al., 1992).

Sina encodes a zinc finger-containing nuclear protein. A number of molecules have been identified that when combined with sina, seem essential to the induction of R7 differentiation (Carthew et al., 1994). A few of these molecules are also involved in the Rasl activation pathway. For instance rolled (rl), which codes for a MAP kinase, appears to allow sina to overcome the ubiquitous expression of Ras 1, perhaps by acting as a functional link between sina and Rasl.

Evidence suggests that R7 differentiation is not entirely driven by the sev - boss sina pathway. Using an enhancer trap line, Mlodzik and colleagues (1992) have shown that R7-specific markers can still be expressed in mutations where none of the above are functional. However, it is unclear what other genes might be involved.

In vertebrates, much less is known about the specific genetic regulation of the final steps of cell differentiation in the retina. However, a family of murine homologues of the sina gene has been isolated. Not surprisingly, there are several sina homologues (Siah) thanks to gene duplication. Three of the homologues, belonging to two sub-types, appear functional: Siah-1A, Siah-1B and Siah-2 (Della et al., 1993); another two members of the Siah family are pseudogenes with disrupted exons. All three functional Siah genes have highly conserved cDNA sequence, both between themselves (73 to 97.8\%) and with Drosophila sina (66 to 77\%). The expression of Siah-2 is quite high in the retina and forebrain during embryogenesis. It is speculated that the high level of conservation is indicative of conserved function. This has been borne out experimentally in numerous examples given above.

\subsection{Conclusions.}

Evolution offers us a means to explain similarities between seemingly unrelated organisms. It allows us to make falsifiable predictions that we can test in order to understand how these organisms came about. It may not offer the only possible explanation for the origins of the genes and organs we seek to understand, but until a falsifiable alternative mechanism can be shown to better account for the history of the organisms, evolution remains a valuable tool with which we can address the biological questions relating to the function and development of these organisms. 
Given the apparent flexibility with which the eyes have evolved, it is surprising that so much underlying genetic similarities exist between the eyes of even the most unrelated of species. We are left with the impression that beneath the complex façade, the molecular mechanisms controlling the development of widely different organisms are constructed of the same building blocks. More often than one might expect, entire assemblies of such building blocks appear to have been retained en bloc by organisms as they diverged from one another, while tolerating only minor modifications. As investigators who are interested in the regulation of development, we should perhaps feel fortunate that this is the case, since it allows us to pool the knowledge gained from the study of different organisms, and make predictions about novel problems based on the sum of such knowledge. In so doing, the daunting task of deciphering a complex network of regulatory mechanisms guiding development is made somewhat more manageable.

Beneath the diversely colorful surface, life appears to have avoided "re-inventing the wheel" at every turn. 
Chapter 3. Bst, a semi dominant mutation causing retinal defects.

\subsection{Introduction.}

It is evident from the material discussed in the preceding chapter that even in a relatively simple organism such as the Drosophila, numerous genes are involved in the building of a functional eye. Some of the genes (Pax6 for instance), whose expression dramatically affects the entire course of eye development (Halder et al., 1995), are clearly involved in the early steps of the morphogenetic process. In contrast, other genes appear to be more "disposable", whose inactivation often produce no noticeable phenotypic defects, such as in the case of some milder mutant alleles of the mi gene (Steingrímsson et al., 1994) and in Mashl knock-out mice (Tomita et al., 1996). However, these distinctions are not functionally meaningful once we consider that during development, the expression of different genes are interconnected temporally and spatially through various pathways, and that each gene can perform a variety of functions depending on when and where it is expressed. Rather than any inherent differences in functional importance, the effects of genes are a reflection of the positions they occupy within a particular functional cascade, and perhaps the level of redundancy within the cascade. The latter is probably the reason we do not see too many "master genes" in higher order metazoans as we see in more "primitive" organisms. Consequently, the discovery of each new gene offers us a glimpse into the complex relationship between networks of genes, and our understanding of its function only has meaning within the context of such networks.

Given the above, how should we approach the study of development and its genetic regulation? There are currently two options: a forward approach and a reverse approach. In the forward approach, a gene is first identified and characterized by molecular means. If it is suspected to have a role during development, either due to its expression pattern in relevant tissues, or to its molecular similarity with other genes known to play a role during development, the investigator could use a variety of in vitro and in vivo methods to decipher its function. For instance, the gene can be inactivated or expressed ectopically, or one can probe its interaction with other molecules suspected to play a role in the same regulatory pathway. The forward approach provides relatively unambiguous answers. However, due to the limited number of genes currently at our disposal, an investigator is far more likely to encounter a heritable developmental defect which has unknown genetic causes. In such a case, the reverse approach is the only practical course available. The investigator must study the functional outcome of the 
presumed genetic defect in order to formulate a hypothesis with regard to the gene's identity and function. The results from such studies help to identify candidates for the gene and eventually lead to its isolation and full characterization.

The material presented in the succeeding chapters describes a series of experiments designed to define a genetic mutation that produces developmental defects in the mouse retina. While the mutant gene has not yet been isolated, its locus was first identified by Epstein et al. more than a decade ago (1986). It was named Bst for the characteristic white belly spot and kinked tail exhibited by the mutant mouse (Fig. 3.1a). The retinal defects caused by the $B s t$ mutation are believed to lead to varying degrees of optic nerve hypotrophy (Fig. 3.1b). In addition, a subset of Bst mutant mice exhibit exencephaly at birth and many more develop polydactyly (Fig. 3.2). The mutation was intragenic and occurred spontaneously in a colony of C57/BLS mice at the Jackson Laboratory (Bar Harbor, MA). Bst is mapped to Chromosome 16, to a 2-8 cM region that is conserved in human Chromosome 3 hhtp//www informatics jax.org/; Rice et al., 1995; Lunkes et al., 1995). During the course of mapping Bst, it was determined that Bst is inherited semi-dominantly and it is lethal in the homozygous state in the BLKS background. However, its expressivity is more limited in other strains, such as the AKR

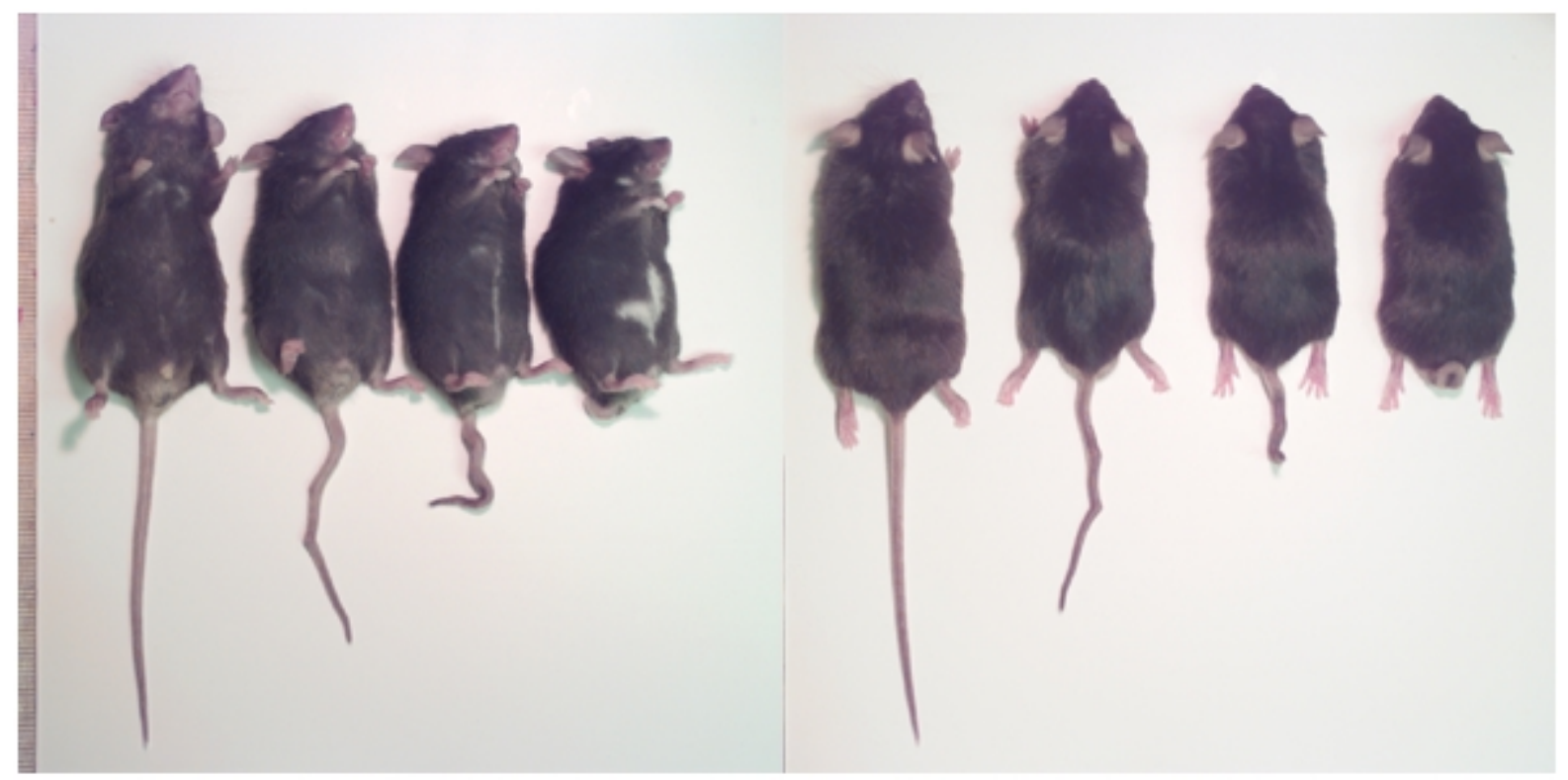

Fig. 3. 1a The range of phenotypes in the $B s t /+$ mice. Four adult male littermates are shown in the supine and prone positions. The extreme left mouse is wildtype, the rest are $B s t /+$. It can be readily appreciated that several characteristics distinguish the Bst/+ mutant from the wildtype. 1) The mutants are slightly smaller than the wildtype. 2) The mutants have varying degrees of kinks in the tail. 3) The mutants have hypopigmented belly spot,although it varies greatly in size. 4) The mutants have hypopigmented feet. 5) The second mouse from the right has 6 toes on the right rear paw. 

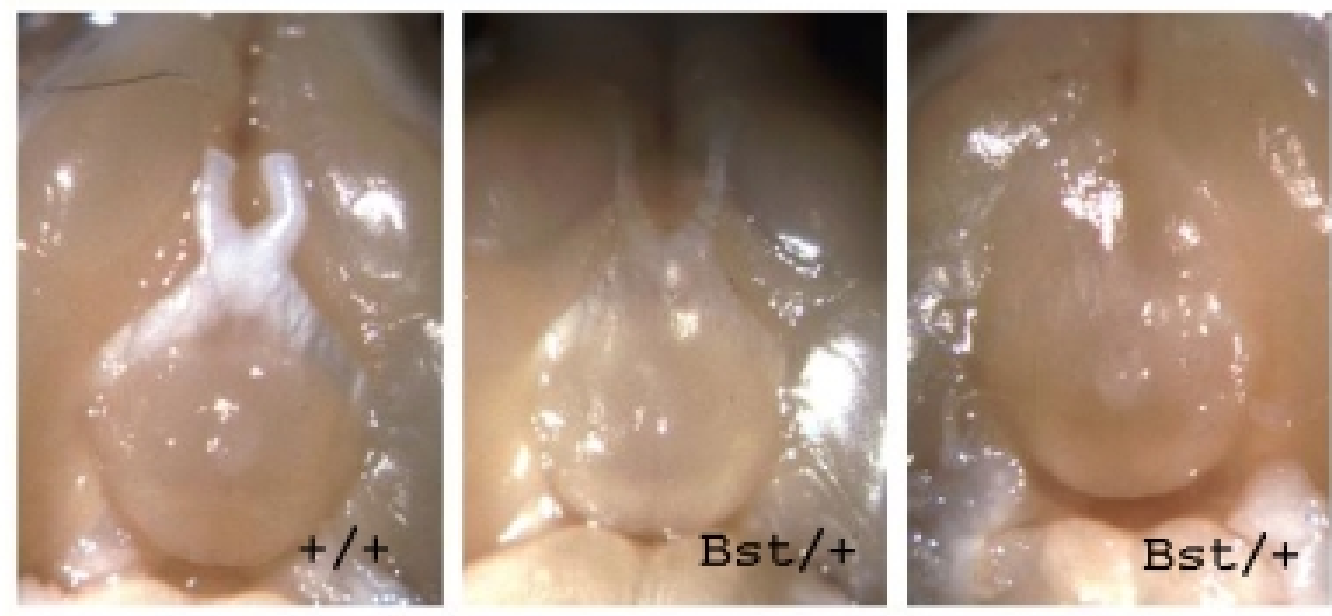

Fig. 3.1b Optic nerve defects in the $B s t /+$ mutant mouse. The mutant mouse in the middle panel has atrophied optic nerves, whereas the mutant mouse in the right panel exhibits bilateral absence of optic nerves
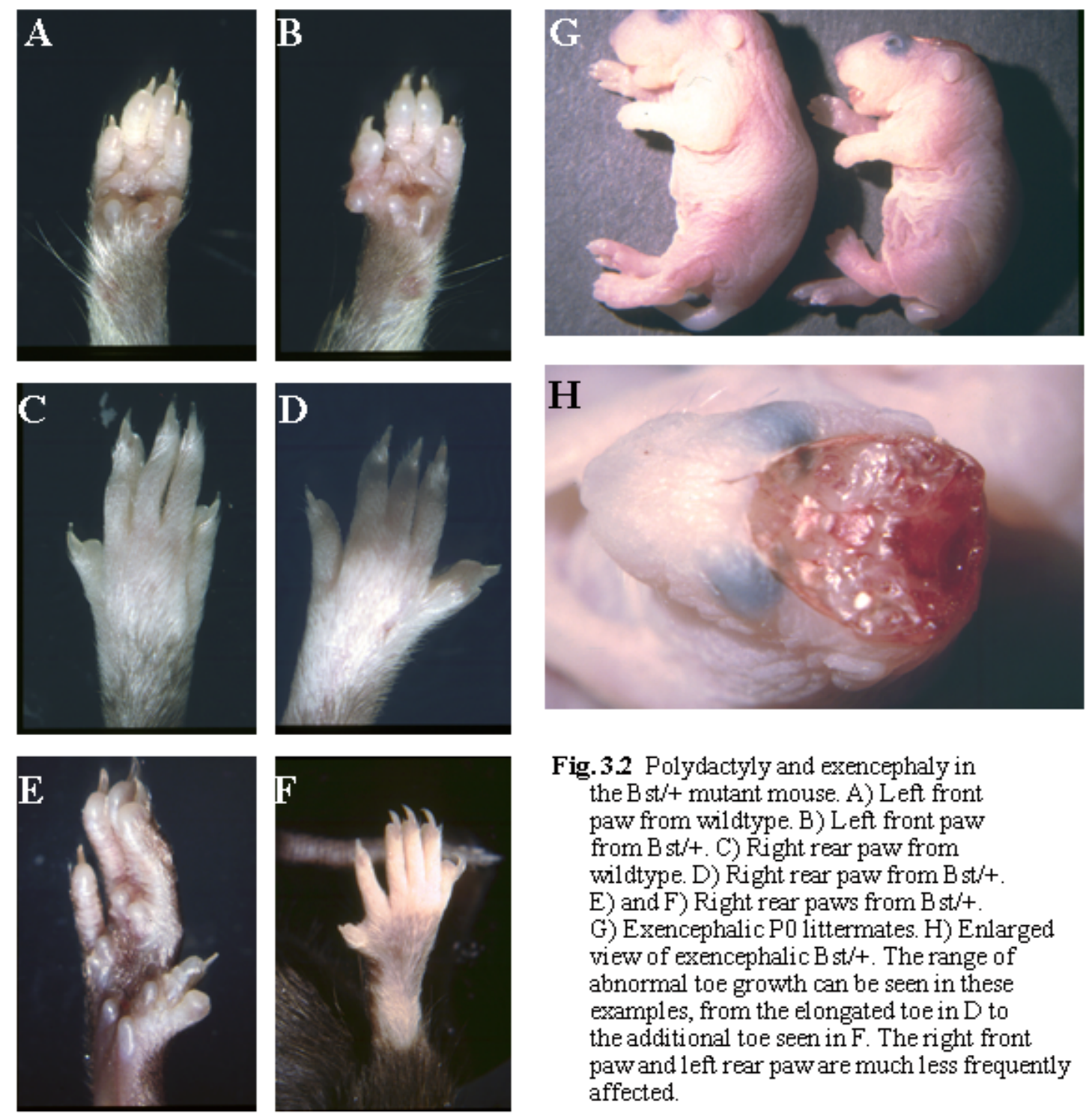

Fig.3.2 Polydactyly and exencephaly in the Bst/ mutant mouse. A) Left front paw from wildtype. B) Left front paw from Bst/ + C) Right rear paw from wildtype. D) Right rear paw from B st/ + . E) and F) Right rear paws from $B$ st/ + . G) Exencephalic P0 littermates. H) Enlarged view of exencephalic Bst/4. The range of abnormal toe growth can be seen in these examples, from the elongated toe in $D$ to the additional toe seen in $F$. The right front paw and left rear paw are much less frequently affected. 
strain used for mapping $B s t$, in which the hemizygous $B s t /+$ mutants are relatively healthy, have less kinks in their tails, and typically exhibit fewer retinal defects and no polydactyly (Rice et al., 1995). Clearly, the Bst gene functions in association with modifier genes in a manner that remains to be determined. Interestingly, the latest examination of the inheritance pattern of the mutant phenotype revealed several chromosomes with "hot spots", where modifier genes could reside, including those containing Pax6 and Wnt7b (Zuo, personal communication). However, even though Bst maps close to several developmentally important genes (Gap43, Hesl), no strong candidate genes have yet to emerge. Nevertheless, the results discussed in later chapters offer some clues with regard to what characteristics a candidate gene for Bst should possess.

Prior to addressing Bst in the context of development, a description of the mutation's manifestation in the adult is necessary to establish a basic understanding of the heritability and expressivity of the mutant gene. The tissues targeted by the Bst mutation, together with the nature of the defects, are central to the formulation of our hypothesis with regard to the activity of the gene, which in turn dictated our experimental design.

\subsection{What is Bst?}

The mutant $B s t$ mouse is visibly smaller than wildtype littermates but not grossly undersized (Fig. 3.1). Aside from the consistent presence of the white belly spot, which varies in size from a few strands of white hair to nearly covering the entire thorax and abdomen, the mutant mouse also has white feet. The tail of the mutant mouse exhibits multiple kinks, which are also variable, from barely perceptible bends in a near normal length tail to a grossly shortened stump that appears to result from numerous severe twists. In addition to the tail anomaly, numerous mutant mice show other skeletal defects as well. For instance, some female mutant mice appear to have a distorted pelvis that prevent them from mating successfully. Many of the mutant mice also have polydactyly. Curiously, there appears to be a limb preference in the susceptibility to polydactyly, in that nearly all mice exhibiting polydactyly have an extra "thumb" on the right rear paw (Fig. 3.2), and somewhat fewer mice have either an enlarged or superfluous "thumb" on the left forepaw. Considerably fewer mutant mice have an extra "thumb" on the left hindpaw, while other digits and the right forepaw appear nearly immune to such defects (Table 3.1). 
Table 3.1 Bst adult phenotype.

\begin{tabular}{lllllllll}
\hline \hline $\begin{array}{l}\text { Number } \\
\text { of } \\
\text { Bst }+\end{array}$ & $\begin{array}{l}\text { Bilateral } \\
\text { normal } \\
\text { pup. reflex }\end{array}$ & $\begin{array}{l}\text { Unilateral } \\
\text { normal pup. } \\
\text { reflex (1) }\end{array}$ & $\begin{array}{l}\text { Bilateral } \\
\text { partial pup. } \\
\text { reflex (2) }\end{array}$ & $\begin{array}{l}\text { Bilateral } \\
\text { absence } \\
\text { pup. reflex }\end{array}$ & $\begin{array}{l}\text { Left } \\
\text { forepaw } \\
\text { anomaly }\end{array}$ & $\begin{array}{l}\text { Right } \\
\text { forepaw } \\
\text { anomaly }\end{array}$ & $\begin{array}{l}\text { Left } \\
\text { rearpaw } \\
\text { anomaly }\end{array}$ & $\begin{array}{l}\text { Right } \\
\text { rearpaw } \\
\text { anomaly }\end{array}$ \\
\hline 94 & $34(36 \%)$ & $30(32 \%)$ & $17(18 \%)$ & $13(14 \%)$ & 4 & 1 & 1 & 12 \\
\end{tabular}

(1) Included in this group are animals that have partial or complete unilateral absence of pupillary reflex.

(2) Included in this group are animals that have partial or complete absence of pupillary reflex in both eyes.

Kinked tail and polydactyly aside, there are other defects that are fatal, such as exencephaly. Exencephalic newborns may survive for hours after birth (Fig. 3.2; Table 3.1). Occasionally, hydrocephalic mutant mice have also been observed, some of which could even survive into adulthood if the symptoms are sufficiently mild. In about one in thirty or more mutants born, there is misalignment of the jaws that makes feeding exceedingly difficult and prevents the normal wear of the teeth. These mice remain undersized and die within the first post natal month if untreated.

In terms of defective functional organs, what is observed in the mutant mice is again variable. A large number of mutant mice are asymptomatic (Table 3.1), while others are afflicted by multiple organ failures. Most noticeable among the organ defects is that of the eye, which Rice et al. (1995) have detected by assessing the pupillary reflex. In this procedure, a penlight is briefly shown into the eye of the test subject after the latter has had time to accommodate to a darkened environment. In the normal individual, this stimulus results in an immediate contraction of the pupil to a minimal aperture, whereas in a symptomatic mutant mouse, the contraction may occur slower, and may stop short of the minimal aperture. In the convention followed by Rice et al., the pupillary reflex is graded according to its final aperture on a scale of 0 to 5 , with "0" denoting normal reflex (i.e., maximum contraction), and " 5 " indicating a complete absence of reflex (i.e., the pupil remains relaxed and open). Hence, a pupillary contraction that nearly reaches minimal aperture would receive a "1" grading, while one which is barely noticeable would receive a "4". In all the animals examined thus far, this grading system has been consistently successful at predicting the extent of retinal defects. Mice that have been graded "5" in a given eye typically have no optic nerve in that eye, while those with more noticeable pupillary contractions also have correspondingly healthier retinas and larger optic nerves. It can be appreciated from Table 3.1 that in a given individual, extreme defects found in one eye does not guarantee that the other eye would be affected at all. However, unlike in the case of polydactyly, there appears to be no side preference in retinal defects. In older mutant mice, the presence of cataract and symptoms of increased 
intra-ocular pressure (appreciably enlarged eye) can often be observed, while others develop circling behaviors suggestive of neural tube and inner ear defects (Cripps and Nash, 1983).

All the defects and symptoms seen in the Bst mutant mouse have been reported in other mutations as well, some of which are listed in Table 3.2. Many of these defects are the result of disrupted development involving the same tissues. For instance, the white belly spot seen in the Bst is typical of mutations which affect neural crest derived melanocytes (Steingrímsson et al., 1994). Cells originating from the neural crest are involved in the development of numerous organs, such as the eyes, the ears, the skin, and the skull (Weston, 1970). One may therefore be tempted to surmise that in a mutant mouse such as the Bst, which exhibits defects in the above organs, the neural crest is the site of the mutant gene activity. However, it is also possible that the neural crest is only one of several tissues affected by the mutation. Since each organ represents the end product of thousands of genes directing the transformation and migration of numerous tissues, and given Nature's proclivity to perform multiple functions with each molecule, it would not be surprising to find numerous genes that affect the development of a common set of organs, but through unrelated pathways. In order to determine the true nature of $B s t$ 's activity during development, we have to not only consider which organs and tissues are affected, but also define the specific events that are the source of the defects. For instance, if we find that there is abnormal growth in the Bst eye shortly after the

Table 3.2 Retinal defects and associated mutations

\begin{tabular}{|c|c|c|c|c|}
\hline Mutation & Eye defects & Other defects & Gene function & gene \\
\hline Bst & $\begin{array}{l}\text { Delayed retinal development, } \\
\text { optic fissure fusion anomaly. }\end{array}$ & $\begin{array}{l}\text { Pigmentation, neuropore } \\
\text { closure related defects, } \\
\text { polydactyly. }\end{array}$ & unknown & unknown \\
\hline Sey & $\begin{array}{l}\text { Retinal atrophy, no lens, fusion } \\
\text { anomaly, small eyes. }\end{array}$ & $\begin{array}{l}\text { None obvious in } \\
\text { vertebrates. }\end{array}$ & $\begin{array}{l}\text { Cell fate specification } \\
\text { and maintenance. }\end{array}$ & Pax6 \\
\hline Krd & $\begin{array}{l}\text { Optic stak fissure fusion } \\
\text { anomaly, retinal and optic neve } \\
\text { atrophy. }\end{array}$ & $\begin{array}{l}\text { Brain, cerebellum, ear, } \\
\text { kidney defects. }\end{array}$ & $\begin{array}{l}\text { Ventral retina, optic stalk } \\
\text { specification. }\end{array}$ & $\operatorname{Pax} 2$ \\
\hline Hesl & $\begin{array}{l}\text { Delayed optic fissure fusion, } \\
\text { premature RGC differentiation, } \\
\text { retinal, lens and corneal defects. }\end{array}$ & Neural tube defects. & $\begin{array}{l}\text { Suppress premature } \\
\text { retinal ganglion cell } \\
\text { differentiation }\end{array}$ & Hes-1 \\
\hline wingless & Microphthalmia in Drosophila. & $\begin{array}{l}\text { CNS, metameric body } \\
\text { pattern defects in } \\
\text { Drosophila. }\end{array}$ & $\begin{array}{l}\text { Axial specification, } \\
\text { prevention of out-of- } \\
\text { sequence differentiation. }\end{array}$ & Wnt7b \\
\hline$X t$ & $\begin{array}{l}\text { Variable phenotype, small to no } \\
\text { eyes, lens induction anomaly. }\end{array}$ & Kinked tail, polydactyly. & $\begin{array}{l}\text { Transcription factor } \\
\text { regulated by } S H H\end{array}$ & Gli3 \\
\hline
\end{tabular}


formation of the optic vesicle, prior to any substantial involvement of neural crest-derived cells in the formation of ocular structures, then we cannot consider defects involving the neural crest as the primary cause of the Bst mutant phenotype, even if such defects do in fact occur.

\subsection{The Bst retina.}

The eyes of the Bst mutant mouse are somewhat smaller than those of the wildtype, but not disproportionately so considering the overall smaller stature of their hosts. The retinas of the mutant mice are on average $10 \%$ smaller in surface area than those of the wildtypes (Rice et al. 1997) and often exhibit surface irregularities (Fig. 3.3). In cross sections, a range of mutant retinal phenotypes can be seen, from near normal to

severe hypotrophy and dysmorphism, to include such anomalous features as coloboma, folding, rosettes, and the occasional presence of ectopic ganglion cell axons (Rice et al., 1997). In animals which have undersized or absent optic nerves, the retinal ganglion cell layer is correspondingly thinner and have fewer cells. This hypotrophy is typically seen in the inner plexiform layer and inner nuclear layer as well; whereas the outer nuclear layer appears far less vulnerable to the insult.
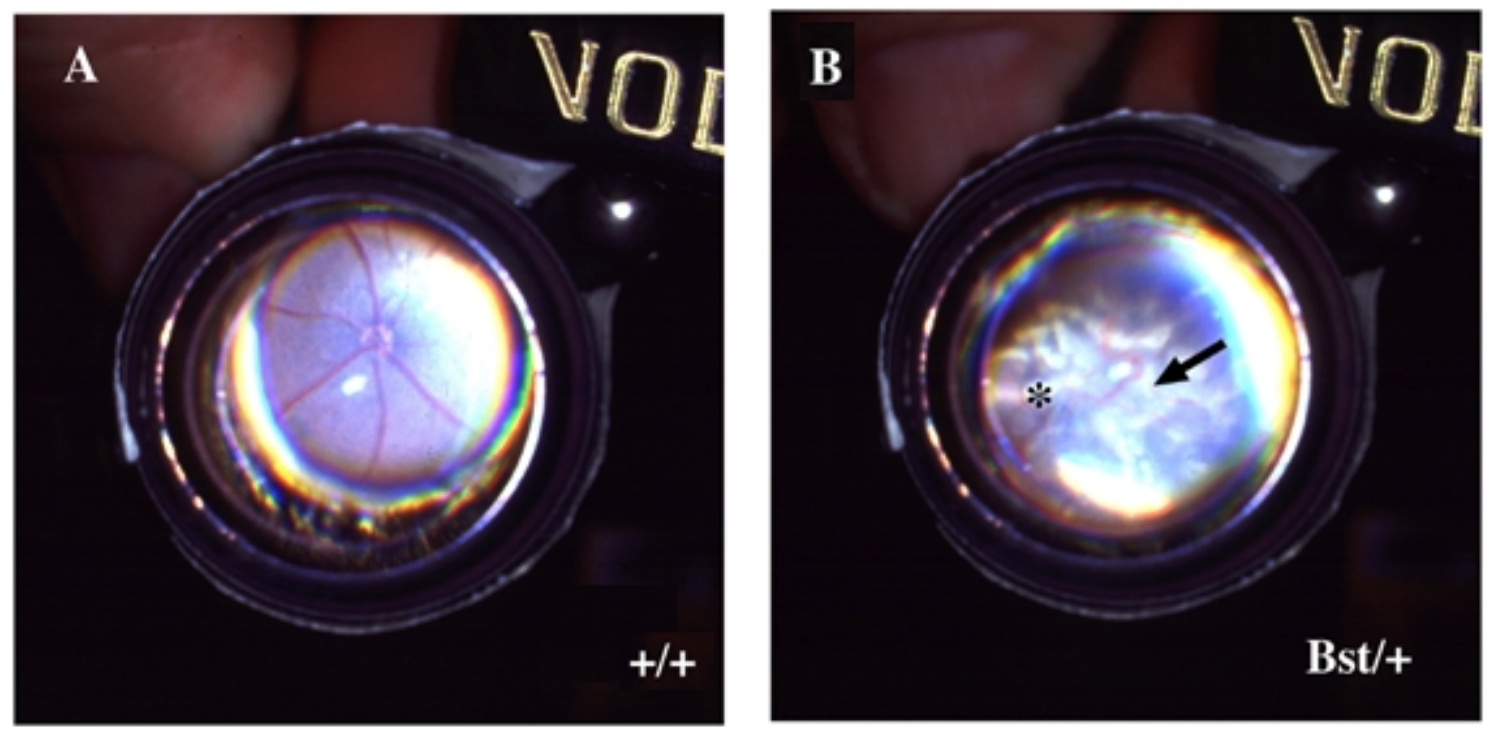

Fig. 3.3 Fundus camera view of the $B s t /+$ retina. A) Wildype. B) $B s t /+$. Arrow points to irregularities on the retinal surface, indicative of retinal detachment. Asterisk marks irregular arterioles in the $B s t /+$ retina. 
A particular feature of the mutant retina that stands out among other anomalies is the abundance of structural defects near the optic fissure (Fig. 3.4). As the optic cup is formed from the collapse of the optic vesicle, it folds ventrally and forms the optic fissure near the mid-line. Fusion of the fissure begins shortly after the margins make contact. Fissure fusion is thought to play a role in guiding the exit of ganglion cell axons into the optic stalk (Silver and Sidman, 1980, Otteson et al., 1998). In the mutant eye, there are

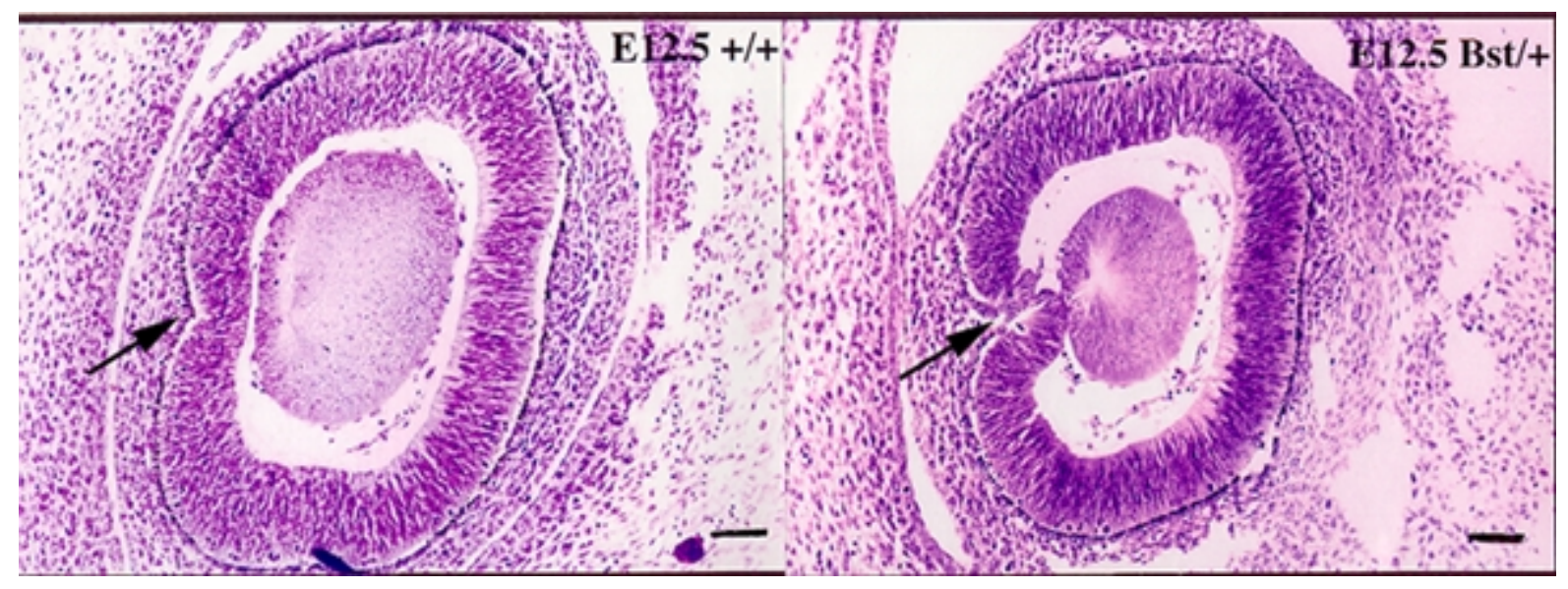

Fig. 3.4 Optic fissure fusion anomaly in the Bst/+ retina. Sagittal sections of E12.5 retinas stained with cresyl violet are shown. Arrows mark the positions of the optic fissure. The optic fissure of the Bst/+ mutant is clearly not fused and appears to have buckled due to excess growth. Scale bars represent $100 \mu \mathrm{m}$.

often folds of the neural retina adjacent to the optic fissure, which typically result in retinal detachment from the underlying pigmented epithelium (upon dissection, such retinas separate readily from the PE without mechanical input). These characteristic "ridges" are commonly seen in mutations that impede optic fissure fusion, for instance in microphthalmia (mi) mutants (Jackson, 1981; Hero, 1989) and Hes 1 knockouts (Tomita et al., 1996). For this reason, Rice and colleagues suspected that optic fissure fusion is abnormal in the Bst mutant mouse, and that the fissure fusion process may be the site of the mutant gene action (1997).

\subsection{The study of Bst's role during eye development.}

What can we do to characterize the role of Bst without knowing what the gene is? The first step we chose to take was to determine the time frame within which $B s t$ produces its major effects. Rice and colleagues determined that much of the retinal degeneration in the Bst/+ mutant mice occurred prenatally (1997); therefore the question 
became: How early can we detect a defect in the developing eye of the mutant embryo that can account for the anomalies seen in the adult?

What specific defects should we look for? In planning our approach, we formulated our hypotheses based on what is known about other mutations where similar or identical features are observed in the retina. For instance, abnormal cell proliferation and cell death at the optic fissure has been linked to the delay or absence of fissure fusion in the mi mutant mouse (Hero, 1989; Hero et al., 1991; see also Silver and Hughes, 1973, 1974). Delayed cell proliferation has been linked to the retinal defects seen in the or mutant mouse (Konyukhov and Sazhina, 1975; Burmeister et al., 1996). Therefore, cell proliferation and cell death were considered to be potential targets of Bst gene action. In addition, we believed that once we had learned the time frame within which Bst is likely to regulate a particular aspect of eye development, it was important to examine the effects of $B s t$ in the context of other genes known to control the same aspect of eye development during the same period. In so doing, we would have taken the first step towards establishing the position of Bst within the genetic regulatory cascade controlling eye formation.

The specific questions we posed were: 1) When does Bst play a role during eye development? 2) Which tissues are affected by the mutation? 3) What are the defects in these tissues? 4) What are the effects of Bst on other genes expressed within the same spatial temporal coordinates and are there any links between them? 5) How does what we learn about $B s t$ fit with what we know about eye development? These questions were addressed in the experiments discussed in Chapters 4 and 5. 
Chapter 4. Disrupted retinal development in the embryonic Bst mutant mouse.

\subsection{Introduction.}

The retina provides an opportunity to study how a complex neuronal structure arises from simple embryonic Anlage, both in terms of the anatomical transformations which define the morphogenetic process and the genetic determinants which trigger and regulate these events. As more genes are becoming known to play critical roles during eye development, it is increasingly evident that regulation of ocular morphogenesis is a function of the interactions between a network of evolutionarily conserved genes, such as Pax6, Pax2, Chx10, Mitf, Hes1, etc. (see Chapter 2). However, there are numerous gaps in our understanding of how these genes ultimately translate into morphologic entities. For instance, Pax2 inactivation has been shown in both human and mouse to correlate with severe retinal defects, possibly through interference with proper optic stalk fusion (Keller et al., 1994; Favor et al., 1996; Torres et al., 1996; Otteson et al., 1998), yet we do not know the Pax2 target molecule(s) in the retina nor the mechanism by which Pax2 may affect optic stalk fusion. Only through the identification of additional genes that affect eye development, and by examining their roles in relation to genes with known functions, will we be able to piece together the network of inductive and regulatory gene expression cascades through which ocular development is initiated and guided.

The murine autosomal semi-dominant mutation, Belly spot and tail (Bst), is likely to involve a gene that plays a role within such a regulatory cascade. The Bst mutant phenotype is characterized by abnormal development of multiple organs, including the neural retina, skeleton, and coat pigmentation (see Chapter 3). Bst has been mapped to Chromosome 16, within a region that is conserved on human Chromosome 3 (Epstein et al., 1986; Rice et al., 1995).

Given the structural anomalies observed at the optic fissure in $B s t /+$ mice, we have proposed a link between abnormal optic fissure closure and the consequent disruption of retinal development in the Bst/+ retina. Past studies have shown that timely formation and fusion of the optic fissure are essential components of normal development of the retina and optic nerve; significant disruption of these events often results in severe structural defects (Mann, 1964; Theiler et al., 1976; Silver and Robb, 1979; Jackson, 1981; Hero, 1989; Hero, 1990; Hero et al., 1991; Otteson et al., 1998). However, the cellular and molecular mechanisms controlling optic fissure closure are not yet understood. The characterization of the Bst mutation may provide important insight into the inductive and regulatory processes that are essential to the formation and fusion of the optic fissure. 
Moreover, the diversity of organs consistently affected by the $B s t$ mutation suggests that the normal Bst gene product is a likely component of many molecular interactions underlying divergent developmental pathways.

In this study, we have examined embryos from post-coïtal day 9.5 (E9.5) through E13.5. We describe the earliest observable differences between hemizygous mutant $(B s t /+)$ and wildtype $(+/+)$ littermates in terms of retinal size and morphology. In order to test whether these differences are due to abnormal cellular proliferation or cell death, we have quantified and compared mitotic indices and the level of pyknosis between mutant and wildtype littermates in the neural retina. Our results show that retinal growth is delayed in $B s t /+$ embryos by as early as E10.5, while cellular proliferation and cell death occur at essentially normal rates. Also, optic fissure fusion does not occur in the Bst/+ retina until well after the fissure margins have made contact. Importantly, we have examined the time frame within which retinal cells are born, and documented a marked delay in the time $B s t /+$ retinal neuroblasts leave the mitotic cycle in comparison to that witnessed in the $+/+$ retina. Lastly, the severity of retinal defects is variable among $B s t /+$ embryos; the variability is consistent with the range of defects previously observed in older embryos and postnatal mice (Rice et al., 1997). These results indicate that the Bst mutation affects retinal morphogenesis during early development, perhaps through delayed neuronal differentiation, which leads to other anomalies in the retina such as impeded fissure fusion.

\subsection{Materials and methods.}

\subsubsection{Animals.}

Hemizygous Belly spot and tail (Bst/+) mice on the C57BLKS/J background were obtained from the Jackson Laboratory (Bar Harbor, ME) and maintained in our colony. Animals were fed with a standard diet and maintained on a $14 \mathrm{hrs}: 10 \mathrm{hrs}$ light and dark cycle. The animals were screened for pupillary reflex anomaly by shining a penlight into their dark-adapted eyes (Rice et al., 1997). The extent of pupillary contraction was graded on a scale of 0 to 5 , with " 0 " denoting normal contraction and " 5 " indicating no contraction. This screening method provided a simple means to identify $B s t /+$ mice with defective retinal phenotypes. The affected individuals were mated with wild type $(+/+)$ mice to produce timed-pregnant embryos for our study. In general, male $B s t /+$ mice 
paired with $+/+$ partners proved to be most productive. Females were checked daily for vaginal plugs. Midnight of the day on which a plug was detected was considered embryonic day zero (E0) for the resulting litter.

\subsubsection{Embryo collection.}

At E9.5, E10.5, E11.5, E12.5, E13.0 and E13.5, pregnant dams were sacrificed by cervical dislocation. Embryos were dissected and immersion fixed in either acetic acid/ ethanol (1:3) or $4 \%$ paraformaldehyde in $0.1 \mathrm{M}$ phosphate buffered saline (PBS, $\mathrm{pH}=7.3$ ) depending on the requirement of the experimental regimes to follow. The age of the embryos was visually verified by comparing their size and anatomical features to standard references (Rugh, 1968; Theiler, 1972; Kaufman, 1992). In E11.5 and older litters, genetically $B s t /+$ embryos could be identified based on their noticeably shorter tails. In younger litters, the genotype of embryos was inferred from measurement of their crown to rump length and their morphologic features. Given that $B s t /+$ embryos were always smaller than their $+/+$ littermates in older litters, those who were visibly undersized in the younger litters were identified as $B s t /+$. This presumption was borne out by the consistency of the numeric proportion of $B s t /+$ among the litters examined. Control litters from C57BLKS $/ \mathrm{J}+/+\times+/+$ matings were used to provide references for the $+/+$ phenotype at all ages. We noted the number of embryos and resorptions in each litter in order to keep track of changes in litter size and composition between groups during the gestation. The age and number of subjects collected were as follows: E9.5 (5 experimental litters with 41 embryos; 2 control litters with 18 embryos); E10.5 (7 experimental litters with 60 embryos; 4 control litters with 35 embryos); E11.5 (4 experimental litters with 34 embryos; 1 control litter with 7 embryos); E12.5 (3 experimental litters with 26 embryos; 1 control litter with 7 embryos); E13.5 (2 experimental litters with 18 embryos; 2 control litters with 16 embryos).

\subsubsection{Histological methods.}

Embryos intended for histological examination were embedded in paraffin, sectioned at $6 \mu \mathrm{m}$ in the sagittal phase and mounted on slides (Superfrost + , Fisher). This plane of section allowed sequential analysis of the optic fissure over its entire length. Typically, a +/+ embryo was embedded next to a $B s t /+$ littermate to allow parallel processing and facilitate comparison. A preliminary scan of the embryos was performed by examining cresyl-violet (CV)-stained sections spaced between $90 \mu \mathrm{m}$ and $120 \mu \mathrm{m}$ apart. This allowed the determination of the range of sections containing the eyes and the 
orientation of the latter. In order to compare positionally equivalent retinal sections between groups, each eye was conceptually divided into three regions in the axis perpendicular to the plane of section: 1) The lateral third of the eye, extending from the iris through the center of the lens, contained the distal retina. 2) The middle retina, defined within the equatorial third of the eye and distinguished by having large vitreous space and cells oriented parallel to the plane of section. 3) The medial third of the eye, representing the floor of the optic cup, contained the proximal retina, where cellular orientation became increasingly perpendicular to the plane of section as sections were taken closer to the optic disc. Once the position of these retinal regions had been established, closer examinations were performed using sections spaced no more than 30 $\mu \mathrm{m}$ apart within the regions. Comparative analyses were principally carried out on the middle retina for the following reasons: 1) The middle retina is substantially perpendicular to the plane of section, and is thus relatively insensitive to variations introduced by subtle anglular differences between the plane of section and retinal curvature. 2) Given the above, the differences in the cross-sectional area of the middle retinas from two individuals can be used as a means to estimate the overall dimensional differences between their respective retinas.

\subsubsection{BrdU-labeling of mitotic cells.}

The thymidine analog bromodeoxyuridine (BrdU; Sigma, St. Louis, MO) was used to both measure the mitotic index (short survival protocol) and identify cells that had exited the cell cycle at specific time points during development (cell birthdating; long survival protocol).

In the short survival protocol, timed pregnant dams were intraperitoneally injected with BrdU at $50 \mu \mathrm{g} \mathrm{BrdU/gm} \mathrm{body} \mathrm{weight} \mathrm{one} \mathrm{hour} \mathrm{prior} \mathrm{to} \mathrm{sacrifice.} \mathrm{The} \mathrm{embryos} \mathrm{were}$ dissected in cold $0.9 \% \mathrm{NaCl}$, phenotypically assessed and measured, then immersion fixed in 3:1 acetic acid/ ethanol, embedded and sectioned as described above. Subsequently, a subset of the sections containing the retina were stained for the detection of BrdU incorporation using the method developed by Hamre and Goldowitz (1995) in the following manner:

The slide mounted paraffin sections were gently flattened using downward pressure, then placed on slide warmer to melt the paraffin. Each slide was then deparaffinized through 3 solutions of xylene at 3 minutes each, followed by 3 solutions of $100 \%$ ethanol at 2 minutes each, followed by 3 minutes in a 90\% ethanol solution, and 3 minutes in a $70 \%$ solution, and lastly 3 minutes in a $50 \%$ ethanol solution. After 
deparaffinization, the tissues were treated with pepsin and $\mathrm{HCl}$ in preparation for antiBrdU immuno-reaction. The slides were placed in a coplin jars containing $0.04 \%$ pepsin solution in $1 \mathrm{~N} \mathrm{HCl}$ and gently rotated for 10 minutes. The slides were then transferred to separate coplin jars containing a $2 \mathrm{X} \mathrm{SSC(standard} \mathrm{sodium} \mathrm{citrate)} \mathrm{solution} \mathrm{that} \mathrm{was} \mathrm{pre-}$ heated to $80^{\circ} \mathrm{C}$. The jars containing the slides were incubated in an $80^{\circ} \mathrm{C}$ water bath for 5 minutes, after which the slides were transferred to a $70 \%$ ethanol solution preheated to $60{ }^{\circ} \mathrm{C}$ in which they were allowed to stand for 2 minutes. The immersion solution was then replaced with PBS and slides were allowed to stand for 3 minutes at room temperature, followed by a 3 minute treatment of $10 \% \mathrm{H}_{2} \mathrm{O}_{2}$ in PBS, after which the slides were transferred to PBS-T ( $3 \mathrm{ml} 100 \%$ Triton per $1 \mathrm{~L} \mathrm{PBS}$ ). Primary antibody (antiBrdU, Becton Dickinson, San Jose, CA) was applied overnight in a solution consisting of 4\% anti-BrdU stock and 5\% NGS (new goat serum) in PBS-T. Sufficient solution was applied to cover all tissues (typically $500 \mu \mathrm{l} / \mathrm{slide}$ ), and a rubber cement "ring" was occasionally used to help maintain the solution over the tissue. The slides were incubated within a sealed plexiglass chamber liberally lined with wetted paper towels. After the overnight incubation, the slides were rinsed through 3 changes of PBS-T at 10 minutes each. The secondary antibodies are then applied, which consisted of a PBS-T solution containing 4\% mouse IgG (Vectastain ABC kit, Vector Laboratories, Burlingame, CA) and 5\% NGS. The secondary labeling reaction was allowed to occur for 30 minutes, after which the slides were rinsed in PBS-T for 10 minutes. The signal detection procedure was performed according to the manufacturer's instructions. Following the process, the slides were rinsed 3 times in PBS at 10 minutes per rinse, then transferred to APB (alkaline phosphate buffer) for 10 minutes, and finally reacted with $\mathrm{DAB}(30 \mathrm{mg}$ diaminobenzidine in $60 \mathrm{ml} \mathrm{APB}$, catalyzed by $800 \mu \mathrm{l}$ of $0.3 \% \mathrm{H}_{2} \mathrm{O}_{2}$ just prior to use) for 10 to 20 minutes. As soon as the tissues show the tell-tale brown taint of the positive reaction, while the negative control tissues (subjected to identical treatment minus the primary antibody) remained clear, the slides were transferred through 6 PBS rinses at 5 minutes each. Once the immunoreaction was positively verified under microscopic examination, the slides were optionally counter-stained with $\mathrm{CV}$, dehydrated through ethanol and xylene solutions (in reverse order from that of deparaffinization), and coverslipped using Permount.

The long survival protocol was identical to the short protocol in its preparations, with the exception that litters were allowed to be carried full term after BrdU injection. At birth (P0), newborns were collected, genotyped according to tail morphology, anesthetized on ice, and transcardially perfused with 3:1 acetic acid/ethanol. The eyes were dissected and hemisectioned in the medial-lateral axis (from the optic nerve head to 
the iris). The hemiretinas were embedded in paraffin on the cut surface and sectioned at 6 $\mu \mathrm{m}$. Each of these sections contained a continuous center-to-periphery representation of the retina. Sections were mounted on slides and immuno-reacted for BrdU using the same protocol described above. The number of animals examined at each time point is as follows: E9.5 (2 +/+, $4 \mathrm{Bst} /+)$; E10.5 (4 +/+, $5 \mathrm{Bst} /+)$; E11.5 (4 +/+, $3 \mathrm{Bst} /+)$; E12.5 (2 +/+, 2 Bst/+).

\subsubsection{TUNEL analysis of dying cells.}

Timed-pregnant females from $B s t /+\times+/+$ mating were sacrificed at E11.5, E12.5, and E13.5 for the analysis of cell death in mutant and normal retinas. The embryos were immersion-fixed in $4 \%$ paraformaldehyde, embedded in paraffin, and sagittally sectioned at $6 \mu \mathrm{m}$. The position and size of the eyes were determined using sample sections taken at $180 \mu \mathrm{m}$ intervals and stained with CV. Subsequently, sections containing distal, middle and proximal portions of the retina were mounted on slides and underwent TUNEL reaction following manufacturer's recommendations (Oncor, Gaithersburg, MD).

Briefly: The slides containing paraffin-embedded tissues were flattened and deparaffinized and rinsed in PBS. The slides were treated with proteinase-K $(100 \mu \mathrm{g} / \mu \mathrm{l})$ for 15 minutes at room temperature, followed by 2 washes in $\mathrm{dH}_{2} \mathrm{O}$ and 1 rinse in PBS. On positive control slides, DNase was added as per manufacturer instructions. Subsequently, all slides were treated with $2 \% \mathrm{H}_{2} \mathrm{O}_{2}$ in PBS for 5 minutes, followed by 2 rinses in PBS for 5 minutes each, and covered with equilibration buffer $(75 \mu 1 /$ slide $)$ and coverslipped for 10 minutes at room temperature. After removing the coverslips and wicking off excess buffer, TdT enzyme reaction buffer was quickly added to the slides (75 $\mu 1 /$ slide) and the coverslips reapplied. The incubation was carried out in a humid chamber for 1 hour at $37^{\circ} \mathrm{C}$, during which the pre-wash buffer was also pre-warmed to $37^{\circ} \mathrm{C}$ in a screw-capped coplin jar. After incubation, the slides were transferred to the prewarm buffer, and the capped jar was agitated for 15 seconds, and allowed to stand for 10 minutes at room temperature. The slides were then rinsed in 3 volumes of PBS for 5 minutes each, after which they were placed in a pre-chilled coplin jar containing $70 \%$ ethanol and allowed to stand overnight at $-20^{\circ} \mathrm{C}$. Next, the slides were rinsed 3 times in PBS, and $75 \mu \mathrm{l}$ of anti-digoxigenin-peroxidase solution was applied to each slide. The slides were coverslipped and incubated in a humid chamber for 30 minutes at room temperature. After the incubation, the slides were again rinsed in PBS, and reacted with DAB (see BrdU reaction). After rinsing and visual assessment of the reaction results, the 
slides were counter-stained with methyl green, dehydrated, and coverslipped with Permount.

The following embryos were examined: E11.5 (5 +/+, 2 Bst/+); E12.5 (1 +/+, 1 Bst/+); E13 (2 +/+, 2 Bst/+); E13.5 (2 +/+, 2 Bst/+).

4.2.6 Analysis of retinal morphology, cell density, and proliferative index.

Detailed retinal examination was performed only on those embryos for which genotype was not in doubt. This was straightforward for E11.5 litters and beyond, as older $B s t /+$ embryos exhibited characteristically shorter tails. In all cases, these embryos were also smaller than all $+/+$ littermates. Thus, in younger embryos we based our genotypic determination on the size of the embryo. At E9.5 and E10.5, we limited our analysis to embryos that clearly belonged to either the larger, presumptive +/+ group, or the obviously smaller, presumptive $B s t /+$ group (see Results).

Each eye was analyzed using representative sections from the three retinal regions as defined above. Retinal sections were traced using a camera lucida attached to a Zeiss microscope. The following analyses were performed on the camera lucida drawings: 1) Retinal morphology: The extent of optic fissure formation and fusion, the presence of retinal folds or other anomalies in the eye were noted. 2) Retinal size: A digital tablet was used to measure the retinal area. 3) Ocular size: The number of sections between the iris and optic stalk was used to estimate the length of the eye. When combined with the retinal area data, these results provided a means to gauge the three-dimensional differences between Bst/+ and +/+ retinas. 4) Retinal cell density: Cell counts were taken in $900 \mu \mathrm{m}^{2}$ sampling bins evenly spaced between $60 \mu \mathrm{m}$ to $100 \mu \mathrm{m}$ apart within a retinal section, at positions corresponding to ventral, dorsal, nasal and/or temporal aspects of the retina. The sampled cell densities were compared between groups. 5) Cell proliferation index: The percentage of retinoblasts labeled one hour following the BrdU pulse constituted the proliferation index (PI). The same sampling procedure described for cell counting was used to tabulate the number of BrdU-labeled cells. PI was obtained by dividing the average density of BrdU-labeled cells by the average cell density of adjacent CV-stained sections. Regional PI were also compared between groups (e.g., proximal and distal, nasal and temporal). 6) Cell birth index: The percentage of retinoblasts exiting the mitotic cycle at a given embryonic age constitutes the cell birth index (BI) at that age. The number of cells exiting the mitotic cycle was estimated by counting strongly labeled nuclei in serial sections through the retina of $\mathrm{P} 0$ animals BrdU-dosed at the given embryonic age. $\mathrm{BI}$ was obtained by dividing the estimated number of cells exiting the mitotic cycle by the total 
number of cells (retinoblasts) in the retina at the corresponding embryonic age. The latter was derived from the cell density and retinal size measurements described above. The calculation of the total number of strongly labeled cells at $\mathrm{P} 0$ and the total number of cells in the embryonic retina were carried out by the method of Abercrombie, using a correction for counting split nuclei (Davies, 1978). The counting particle was determined to be 5-6 $\mu \mathrm{m}$ in these calculations. 7) Asymmetry: Based on retinal size and the state of fissure fusion, we recorded the number of embryos that had measurable bilateral asymmetries. In E10.5 embryos, asymmetry was noted when one eye exceeded the other by $25 \%$ or more in ocular length. In E11.5 through E13.5 embryos, retinal asymmetry was characterized by differences in the extent of optic fissure fusion (partial vs. complete).

\subsubsection{Image capture and processing.}

Digital photomicropraphs were obtained using a Nikon Microphot-X microscope coupled to a Kodak DCS 460 digital camera. A Power Macintosh with Photoshop 3.0.5 (Adobe) was used for image capture and figure composition. Additional figures were obtained using a SPOT digital camera (Diagnostic Instruments Inc.) attached to a Zeiss microscope. The images were captured on a Pentium PC running Photoshop 4.0.

\subsection{Results.}

4.3.1 Gross observation of embryonic development.

\subsubsection{E9.5.}

At this stage during development, the diminutive tail of the $B s t /+$ is not yet observable. However, about a third of the embryos in each $B s t /+\times+/+$ litter are visibly smaller than the rest (14 of 41 embryos from 5 litters, ranging from 1/2 to $4 / 5$ in size compared to littermates), while only 1 embryo from the controls (18 embryos from 2 litters) is comparably undersized. Since $B s t /+$ embryos are noticeably smaller than $+/+$ in older litters and make up about a third of all embryos (Table 4.1), the majority of the smaller embryos seen at E9.5 are presumably $B s t /+$. We have observed a single resorption in the $B s t /+\times+/+$ litters and none in the control litters. 


\subsubsection{E10.5.}

At this age, embryos from $B s t /+\times+/+$ litters can be visually segregated into three groups: 1) normal ( $\geq 4 \mathrm{~mm}$ crown to rump length), 2) small ( $<4 \mathrm{~mm}$ ), and 3) diminutive $(\leq 3.5 \mathrm{~mm}$; Table 4.1). Groups 2 and 3 make up about $40 \%$ of the total number of embryos and have little overlap between them. This proportion approximates that of the $B s t /+$ embryos observed in older litters. In contrast, only 1 in 16 embryos has been found to be noticeably smaller than normal in the $+/+x+/+$ control litters. Save for the small size, the

Table 4.1 Embryonic Bst litter composition

\begin{tabular}{|c|c|c|c|c|c|c|c|}
\hline Age & Mating scheme & Total litters & $\begin{array}{l}\text { Average litter } \\
\text { size }\end{array}$ & Total $n^{a}$ & $\mathrm{Small} / B s t^{b}$ & $\begin{array}{l}\text { Exencephalic/ } \\
\text { diminutive }^{c}\end{array}$ & Asymmetry \\
\hline E10.5 & $\mathrm{Bst} /+x+/+$ & 4 & 9.00 & 36 & $37.14 \%(13)$ & $14.29 \%(5)$ & $23.08 \%$ \\
\hline E10.5 & $+1+x+1+$ & 3 & 9.67 & 16 & $6.25 \%(1)$ & 0 & \\
\hline E11.5 & Bst $/+\times+1+$ & 4 & 8.50 & 26 & $30.77 \%(8)$ & $7.69 \%(2)$ & \\
\hline E11.5 & $+/+x+1+$ & 1 & 7.00 & 7 & 0 & 0 & \\
\hline E12.5 & $\mathrm{Bst} /+x+/+$ & 3 & 8.67 & 26 & $23.08 \%(6)$ & $11.54 \%\{3\}$ & \\
\hline E12.5 & $+1+x+1+$ & 1 & 7.00 & 7 & 0 & 0 & \\
\hline E13.5 & $\mathrm{Bst} /+x+1+$ & 2 & 9.00 & 18 & $38.89 \%(7)$ & $27.78 \%\{5 \mid$ & \\
\hline E13.5 & $+1+x+1+$ & 2 & 8.00 & 16 & 0 & 0 & \\
\hline P0-adult & Bst $/+x+1+$ & 36 & 6.56 & 236 & $36.44 \%|89|$ & $8.05 \%(19)$ & $30.86 \%$ \\
\hline
\end{tabular}

${ }^{a}$ Numbers listed are animals analyzed, which are fewer than the total number of animals collected.

${ }^{b}$ At embryonic day 10.5 (E10.5), embryos are categorized by size: small embryos are defined as those less than $4 \mathrm{~mm}$ in crown to rump length. After E11.5, embryos are phenotyped as Bst/+ if they have short/twisted tails. Numbers in parentheses are actual counts.

${ }^{6}$ At E10.5, diminutive embryos are those that are less than $3.5 \mathrm{~mm}$ in length. Also included in this category are E11.5 or older embryos and neonates that are exencephalic. Together, these are considered to be more severe forms of the Bst/+ mutant phenotype. The high percentage of exencephaly in E13.5 litters is probably a reflection of the small sample size.

"Asymmetry is defined as greater than $25 \%$ difference in bilateral eye sizes in the E10.5 (Bst $/+)$ embryo and differential pupillary reflex in the Bst/+ adult. At older embryonic ages, asymmetry is difficult to measure using our sampling method. However, the variability in the state of fissure fusion suggests that retinas continue to develop asymmetrically in a subset of Bst/+ embryos.

presumed $B s t /+$ embryos in group 2 exhibit no atypical features compared to their larger (presumably $+/+$ ) littermates. However, the diminutive embryos in the third group exhibit features more appropriate for embryos that are up to 2 days younger than their littermates. Very likely, these severely under-developed embryos are in the process of being resorbed; TUNEL results confirm that most cells in these embryos are undergoing apoptosis. No such dramatic differences between littermates are seen in older litters. There are sporadic resorptions at this age in both $+/+\times+/+$ and $B s t /+\times+/+$ litters (averaging about 1 resorption per litter). The litter size ranges from 6 to 11 among $+/+x+/+$ controls (mean $=8.75 ; 4$ litters), and 8 to 10 among $B s t /+\times+/+$ litters (mean $=9 ; 5$ litters).

\subsubsection{E11.5.}

This is the earliest age at which $B s t /+$ embryos can be positively identified by their shortened-tail phenotype (Fig. 4.1). Bst/+ embryos consistently make up between 1/4 to 
$1 / 3$ of the litters from this point onward. The mean length of the Bst embryos $(5.9 \mathrm{~mm})$ is visibly less than that of the $+/+$ littermates $(6.3 \mathrm{~mm})$. Sporadic resorptions occur at about the same frequency as at E10.5. There is clear evidence of exencephaly in a subset of
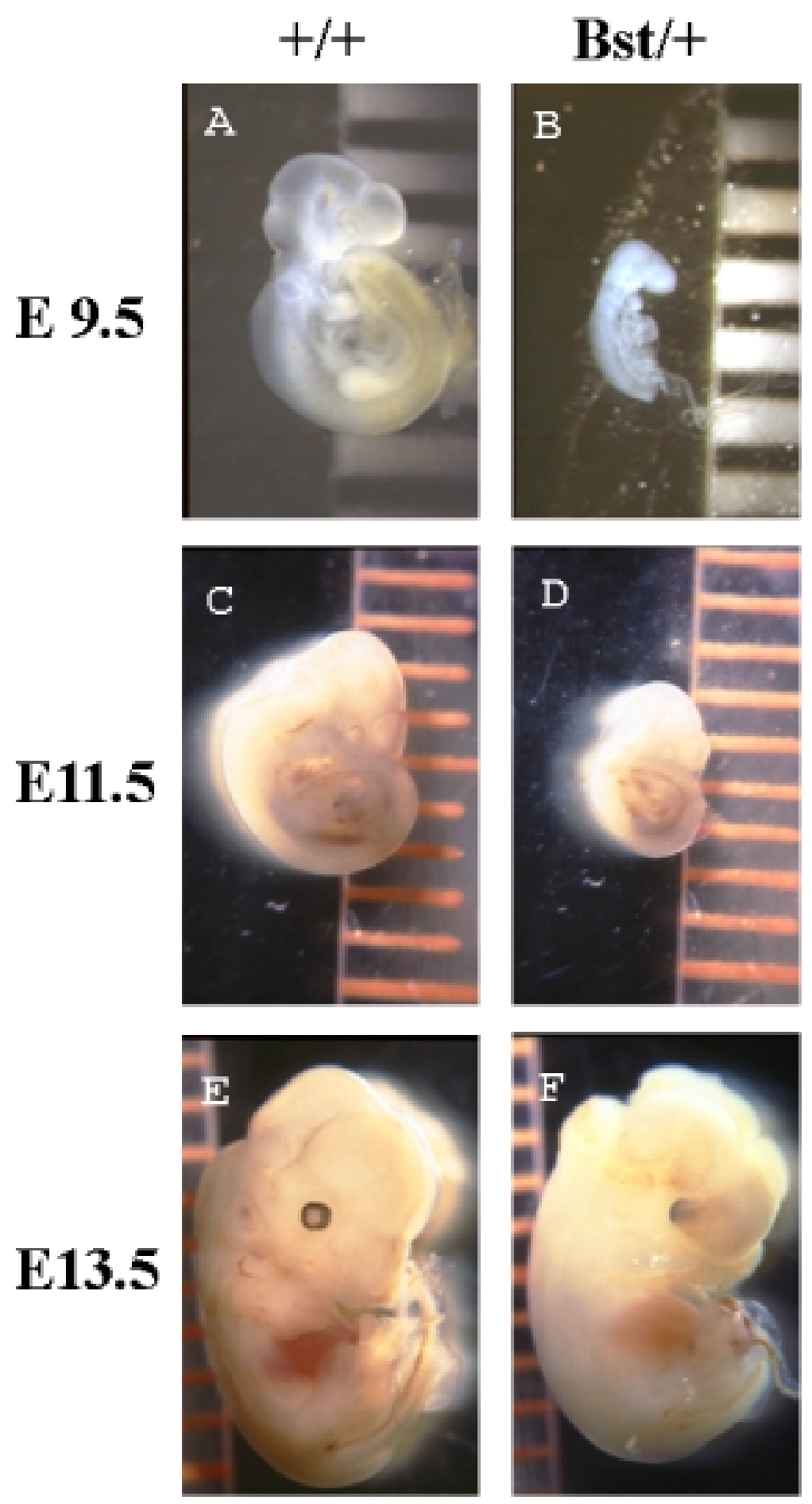

Fig. 4.1 Bst/+ embryos exhibit developmental defects. Top panels show E9.5 embryos. Middle panels show E11.5 embryos. Lower panels show E13.5 embryos. Embryos A, C, and $\mathrm{E}$ are wildtype. Embryos B, $\mathrm{D}, \mathrm{F}$ and $\mathrm{G}$ are mutants. The embryo in B represents an extreme phenotype; other presumed $B s t /+$ are larger, but not comparable to embryo in A. The size difference between the wildtype and $B s t /+$ is still quite evident at E11.5, when the tail length is just beginning to be measurable. The shorter tail is clearly identifiable in older embryos, such as in $\mathrm{G}$, when compared to that of the wildtype in E. Both F and G exhibit open anterior neuropore, a condition that gives rise to exencephaly. $G$ is also missing much of its midbrain, representing a more severe phenotype. The ruler in the background is graduated in $\mathrm{mm}$.

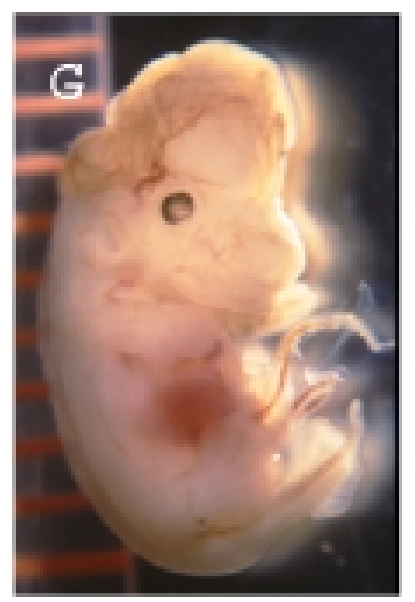


$B s t /+$ embryos. Within our sample, the proportion of exencephalic $B s t /+$ embryos is comparable to the proportion of exencephalic neonates (fewer than 1 affected per 2 litters on average, but as many as 2 per litter).

\subsubsection{E12.5.}

Litter size and resorption ratio from both $B s t /+\times+/+$ and $+/+\times+/+$ mating remain comparable to those at E11.5 (averaging 8 embryos and just over 1 resorption per litter in both groups). The average body length of $B s t /+$ embryos continues to trail that of the $+/+$ littermates $(8.1 \mathrm{~mm}, \mathrm{n}=7$, and $8.9 \mathrm{~mm}, \mathrm{n}=9$, respectively). The tail of $B s t /+$ embryos begins to show noticeable kinks. The exencephalic embryos in our sample have more pronounced expansion of the brain than those at E11.5 but would likely have survived until birth, as their other organs appear to develop normally.

\subsubsection{E13.5.}

Average litter size remains at 8 for both $B s t /+\times+/+$ and $+/+\times+/+$ litters. There are between 1 and 2 resorptions per litter. The size of $B s t /+$ embryos trails that of the $+/+$ littermates by the same margin as before $(9.8 \mathrm{~mm}, \mathrm{n}=3$, and $10.6 \mathrm{~mm}, \mathrm{n}=4$, respectively; Fig. 4.1). The development of digits can be observed at this time, and a fraction of Bst/+ embryos begin to manifest polydactyly, principally on the right rear paw. This is consistent with our observation among polydactylous Bst/+ adults (see Chapter 3), whose right rear paw nearly always exhibits a supernumerary and/or hypertrophied medial digit. Their left front paw is somewhat less vulnerable (60\% of affected cases) and exhibits only hypertrophy of the medial digit. Their left rear and right front limb extremities are almost never affected; each exhibiting anomaly in less than 5\% of the total cases .

\subsubsection{Retinal development.}

4.3.2.1 Bst/+ retinas are slightly undersized and fuse late during development.

In our observation, adult $B s t /+$ mice have relatively normal-appearing eyes that are only marginally smaller than those of the $+/+$, and none were out of proportion with the somewhat smaller girth of the $B s t /+$ mice in general. Our embryonic data are supportive of this observation. Of the $B s t /+$ animals we have examined thus far among both adults and embryos ( $n>200$ ), only a single still-born individual was found to be microphthalmic (both of its retinas were thick and heavily folded in the absence of vitreous cavities). 
At E9.5, optic vesicle invaginates to become optic cup. Lens formation is well under way. We have observed no grossly abnormal features in optic vesicles examined at this age. By E10.5, the depth of presumed +/+ optic cups (measured as ocular depth) exceeds that of presumed $B s t /+$ by an average of $60 \mu \mathrm{m}$. The same $60 \mu \mathrm{m}$ ocular length differential persists between groups through E13.5 (Fig. 4.2 A). However, since ocular length increases by nearly 4 fold between E10.5 and E13.5 (from $150 \mu \mathrm{m}$ to $550 \mu \mathrm{m}$ ), and given the relatively spherical shape of the eye during that period, the actual
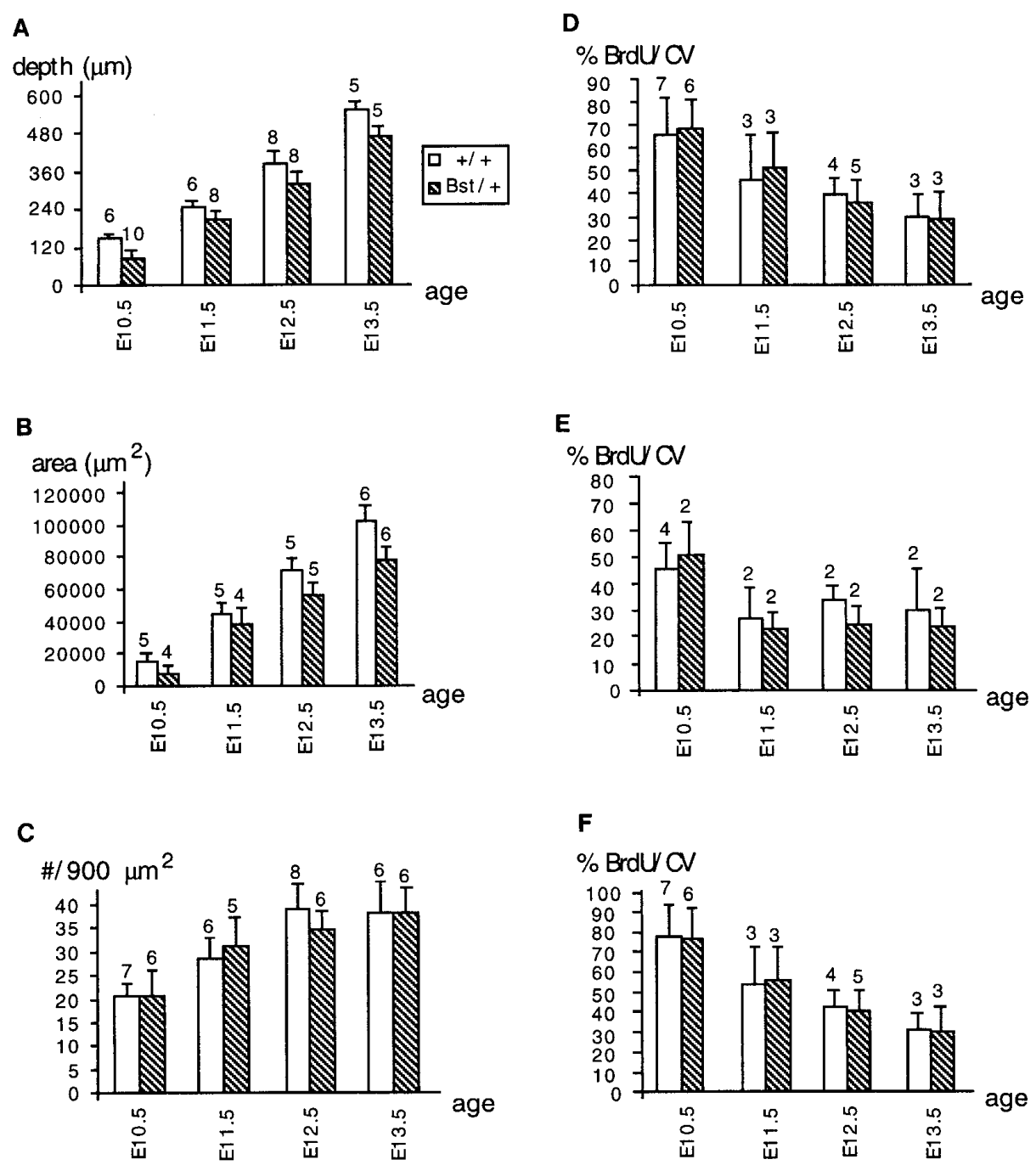

FIG. 4.2 Retinal growth and cellular proliferation in Bst/ + from E10.5 to E13.5. Histograms comparing size and proliferative indices of embryonic Bst/+ (open bars) and $+/+$ (shaded bars) retinas. Numerals above error bars indicate the number of animals in each case. (A) Histogram of ocular growth, as measured from iris to optic stalk. Bst $/+$ eyes are smaller than those of the $+/+$. The size deficit is maintained throughout the period between E10.5 and E13.5. (B) Histogram of retinal growth, represented by area of midretinal sections (see Materials and Methods). Mimicking the differential seen in ocular size between E10.5 and E13.5, the Bst/ + retina trails that of the $+/+$ in area by a relatively stable amount. (C) Histogram of retinal cell density from E10.5 through E13.5. There is no substantial difference between groups. (D) Histogram of proliferative index (PI) for the whole retina, measured as percentage of cells labeled by BrdU $1 \mathrm{~h}$ postinjection. The rate of decrease in PI mirrors that of the increase in cell density. The differences between groups are negligible during this growth period. ( $E$ and F) Histograms of PI in dorsal and ventral retinas, respectively. In both groups, PI in the dorsal retina is lower than that of the ventral retina at E10.5 and E1 1.5, but the disparity decreases with age and disappears by E13.5. The difference between groups is unremarkable. 
difference in ocular size between $B s t /+$ and $+/+$ is proportionally greater at E10.5 than at E13.5 (approximately 2:5 and 4:5, respectively). That is, a large difference in retinal size exists between $B s t /+$ and $+/+$ at E10.5, but their retinas grow at a similar rate thereafter, leading to a reduction in their proportional difference in older embryos.

The cross sectional area of the retinas shows the same gradual decrease in proportional difference between groups with age progression (Fig. 4.2 B). At E10.5, the ratio between the areas of equivalent $B s t /+$ and $+/+$ retinal cross sections is about 1:2. By E13.5, it has become 7:10.

The most noticeable difference between $B s t /+$ and $+/+$ embryos is that in the mutant, optic fissure closure takes place later than in the wild type and requires more time to complete. At E11.5, wild type retinas have largely reached the point at which fissure margins have come into contact and begun to fuse. The fusion process starts in the proximal retina, close to the optic stalk, and progresses distally. In contrast, most E11.5 $B s t /+$ retinas are wide open along their ventral aspect; very few of them exhibit any contact between the fissure margins. By E12.5, all $+/+$ retinas have at least partially-fused optic fissures (Fig. 4.3), while only a quarter of $B s t /+$ retinas show signs of any fusion; many continue to manifest open gaps between fissure margins. In some cases where fissure margins have made contact, obvious distortions in the neural retina are present. By E13.5, optic fissure fusion is complete in the $+/+$ (Fig. $4.3 \mathrm{D}$ ); whereas less than half of the $B s t /+$ retinas are fused (Fig. $4.3 \mathrm{E}$ ), with some showing persistent gaps between fissure margins (Fig. $4.3 \mathrm{~F}$ ). The symptomatic buckling of the neural retina near the optic fissure first seen in some E12.5 Bst/+ embryos has also become more pronounced at this age. In remarkable contrast, fusion appears to occur without delay in the pigmented retina after the opposing margins have made contact (Fig. 4.3 E).

\subsubsection{Bst/+ retinas have normal cell density.}

Between E10.5 to E13.5, the density of retinoblasts is comparable between the retinas of mutant and control animals (Fig. 4.2 C). Given the smaller size of Bst/+ eyes however, the total number of retinoblasts in the $B s t /+$ retina is considerably less than that of the equivalent $+/+$ retina. This is especially true in the younger embryos, where dimensional differences between the $B s t /+$ and $+/+$ retinas are proportionally greater than those at later embryonic ages. Given the similarity in cell density, the differences in total retinal cell numbers between groups are effectively reflected by the changes in retinal dimension, such that at E10.5, there are more than 3 times as many cells in the $+/+$ retina as there are in the $B s t /+$ littermate; whereas by E12.5, there are less than twice as many 

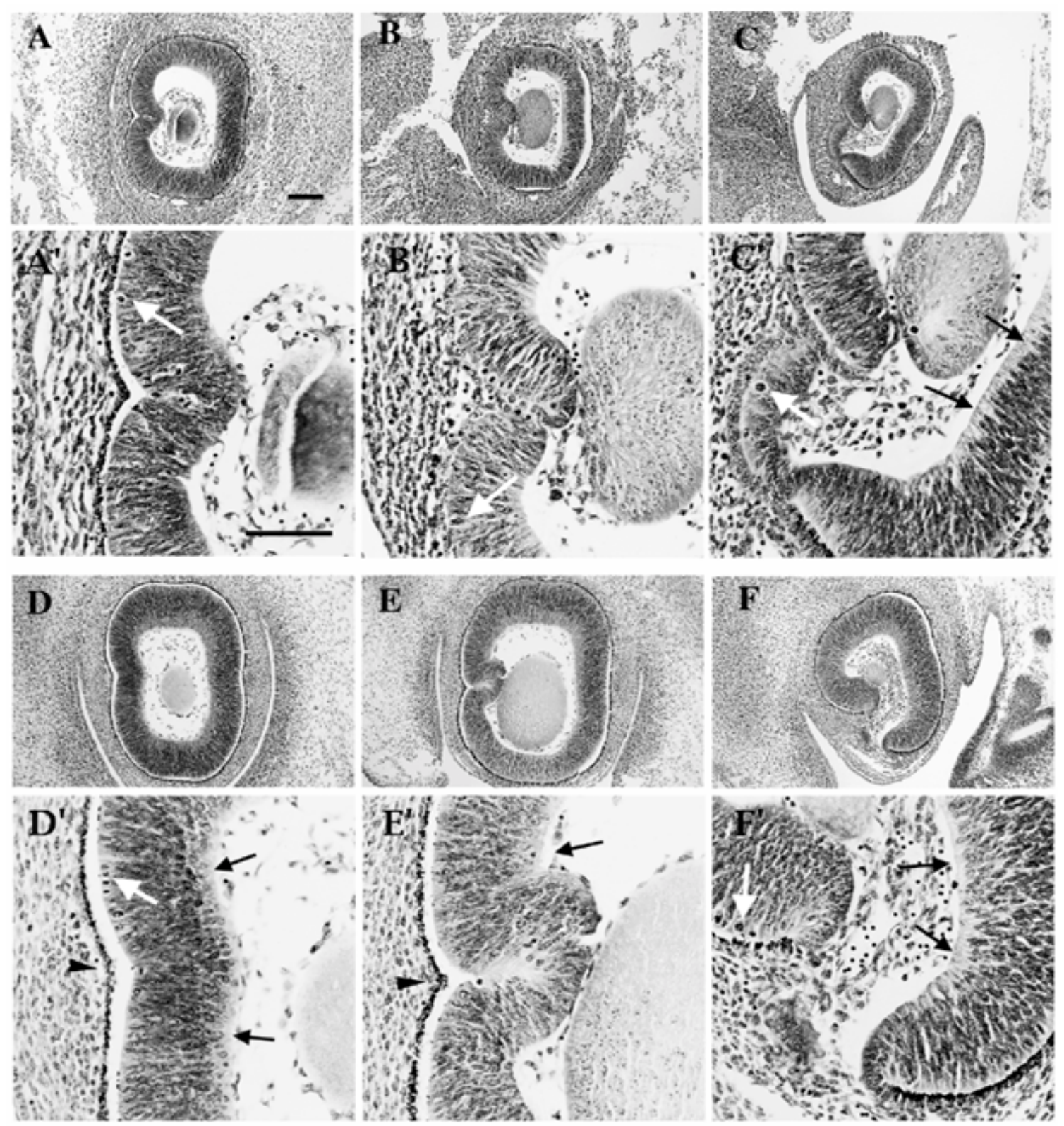

Fig. 4.3 Optic fissure fusion in the developing $B s t /+$ retina. Representative retinal sections of $+/+$ and $B s t /+$ embryos taken at E12.5 (A, B, C) and E13.5 (D, E, F). The sections are obtained from equivalent positions in the respective eyes. A' through $\mathrm{F}^{\prime}$ are higher magnification views of the optic fissure region in A through $\mathrm{F}$. White arrows mark mitotic figures. Dark arrows indicate the acellular layer in the vitreal aspect of the retina. A) and A') Retina of a typical +/+ embryo at E12.5, showing fusion of the optic fissure. B) and B') Mildly symptomatic retina of an E12.5 $B s t /+$ embryo showing contact between the fissure margins but no apparent fusion. C) and C') Severely symptomatic retina of another E12.5 Bst/+ embryo exhibiting retinal distortions. Both Bst/+ eyes are marginally smaller than that of the $+/+$. The thickness of the neural retina, the appearance of the dorsal acellular region, and the number of mitotic figures are comparable between groups. The same differences and similarities are seen in E13.5 retinas. D) and D') Typical E13.5 +/+ retina showing fully fused optic fissure. E) and E') Mildly symptomatic E13.5 Bst/+ retina, where the optic fissure appears to have fused, yet there is considerable distortion, suggesting that some growth of the retina has continued beyond the normal stopping point. F) and F') Severely symptomatic E13.5 Bst/+ retina, where optic fissure margins have yet to make contact. The overall rate of growth appears comparable in the three E13.5 retinas. The acellular zone has progressed ventrally, and the expanded euchromatic cell layer is visible immediately beneath it. Note the similarities between groups. The pigmented retina appears to fuse normally in the $B s t /+$ and does not follow the abnormal growth in the neural retina (arrowheads). Scale bars represent $100 \mu \mathrm{m}$. 
cells in the same $+/+$ retina. This indicates that overall retinal growth is not impeded in the $B s t /+$ during later development in spite of earlier deficits. There are other indications that this may be the case. For instance, from E11.5 onward, in both $B s t /+$ and +/+ retinas, there is an emergence of a sparsely cellularized layer on the vitreal surface of the dorsal retina through which ganglion cell axons will eventually course (Watanabe et al., 1991). This non cellularized (i.e., without cell nuclei) zone progresses ventrally and covers the entire retina by E13.5. At this age, in both $B s t /+$ and $+/+$, the euchromatic layer of post migratory and differentiating retinal ganglion cells and INL cells are clearly visible close to the vitreal surface (Fig. 4.3 D, E, F).

\subsubsection{Cellular proliferation rate is normal in the $B s t /+$.}

In both $B s t /+$ and $+/+$ retinas, the proliferative index (PI) is highest at E10.5 (nearly 70\%) and gradually drops to about 30\% at E13.5 (Fig. 4.2 D). This is consistent with findings reported by others for mice and rats of similar developmental age (Konyukhov and Sazhina, 1975; Silver, 1976; Burmeister et al., 1996; Alexiades and Cepko, 1996). Overall, there is no detectable difference between the average PI of Bst/+ and $+/+$ at any of the ages examined (Fig. 4.4). We have also looked for PI differences between dorsal and ventral retinas (Fig. 4.2 E, F). In both Bst/+ and +/+ retinas, a small strip of the dorsal retina opposite the optic fissure has a noticeably lower PI than the rest of the retina at E10.5 and E11.5. This regional difference is much less noticeable at E12.5 and disappears by E13.5. There may be a correlation between the lower PI in the dorsal retina and the spatial sequence of cellular differentiation, given our observation that at E11.5 and E12.5, the dorsal retina has more euchromatic (presumably post-mitotic) cells than the rest of the retina. We have found no difference between the PI of proximal and distal regions of the retina.

\subsubsection{Cell death is normal in the Bst/+.}

Cell death in the neural retina is most apparent between E11.5 and E12.5. During that period, pyknotic figures are observed in both $B s t /+$ and $+/+$ retinas, mostly in the ventral region adjacent to the optic fissure (Fig. $4.5 \mathrm{~A}, \mathrm{~B}$ ), in a pattern appropriate for the developmental age (Silver and Hughes, 1973, Martín-Partido et al., 1988). More than half a dozen pyknotic figures could be seen in each E12.5 section. This is similar to the number of TUNEL-labeled cells in equivalent sections (Fig. $4.5 \mathrm{C}$, D). The only other region where cell death is occasionally seen is in the proximal dorsal retina, coinciding 


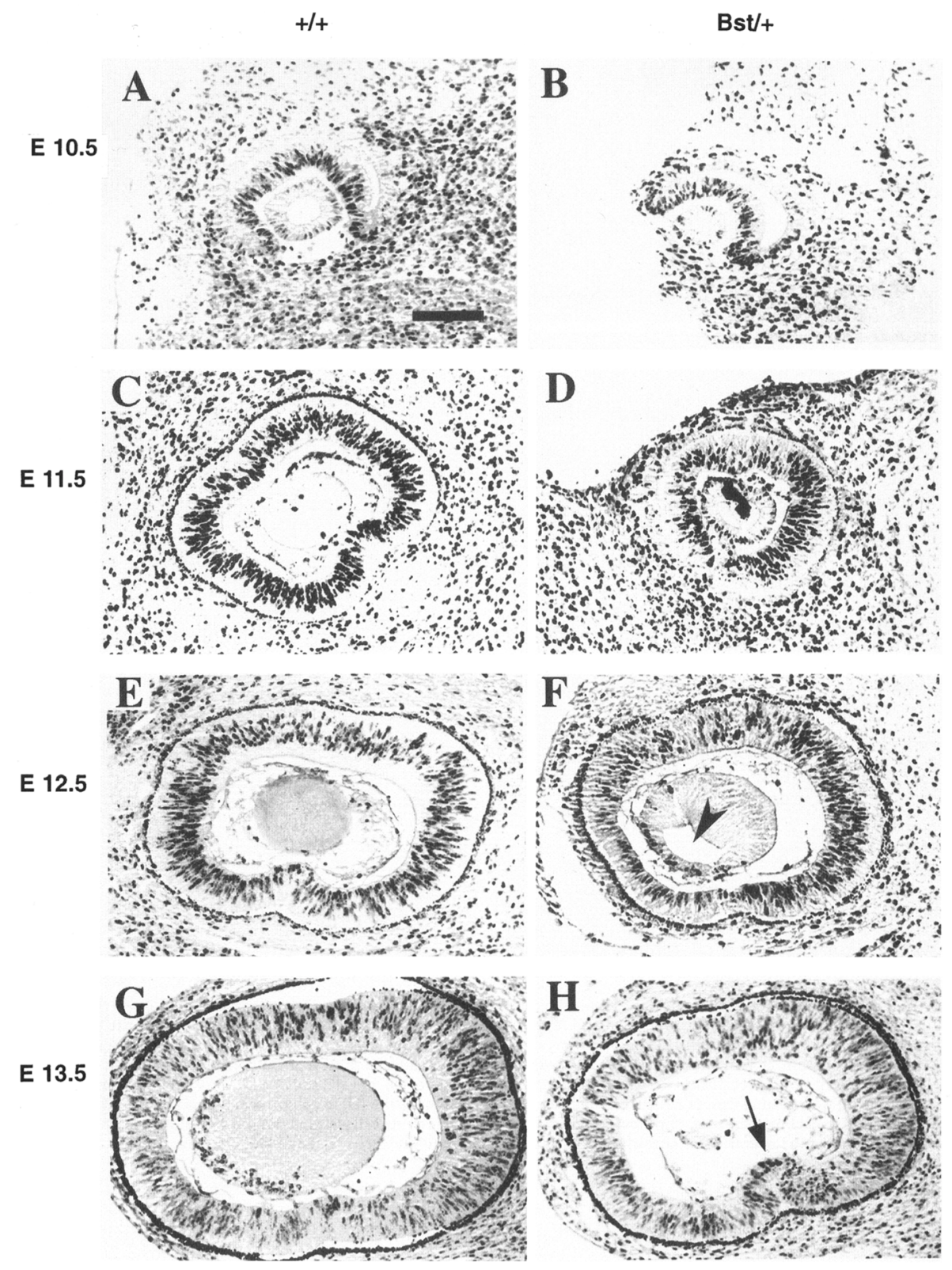

FIG.4.4 Retinal growth and cellular proliferation profiles. Representative photomicrographs showing E10.5 (A, B), E11.5 (C, D), E12.5 (E, $\mathrm{F})$, and E13.5 $(\mathrm{G}, \mathrm{H})$ retinas from $+/+$ (left column) and Bst/+ (right column) embryos immunostained for BrdU labeling. (A and B) At E10.5, the $B s t /+$ retina is thinner and smaller, but no less proliferative than its $+1+$ counterpart. That is, the numeric proportion between the dark, BrdU-labeled cell nuclei seen in the retinoblastic layer and the cell bodies seen in the adjacent CV-stained retinal section (proliferative index, or PI; Fig. 1D) is comparable between groups. (C and D) At E11.5, the $+1+$ retina has already begun to fuse, while that of the Bst/+ has not. Proliferative index remains comparable betwcen the two groups. (E and F) At E12.5, PI is again similar between the $+/+$ and the $B s t /+$ retinas. The small differences in label density seen here are typically regional and are not sustained through consecutive sections. Note that the lens cavity, which has nearly disappeared in the $+/+$ by this age, remains substantially visible in the Bst $/+$ (arrowhead), indicating that lens development is delayed to a certain degree in the $B s t /+.(\mathrm{G}$ and $\mathrm{H})$ In E13.5 retinas, the $B s t /+$ retina continues to be smaller than its $+1+$ counterpart. There is no difference in proliferative index between groups. Note distortion of the retina at the Bst/+ optic fissure (arrow). Scale bar represents $100 \mu \mathrm{m}$. 

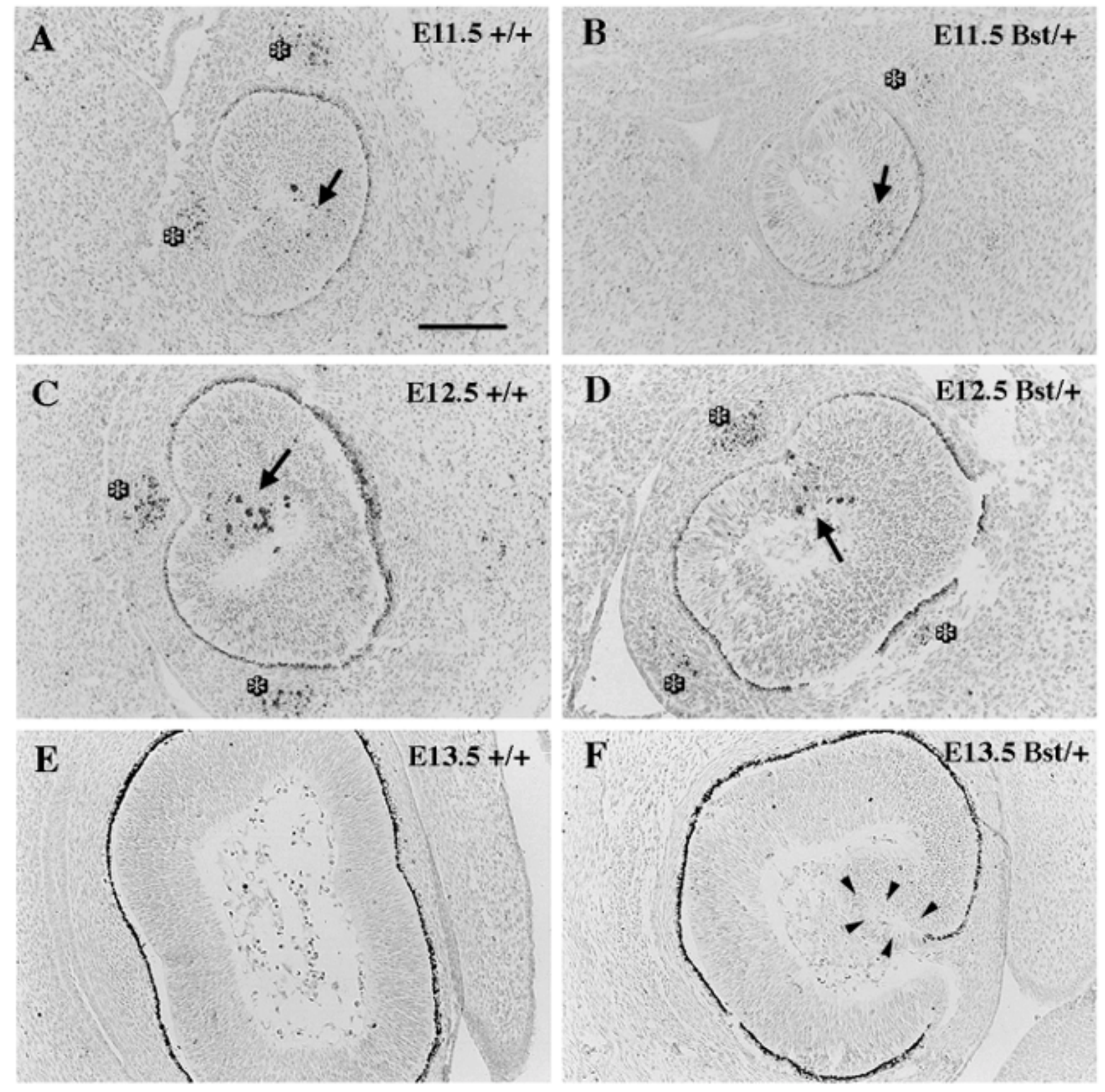

Fig. 4.5 Cell death in the developing $B s t /+$ retina. TUNEL-stained retinas from +/+ (A, C, E) are compared to Bst/+ $(\mathrm{B}, \mathrm{D}, \mathrm{F})$ embryos from E11.5 through E13.5. A) and B) Wild type and Bst/+ E11.5 retinas. Arrows mark TUNELstained dying cells in the retina. Asterisks mark dying cells outside the retina. In the E11.5 +/+ embryo (A), TUNELstained cells are found in both ventral and dorsal aspects of the retina near the optic fissure, as well as at positions which will give rise to extra-ocular muscle attachment sites. The position and quantity of TUNEL-positive cells in the E11.5 Bst/+ retina (B) are comparable to those of the $+/+$. C) and D) E12.5 +/+ and Bst/+ retinas. Extensive TUNEL-positive cells are seen at the optic fissure in both the $+/+(\mathrm{C})$ and the $B s t /+(\mathrm{D})$ in comparable numbers. The number of labeled cells begins to decline thereafter, until there are very few identifiable dying cells left in both the $+/+$ and $B s t /+$ at E13.5, E) and F). Note the extensive folding of the neural retina (arrowheads) at the optic fissure in the $B s t /+(F)$. Scale bar represents $100 \mu \mathrm{m}$. 
with the area that has lower than average PI in younger retinas. By E13.5, pyknotic figures or TUNEL-labeled cells are seldom observed in the retina (fewer than 2 per section, Fig. 4.5 E, F). There are no obvious differences between $B s t /+$ and $+/+$ in the number of dying cells and their positions within the retina.

4.3.2.5 The exit of cells from the cell cycle is delayed in the Bst/+ retina.

The pattern and density of BrdU-labeled cells in P0 animals dosed on different embryonic days offer a glimpse of the sequential order within which cells become postmitotic. BrdU-positive cells are seen in the retinal ganglion cell layer and the superficial and deep retinoblastic layer of $+/+$ mice dosed on E10.5 (Fig. 4.6 A), indicating that retinal ganglion cells, cones, and amacrine/horizontal cells have begun to exit the mitotic cycle at this age. We estimate that 1,500 cells in the $\mathrm{P} 0+/+$ retina are heavily labeled with BrdU following an injection at E10.5. In contrast, there are few BrdU-labeled cells in the $B s t /+$ P0 retina dosed on the same day, and the number of intensely labeled cells (i.e., those cells born at the time of BrdU injection) is negligible, indicating that there are far fewer cells exiting the mitotic cycle in the $B s t /+$ retina than in the $+/+$ retina at E10.5 (Fig. $4.6 \mathrm{~B}$ ). It is only in E11.5-dosed Bst/+ retinas that we begin to observe labeled cells in a pattern and density that approximate those seen in E10.5-dosed +/+ retinas (Fig. 4.6 C, D). The difference remains evident in E12.5-dosed retinas (Fig. 4.6 E, F), where fewer heavily labeled cells are observed outside the retinal ganglion cell layer in the $B s t /+$ retina compared to that of the $+/+$. Numerically, there are about twice as many intensely BrdUlabeled cells in the E12.5-dosed $\mathrm{P} 0+/+$ retina than in the $B s t /+$ retina (approximately 4000 vs. 2000 cells). However, when taken into account the total number of cells in the retina at E12.5, the actual percentage of cells born (birth index) in $+/+$ and $B s t /+$ retinas are quite similar (4\% and 3\%, respectively, Fig. $4.6 \mathrm{G}$ ).

4.3.2.6 Retinal development is asymmetrically disturbed in the $B s t /+$ mutant.

Given the prevalence of asymmetry in optic nerve hypoplasia among $B s t /+$ adults (Rice et al., 1997), we were curious to see if there were any correlates of this phenotype in developing embryos. Bilateral differences are visible but not extreme, such that if a $B s t /+$ embryo exhibits anomalies in one eye, the other eye is invariably affected as well. Asymmetry is measurable early on. At E10.5, there are clear size disparities between the eyes in about $25 \%$ of $B s t /+$ embryos, which approximates the ratio of individuals among $B s t /+$ adults exhibiting asymmetric eye defects (Table 4.1). Unlike the polydactyly 
Fig. 4.6 Delayed neuronogenesis in the $B s t /+$ retina. BrdU-immunolabeled retinas from postnatal day $0(\mathrm{P} 0)+/+(\mathrm{A}, \mathrm{E})$ and $B s t /+(\mathrm{B}, \mathrm{C}, \mathrm{D}, \mathrm{F})$ mice, following BrdU injection at E10.5 (A, B), E11.5 (C, D), and E12.5 (E, F). Histogram of the total number of cells present in the E12.5 $+/+$ and $B s t /+$ retinas and the number of cells born $(\mathrm{G})$. Arrows mark BrdU-positive cells in the vitreal aspect of the retina (predominantly retinal ganglion cells). Black arrowheads mark BrdUlabeled cells in the future inner nuclear layer of the retina. White arrowheads mark labeled photoreceptors. A) In E10.5-dosed P0 +/+ retina, the retinal ganglion cell layer is distinctly labeled, confirming that retinal ganglion cells are born at E10.5. Labeled cells in the future inner nuclear layer and photoreceptor layer indicate that other cell types are also being born at that age, such as amacrine and horizontal cells. B) In contrast, P0 Bst/+ dosed on E10.5 shows very few BrdU positive cells in its retina; thus a considerably smaller number of cells, if any, have exited the mitotic cycle in the $B s t /+$ compared to the $+/+$ at this age. C) and D) A range of BrdU labeling patterns is seen in E11.5-dosed P0 Bst/+ retinas. The same variation is seen in $\mathrm{Bst} /+$ retinas dosed at different embryonic ages. None of the E11.5-dosed Bst/+ retinas are comparable to E10.5-dosed +/+ retinas in the level of BrdU labeling (see A). Gray arrowhead points to axons coursing through the optic nerve head, indicating that the delayed cell birth did not prevent at least some of the ganglion cell axons from finding the correct path in this animal. E) In P0 +/+ mice dosed on E12.5, the retinal ganglion cell layer, inner nuclear layer, and photoreceptor layer are heavily BrdU-positive. F) In this E12.5-dosed P0 Bst/+ retina, the extent of labeling is barely comparable to that of the $+/+$ retina dosed on E10.5 (see A). G) Cells born at E12.5. While the number of cells born in the $+/+$ is more than twice the number born in the Bst/+ (4500 vs 2000), the actual cell birth index (BI) is $5 \%$ in the $+/+$ and $4 \%$ in the Bst/ + once the total number of cells in the embryonic retina is taken into account. This partially explains the lack of differences in the proliferative indices (see Fig. 4.2). The number of animals examined is indicated above error bars. Variations in the thickness of retinal cross-sections shown here are due to differences in the angle of section. Scale bar represents $100 \mu \mathrm{m}$. 


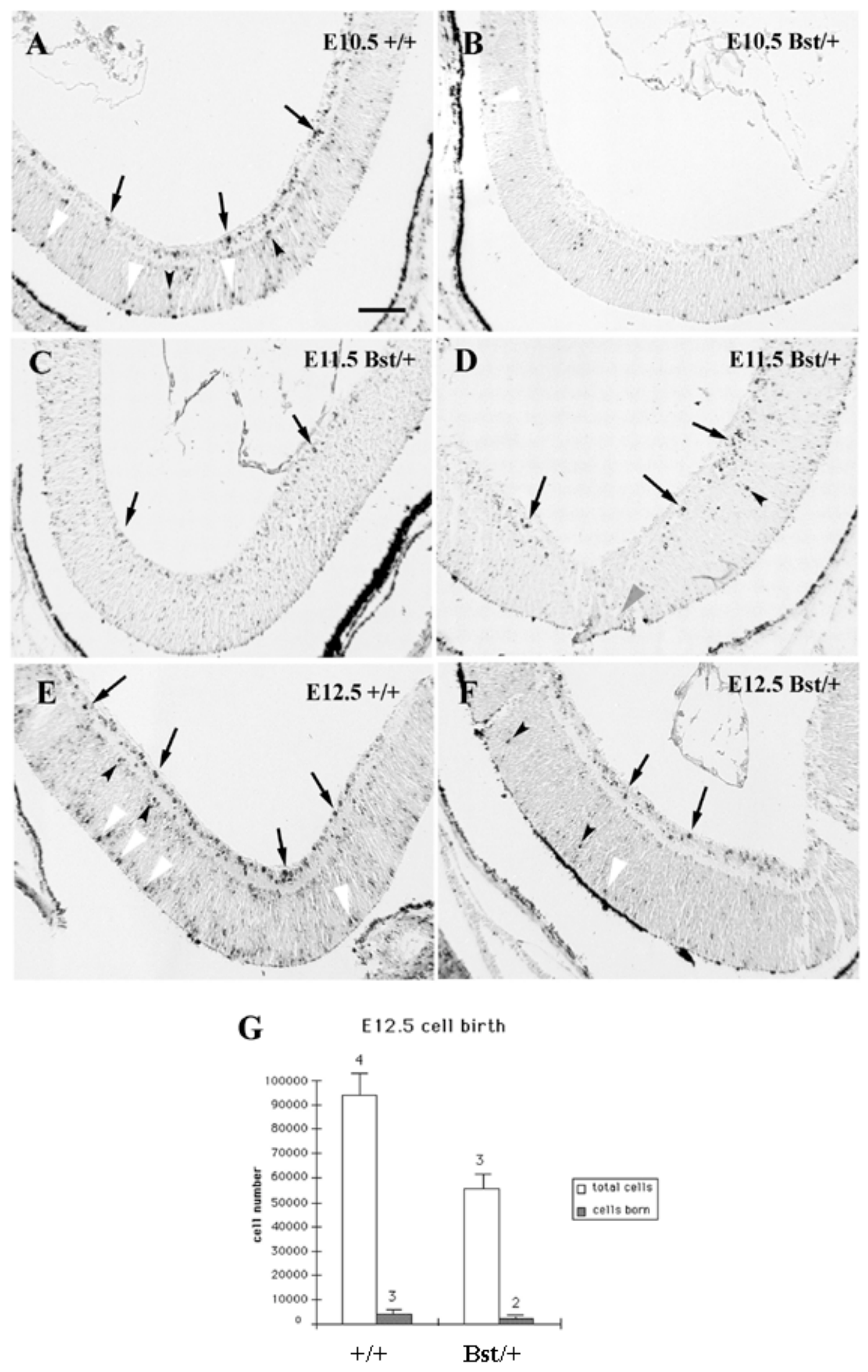


phenotype of the $B s t /+$, where asymmetry is the norm rather than the exception, and side preference is very predictable, both eyes in the $B s t /+$ mouse seem equally susceptible to developmental defects.

\subsection{Discussion}

One of the hallmarks of the Bst mutant retina is a failure in fissure fusion (Rice et $a l ., 1997)$. In this study we have sought to determine whether this developmental abnormality is the primary target of the mutation or whether it is the consequence of an earlier defect. In order to examine this issue, we studied embryonic Bst/+ retinas with special attention to developmental events that are thought to impact fissure fusion, such as cellular proliferation, differentiation and death. Perturbation of these events has been linked to other murine mutations affecting the fusion of the embryonic fissure, namely microphthalmia (mi; Hero et al., 1991), ocular retardation (or; Theiler et al., 1976), and the mouse knock-out of hairy and enhancer of split homolog-1 (Hes1; Tomita et al., 1996). Our results indicate that while the Bst mutation generates fissure fusion anomalies reminiscent of those seen in $m i$, and delayed retinal growth similar to what has been described in $o r$, the etiology of $B s t$ defects clearly follows a path distinct from those of the other mutations. In $B s t /+$, there is no abnormal proliferation or pyknosis of retinoblasts as in or; nor excessive growth of the pigmented retina as in mi. However, the manner in which fissure fusion defects occur in the $B s t /+$ embryos bears close resemblance to that of hemizygous Hes 1 -null mice, in which the developing mutant eye tends to be marginally smaller than that of the wild type and where fissure fusion occurs later than normal (Tomita et al., 1996). In the Hesl-null mouse, it has been shown that premature cellular differentiation is likely responsible for the retinal abnormalities. In this study we find that a delay in cellular differentiation could play a crucial role in causing similar retinal defects in the $B s t /+$.

4.4.1 Bst plays a role during early retinal development, independent of cell proliferation and cell death.

The fact that Bst/+ eyes are smaller than those of normal littermates at E10.5 (and likely by as early as E9.5) indicates that the mutant gene has a very early effect upon retinal development. In this context, the lack of proliferative differences between Bst/+ and $+/+$ retinas is significant. It is in contrast with two other mutations that affect fissure 
fusion: ocular retardation (or, attributed to a defect in the Chxl0 gene) and microphthalmia ( $m i$, caused by a mutation in the Mitf gene). In or mutants, the cell cycle is lengthened in a population of cells in the distal retina; which may account for delayed retinal growth and ultimately produce structural anomalies (Robb et al., 1978; Osipov and Vakhrusheva, 1981; Liu et al., 1994; Burmeister et al., 1996). In mi mutants, there is an abnormal proliferation of pigmented retinal cells at the optic fissure that may prevent fissure fusion and contribute to the eventual collapse of the vitreal cavity (Hero, 1990). Neither of these symptoms are found in Bst/+ retinas. Alternatively, premature or excessive cell death are processes that have been linked to retinal hypoplasia, in a manner typified by the untimely and ectopic cell death described in or mutants (Theiler et al., 1976; Robb et al., 1978). There is no indication, however, that apoptosis is disturbed in $B s t /+$ during arguably the most functionally critical time of retinal formation -- the period immediately preceding the exit of retinal ganglion cell axons into the optic stalk (Silver and Hughes, 1973). Our data point to the conclusion that Bst has an effect on the number of neuroblasts available for growth in the optic vesicle; which would indirectly affect optic fissure fusion. However, this effect is unlikely to be achieved through the control of the rate of cell proliferation or cell death.

\subsubsection{Bst may regulate cell differentiation.}

Among our observations, a very telling clue for Bst gene action lies in the apparent delay in cellular differentiation in the $B s t /+$ neural retina. We found that in $B s t /+$ mice, retinal cells are born noticeably later than in $+/+$ littermates, sometimes by as many as 48 hours, suggesting that $B s t /+$ retinoblasts are likely to remain mitotically active several cell divisions beyond their wildtype counterparts. While this observation may appear to conflict with the absence of differences in the proliferative index between groups, it is evident that the proportion of cells exiting the cell cycle is not greatly different between groups at E12.5 (Fig. 5G). In essence, comparing the retinas of $+/+$ and $B s t /+$ littermates is akin to comparing $+/+$ retinas from different embryonic ages; the number of cells exiting the cell cycle would be clearly different between groups based upon wellcharacterized spurts of retinogenesis, while their proliferative indices undergo relatively little change between E11.5 and E13.5 (see Fig. 1D). A similar situation exists in the or mutant, where cellular differentiation and retinal growth are known to be delayed throughout the eye, yet the measured proliferative index is largely normal but for a limited region of the peripheral retina (Osipov and Vakhrusheva, 1981; Burmeister et al., 1996; Mark Hankin, personal communication). 
It is of interest to compare the above results with the hemizygous Hes1-null mouse phenotype (Ishibashi et al., 1994, Tomita et al., 1996). Hesl is believed to be an inhibitory regulator of neurogenesis and may play a key role in the Notch signaling pathway (Artavanis-Tsakonas et al., 1995, Austin et al., 1995). Inactivation of Hesl leads to premature cellular differentiation, while overexpression of Hes 1 maintains neuroblasts in the mitotic state. Hesl-null mice exhibit numerous retinal and skeletal phenotypes quite similar to those of the $B s t /+$, including fissure fusion defects. These similarities suggest that $B s t$ likely plays a role in the same morphogenetic pathway as does Hesl. Given that the mutant allele of $B s t$ appears to inhibit rather than promote cellular differentiation, $B s t$ and Hes 1 could conceivably act as antagonist regulators of early neuronal differentiation in the retina. Regulatory interactions of this nature may be an efficient means to control developmental events characterized by critical temporal or spatial coordinates. Examples of these can be found in mechanisms underlying morphogenetic processes as local as cell adhesion and motility and as global as body axis determination, suggesting that they are evolutionarily relevant and more ubiquitous than currently documented or hypothesized (Alevizopoulos and Mermod, 1996; Kerszberg, 1996). If Bst indeed acts in conjunction with Hes I to regulate cellular differentiation, it would likely be a member of the proneural gene family, which as a group potentiates neuronal precursors towards their eventual fate. Proneural genes work in coordination with boundary genes, such as members of the Pax family, which are thought to specify cell types at defined positions (for reviews, see Chalepakis et al., 1993, MacDonald and Wilson, 1996, Bang and Goulding, 1996). Proper neuronal differentiation is dependent upon well-timed interactions between both proneural and boundary genes. We have seen evidence of ectopic Pax2 expression in the Bst embryonic retina, which suggests a loss of boundary cues, possibly as a consequence of delayed cellular differentiation (see Chapter 5).

4.4.3 The Bst mutation likely disrupts timing and coordination of developmental events during retinal morphogenesis.

It is important to consider that $B s t /+$ embryos are smaller than their $+/+$ littermates by as early as E9.5-E10.5, before any significant neurogenesis has taken place in the retina. At that time, and throughout the rest of embryonic growth, the ocular and whole-embryo size deficit is maintained but not exacerbated in $B s t /+$, indicating that the underlying growth program is unaffected by the perturbations that are responsible for the early developmental deficit. It is possible that the $B s t$ mutation causes a general delay in growth prior to retinal morphogenesis; the defects observed in the neural retina may be a 
byproduct of this earlier, more global event. Interestingly, two defects seen in the Bst/+ mutant mouse - exencephaly and polydactyly - are also characteristic of other mutations in which retinal development is disturbed, such as Hesl-null (Tomita et al., 1996) and fidget mutant mice ( $f i$; Grüneberg, 1943). This indicates that $B s t$ may be one of a number of molecules shared by regulatory pathways guiding the development of these organs. Without question, the diminished stature of $B s t /+$ mice and their multiple-organ defects indicate that the role of $B s t$ is wide-ranging. However, our observations suggest that within the affected organs, not every tissue or cell type is equally sensitive to the Bst mutation, given that most of these organs only exhibit partial defects, and frequently no defect at all. It is particularly noteworthy that the pigmented retina, which derives from the same neural epithelium as the neural retina, appears unaffected by the Bst mutation (the development of the PE is likely guided by its own genetic determinants, as evidenced by mutations in which the pigmented retina is selectively affected; see Hero, 1989; Sidman et al., 1996). Moreover, it is possible that within the affected tissues, only a subclass of cells is critically sensitive to the expression of $B s t$, and perhaps such sensitivity is limited to a certain temporal window dictated by the interactions between regulatory molecules. Like the Pax genes (McDonald and Wilson, 1996), Bst may be expressed at different times during development, and not necessarily within the same tissues, to regulate different aspects of organogenesis. The conspicuous sensitivity of the neural retina to the $B s t$ mutation could be attributed to the fact that, perhaps more so than with other structures, morphogenesis of a functional retina is dependent upon tightly orchestrated cellular interactions within narrow temporal windows that define the guidance and exit of retinal ganglion cell axons from the retina.

The hypothesis above also allows us to address the variability witnessed in the $B s t /+$ phenotype. Embryonic development progresses at different rates in the Bst/+ and $+/+$ mice. In the eye, the extent of fissure fusion is a source of considerable variation among Bst/+ embryos after E11.5, ranging from normal to much delayed (Fig. 4.3). To a lesser degree, the variability is also present within $B s t /+$ individuals in the form of asymmetric eye development in the embryo and differential retinal hypoplasia in the adult. The extent and proportion of asymmetry in the embryonic mutants are similar to those observed in the adult population (Table 4.1). Conceivably, the range of defects we are witnessing during fissure fusion could be the consequence of subtle differences in the timing of the engendering developmental events, which can variably alter the fate of the developing tissue depending on the extent of the synchrony. 
Chapter 5. Bst is a regulator of early retinal development: evidence of interaction between Bst, Pax2 and Hes1.

\subsection{Introduction.}

Eye development, as a readily observable organogenetic event, is dependent upon the timely expression of regulatory molecules and the orchestrated interactions between such molecules. Functionally, the regulatory molecules may be grouped into three principal classes. At the very core, there is a group of molecules which maintains the cellular clockwork, principally in the form of cyclins, cyclin-dependent kinases (cdk) and associated factors that control the onset and duration of various aspects of the cell cycle (Pines, 1994a,b; Morgan 1995; Edgar and Lehner, 1996; King and Cidlowski, 1998; Studzinski and Harrison, 1999). In close functional association with the cell cycle maintenance molecules are a second group of molecules which either promote or inhibit cellular transformations, such as differentiation and programmed cell death, which are the driving forces behind morphogenesis. These latter are typically DNA-binding molecules, such as $p 53, M y c$, sina, and a number of molecules containing the helix-loop-helix motif which directly target the expression of other genes (Kageyama et al., 1995; Fotebar et al., 1996; Kang et al., 1998; Dickson, 1998; Fulci and Van Meir, 1999; Cole and MacMahon, 1999, see also Chapter 2). Finally, the direction and shape of the transformation are guided by a third class of molecules communicating positional cues needed for establishing orientation and boundaries, such as members of the gap, pair rule, segment polarity and homeotic gene families, namely Hox and Pax, and numerous other homeobox-containing genes like Wnt, Otx, etc. (Chalepakis et al., 1992; Dahl et al., 1997; Gellon and McGinnis, 1998) acting through cell cell interactions gated by molecules such as Notch (Artavanis-Tsakonas, 1995). The complexity and close functional tolerance of the retinal anatomy behoove the involvement of large numbers of all three types of molecules during its genesis. However, the well-characterized order of retinal development, combined with an increasing number of genes and loci known to have specific effects within the developing eye, also make the retina an excellent structure for studying the interactions between the regulatory molecules. As new genes and loci are discovered to play a role in eye development, it is both helpful and necessary to characterize their role within the context of an interactive network of molecules comprised of those with more clearly identified functions. 
We have examined embryonic development in the belly spot and tail (Bst) mutant mouse, and found that the atrophy of the retina and the optic nerve in the adult mutant mouse may be caused by abnormal embryonic development (Rice et al., 1997; Tang et al., 1999; see Chapter 4). We observed a delay in cell birth in the embryonic mutant retina, suggesting that the Bst mutation is an early-expressed gene which regulates cellular differentiation. In these studies, we examined the effects of the Bst mutation in relation to two other molecules known to play significant roles during the same developmental period, and whose mutant or knockout retinal phenotypes exhibit intriguing similarities with that of the Bst/+ mutant - the boundary gene Pax2, and the neurogenic gene Hes1. We studied eye development in compound heterozygous mutants involving a combination of Bst with Pax2 or Hes1. The use of compound heterozygous mutants is a powerful tool for detecting interactive relationships between genes. It has been used extensively in Drosophila studies (Wilson and Jacobson, 1977; Gorman et al., 1992; de la Pompa, 1994; Pignoni et al., 1997) In the mouse, the technique has been successfully employed to delineate the synergistic effect between fidget ( $f i$ ) and ocular retardation (or) during eye development (Konyukhov and Sazhina, 1975; Osipov and Vakhrusheva, 1981), as well as the interaction between Pax2 and Pax5 during brain development (Urbanek et al., 1997).

The Pax 2 protein is present in the optic vesicle at E9.0; its domain of expression becomes gradually restricted in the ventral aspect of the optic cup as the optic fissure is formed. By E11.5-12.5, its expression is further restricted to within only the optic disc and optic stalk (MacDonald and Wilson, 1996; Otteson et al., 1998). Functionally, Pax2 is believed to play a role during optic fissure fusion and the subsequent guidance of ganglion cell axons exiting through the optic disc (Otteson et al., 1998). Given that delayed optic fissure fusion and impeded ganglion cell axon exit are characteristic of the $B s t /+$ phenotype, we looked for indicators of interactions between Pax2 and Bst during the critical developmental period between the time of ganglion cell birth and axon exit. We examined Pax2 expression in the Bst/+ retina, and studied retinal development in a compound heterozygous mutant constructed from $\mathrm{Bst} /+$ and $\mathrm{Krd} /+$ (kidney and retinal defects). $K r d$ is a transgenically induced mutation in which a $7 \mathrm{cM}$ segment of Chromosome 19 containing the Pax2 gene is deleted (Keller et al., 1994). Phenotypically, $\mathrm{Krd} /++$ mice resemble Pax 2 frame-shift mutants and knockouts in terms of the tissues affected and the type of defects observed (Torres et al., 1996; Favor et al.,1996). In the developing $\mathrm{Krd} /+$, optic fissure fusion within the optic stalk is delayed, and there are indications of ganglion cell axon misrouting within the retina (Otteson et al., 1998). We find that Pax2 expression is ectopic in the Bst at E11-12, suggesting that there can be either a loss or a delay in the expression of positional cues in the $B s t /+$ retina. There is 
also significant potentiation of the retinal defects in the compound heterozygous mutants compared to the parental mutants.

We have used the same compound mutant approach to study the effects of Bst in relation to Hes 1, a basic helix-loop-helix (bHLH) molecule that has been shown to play a role in maintaining retinoblasts in the mitotic phase (Tomita et al., 1996). In the Hes $1^{+/-}$ knockout mouse, premature differentiation of retinoblasts occurs by as early as E9.5, leading to numerous defects of the retina that often resemble those seen in the $B s t /+$, such as delayed optic fissure fusion, atrophy of the neural retina within relatively normal-sized eyes, atrophy of the optic nerve, and skeletal malformations affecting the skull and vertebra. Given that there appears to be a delay of cell birth in the $B s t /+$, we hypothesized that Bst and HesI are functional antagonists within the same or parallel molecular pathway(s). Our results hint at such a possibility.

$B s t$ likely plays a critical role within a multi-component, muti-step pathway that regulates the timing of neurogenesis in the retina. The $B s t$ mutation appears to indirectly affect $P a x 2$ expression through delayed cellular differentiation, and partially neutralize the effects of Hesl knockout due to possible functional antagonism between the genes.

\subsection{Materials and methods.}

\subsubsection{Animals and genotyping.}

Hemizygous Belly spot and tail (Bst/+; on C57BLKS/J background) and Kidney and retinal defects $(\mathrm{Krd} /+$; on $\mathrm{C} 57 \mathrm{BL} / 6 \times \mathrm{C} 3 \mathrm{H} / \mathrm{He}$ background) mice were originally obtained from the Jackson Laboratory (Bar Harbor, ME) and mated to their respective wildtypes to initiate breeding colonies. Two hemizygous Hairy and enhancer of split1 knock-out $\left(\mathrm{Hes}^{+/-}\right)$males on the $129 / \mathrm{SV} \times \mathrm{ICR}$ strain background were a gift from Dr. Kageyama (Kyoto, Japan), and were used to seed our Hes 1 breeding colony, from which all experimental animals were derived. All mice were maintained on a 14 hrs: 10 hrs light and dark cycle and fed a standard diet.

$B s t /+$ mice were identified by their kinked tail. $\mathrm{Krd} / \mathrm{+}$ mice were genotyped using genomic DNA obtained from the tail through a "high salt" extraction method. Briefly: A $1 \mathrm{~cm}$ length of the tail was clipped from the mouse and placed in $600 \mu 1$ TNES containing $20 \mathrm{mg} / \mathrm{ml}$ proteinase K (Sigma, St. Louis, MO), then incubated for 8 to 20 hours in a 55

${ }^{\circ} \mathrm{C}$ water bath with gentle agitation. Once the tissue is dissolved, $166.7 \mu \mathrm{l}$ of $6 \mathrm{M} \mathrm{NaCl}$ was added to the solution, and thoroughly mixed through vortexing. The cocktail was allowed 
to stand for 5 seconds and was centrifuged at $14000 \mathrm{rpm}$ for 10 minutes. The supernatant was transferred to a fresh centrifuge tube, into which 1 volume of $95 \%$ ethanol was added. After allowing the DNA to precipitate, it was collected as a pellet via centrifuge. The pellet was then rinsed with $95 \%$ ethanol and allowed to air dry. The DNA pellet was resuspended in TE to a final concentration of 100 to $200 \mathrm{ng} / \mu \mathrm{l}$ as verified through spectrophotometric readings. PCR was used to assay the $K r d$ genotype, using primers targeted to the transgene sequence (Keller et al., 1990). The PCR primers (5'-CTA CCC TGA AAT GTG TGA GAG TTC TGA ACC-3' and 5'-CAT GTG TGA GGT CAA AGC ACA GCT TGT CAG G-3', producing a 500 bp band in $K r d$ mutants) were synthesized at the UT Molecular Resource Center and at St. Jude Children's Research Hospital. The reaction protocol was as follows. A $20 \mu \mathrm{l}$ reaction solution was prepared using 1 to $10 \mathrm{ng}$ of genomic DNA, $1 \mathrm{mM}$ of each primer, $1 \times \mathrm{MgCl}_{2}$-free reaction buffer, $1.5 \mathrm{mM} \mathrm{MgCl} 2,1$ unit of Taq polymerase (Promega, or Perkin Elmer), and $\mathrm{d}_{2} \mathrm{O}$. The reaction mix was gently stirred to ensure even distribution of reagents and DNA, then cycled through the following temperature program using a Perkin Elmer Gene Amp 9600. An initial denaturation step of 3 minutes at $94{ }^{\circ} \mathrm{C}$, then 16 cycles of $\left(30\right.$ seconds at $94{ }^{\circ} \mathrm{C}, 30$ seconds at $50{ }^{\circ} \mathrm{C}, 45$ seconds at $72{ }^{\circ} \mathrm{C}$ ), followed by 20 cycles of ( 30 seconds at $94{ }^{\circ} \mathrm{C}, 30$ seconds at $56^{\circ} \mathrm{C}, 45$ seconds at $72{ }^{\circ} \mathrm{C}$ ), then a final extension step of 6 minutes at $72{ }^{\circ} \mathrm{C}$, followed by cooling to $4{ }^{\circ} \mathrm{C}$. A loading buffer consisting of $60 \%$ sucrose and $5 \mathrm{mM}$ cresyl red was often added the reaction mix prior to PCR, at $13 \mu \mathrm{l}$ per $100 \mu \mathrm{l}$ reaction mix, so as to allow immediate loading of the PCR products to a $0.8 \%$ agarose gel, prestained with ethidium bromide, for electrophoresis. Electrophoresis was carried out at $100 \mathrm{~V}$ for 30 minutes using a mini gel unit (Hoefer Scientific Instruments), DNA was visualized using an ultra violet light box. The size of the DNA fragments were compared against a DNA size marker (Boehringer Mannheim) and the genotype of the animals were determined. Hes + $^{+-}$mice were similarly identified using PCR primers targeted against the recombinant DNA fragment (Ishibasi et al., 1995; 5'-ATG GAT TGC ACG CAG GTT CTG-3' and CTG ATG CTC TTC GTC CAG ATC-3', producing a 476 bp band in Hes 1 mutants).

Retinal anomalies were detected and graded through pupillary-reflex examination, ranging from " 0 " for normal pupillary response (i.e., full contraction of dark-adapted pupil upon bright penlight stimulation) to " 5 " for absence of response. 


\subsubsection{Adult retina collection and analysis.}

At post-natal day $30(\mathrm{P} 30+/-5$ days), animals of known genotype were re-tested for their pupillary reflex, then deeply anesthetized with avertin and perfused transcardially with 4\% paraformaldehyde [PF; in phosphate buffered saline (PBS)]. The heads were removed and post-fixed by immersion in $4 \% \mathrm{PF}$ overnight. The retinas were dissected from the orbit and cut along the ciliary margin to remove the cornea and the lens. The dorsal/ventral and nasal/temporal axes of the retina were determined using the obliquely oriented horizontal arteries. The retinas were flattened after a series of cuts were made in the periphery. They were mounted on slides in gelvitol and examined under a Zeiss microscope. A camera lucida attachment to the microscope was used for tracing the outlines of the retinas, and for making note of the structural anomalies, such as retinal detachment (easily identified as patches of the dissected retina without any attached pigmented epithelium). The traced outline of the retinas was scanned using a digital tablet connected to a Macintosh computer, and the retinal area was computed. Following the gross analysis, the retinas were immediately cut into longitudinal strips near the midline and transferred from the slides to $0.1 \mathrm{M}$ phosphate buffer (PB) in preparation for embedding in Spurr's resin manufacturer (EMS, Ft. Washington, PA). The tissues were embedded following instructions supplied by the manufacturer. The embedded tissues were sectioned at $0.5 \mu \mathrm{m}$, stained with toluidine blue, and examined under a microscope. Images of the sections were captured using a digital camera (Nikon, or Diagnostic Instruments) attached to a microscope and processed through a desktop computer running Photoshop (Adobe). The relative thickness of the retinal layers was measured from the images. Quantification of cell numbers within various retinal layers was performed by averaging the number of cells in equidistant samples taken within those layers. For retinal ganglion cells, the average number of cells per unit distance was compared between groups. For inner nuclear layer (INL) and outer nuclear layer (ONL), the mean number of cells within a single cellular column across the thickness of the respective layer was used for comparison between groups. Statistical comparison of the means was performed using one way analysis of variance (ANOVA), an embedded function of the Excell program (Microsoft) used to compile the database.

5.2.3 Quantification of axon number in the optic nerve.

Optic nerves were obtained from the same adult animals in which retinal analysis was performed. The nerves were embedded in Spurr's resin and sectioned at $75 \mathrm{~nm}$, 
stained with lead, and examined under a transmission electron microscope (JEOL JEM2000 EXII). Twenty three evenly spaced areas were sampled within each optic nerve at $12,000 \times$ magnification and photographed (Fig. 5.1). Myelinated and unmyelinated axons in each micrograph were subsequently counted under a magnifying glass using a square grid, such that axons within the grid and those broaching two of the four boundaries were tabulated. To estimate the total number of axons within the optic nerve, the average axon
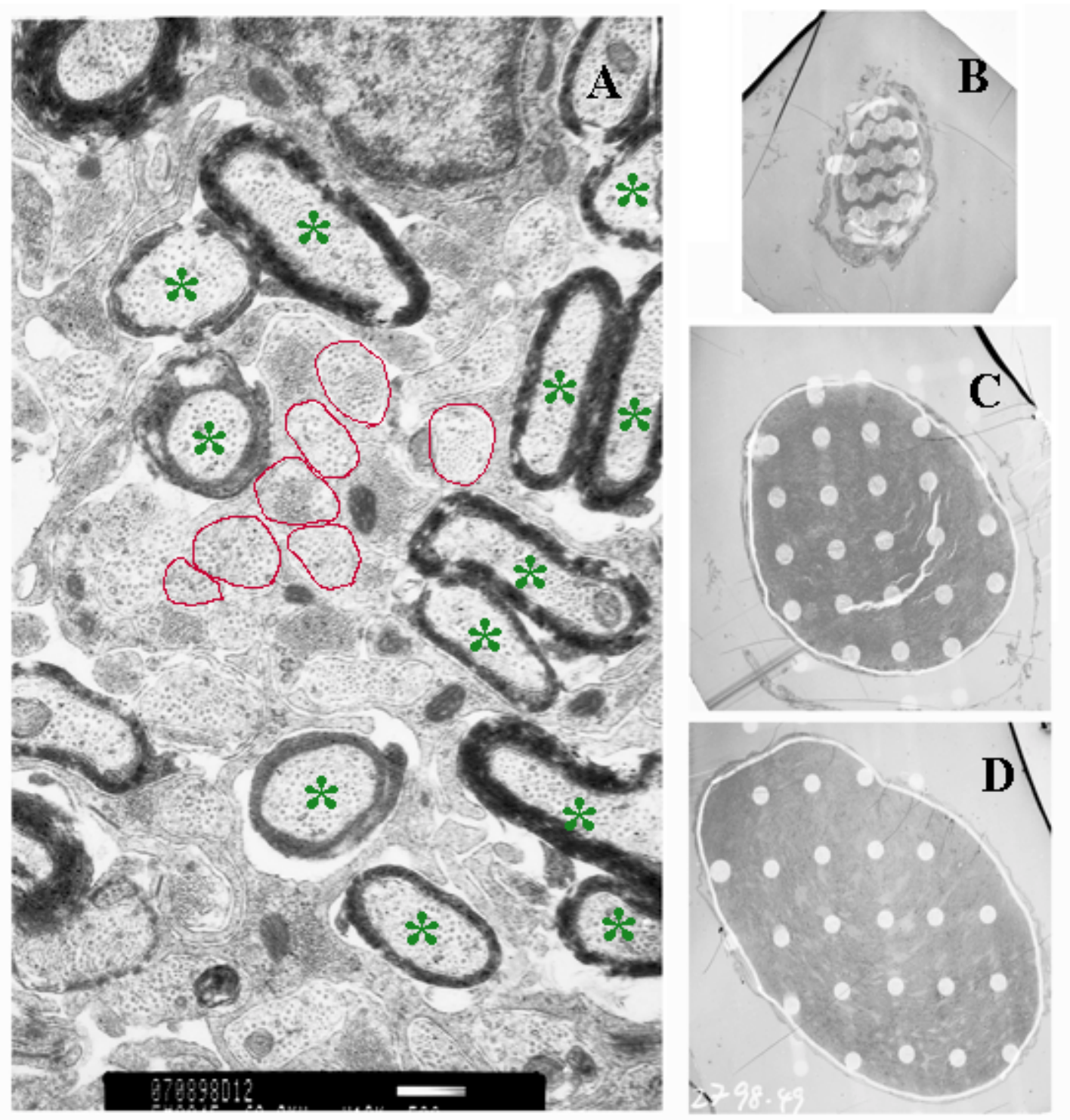

Fig. 5.1 Electronmicrographs of the optic nerve. A) High magnification (12000×) micrograph of a typical optic nerve. B) Low magnification $(250 \times)$ micrograph of optic nerve from a $B s t /+\mathrm{Hes}^{(+/+)}$.C) Second optic nerve from the same individual as B. D) Optic nerve from a Bst/ $++{ }^{K r d} /+$. The myelinated axons in A are marked with green asterices. A cluster of unmyelinated axons are circled in red. Both numbers are used to tabulate the total axon count. The evenly-spaced dots on the lower magnification micrographs are sampled areas. As can be appreciated from the juxtaposition, there are wide differences in the size of optic nerves between the genotypes and occasionally within the same individual. The axon densities in B, C and D are similar. 
density of the 23 sampled areas was multiplied by the area of the nerve section in which the samples were collected. The sectional area was digitally measured from a 200× micrograph using a digital tablet. For each optic nerve and at every magnification, calibration of the measuring areas was performed using micrographs of a 2160 lines $/ \mathrm{mm}$ grid scale (EMS, Washington, PA) and a digital caliper. In order to prevent bias, the analyses of the optic nerve axon number and retinal morphology were conducted without the foreknowledge of the animals' genotype.

\subsubsection{Embryo collection.}

In order to obtain embryos at the desired developmental ages, females in mating cages were checked daily for vaginal plugs. Embryonic day 0 (E0) for a given litter was at 12:00 AM of the day on which the corresponding plug was detected. At E11.5 or E12.5, the timed-pregnant dam was sacrificed by cervical dislocation. The embryos were dissected from the uterus, measured lengthwise, photographed, and immersion fixed in either 1:3 acetic acid/ 95\% ethanol or 4\% PF/PBS, depending on the experimental protocol. Genotyping of the embryos from intercross litters was carried out using DNA extracted from the lower half of the embryos using the same protocols described earlier. $B s t /+$ embryos were identified phenotypically by their shorter and often kinked tail from E12.5 onward, and prior to that, by their smaller stature and external defects, such as failure of neuropore closure.

\subsubsection{RNA collection and Hes1 northern hybridization.}

Total RNA from E12.5 Bst/+ and wildtype whole embryos were extracted using standard methods (Sambrook et al., 1989). The RNA was loaded in $1.5 \%$ formamide gel and electrophoresed at $100 \mathrm{~V}$ for 3 hours, then transferred to a nylon membrane (Hybond) using standard procedures. ${ }^{32} \mathrm{P}$-tagged DNA probes were made using PCR products (HesI exons 1 and 4, obtained using primers designed according to DNA sequences published in Ishibashi et al., 1994) that were random-primer-labeled (Prime-It II, Stratagene). Hybridization was carried out overnight in a rotating chamber at $42^{\circ} \mathrm{C}$ in a hybridization buffer consisting of $0.25 \mathrm{M} \mathrm{Na}_{2} \mathrm{HPO}_{4}$ (pH 7.2), $0.25 \mathrm{M} \mathrm{NaCl}, 7 \%$ SDS, 1mM EDTA, $50 \%$ formamide, $10 \%$ PEG 8000. After the incubation, the blots were washed for 5 minutes at room temperature in $2 \times \mathrm{SSC}$, followed by 2 washes of 20 minutes each at $65^{\circ} \mathrm{C}$ in $0.25 \mathrm{M}$ $\mathrm{Na}_{2} \mathrm{HPO}_{4}(\mathrm{pH} 7.2), 0.2 \%$ SDS, $1 \mathrm{mM}$ EDTA, and 2 rinses of 20 minutes each at $65^{\circ} \mathrm{C}$ in $50 \mathrm{mM} \mathrm{Na}_{2} \mathrm{HPO}_{4}(\mathrm{pH} 7.2), 0.2 \%$ SDS, $1 \mathrm{mM}$ EDTA. The blot were then sealed in plastic 
sleeves and exposed to an X-ray film (Kodak) at $-80{ }^{\circ} \mathrm{C}$ for up to a week depending on the signal strength of the radio-labeled probe.

\subsubsection{Pax2-immunohistochemistry.}

The 4\% PF-fixed E11.5 and E12.5 Bst/+ and control embryos were sucroseprotected overnight and sectioned horizontally at $10 \mu \mathrm{m}$ on a cryostat and mounted on slides (Superfrost/Plus, Fisher Scientific). Other E11.5 and E12.5 embryos were immersion-fixed in 1:3 acetic acid/ethanol and embedded in paraffin, then sagittally sectioned at $6 \mu \mathrm{m}$ and mounted on slides. Polyclonal rabbit anti-Pax2 antibodies were obtained from Zymed (So. San Francisco, CA) and diluted to 1:500 and tested according to supplier recommendations. The reaction protocol was as follows: The slides were flattened and deparaffinized through xylene and ethanol. The hydrated tissues were rinsed 3 minutes in PBS-T ( $3 \mathrm{ml}$ 100\% Triton per $1 \mathrm{~L} \mathrm{PBS}$ ), followed by a 3 minute treatment of $10 \% \mathrm{H}_{2} \mathrm{O}_{2}$ in PBS, then rinsed again in PBS-T. The primary antibody (anti-Pax2) was applied to the slides in a solution consisting of $0.2 \%$ anti-Pax2 stock and 5\% NGS (new goat serum) in PBS-T. Rubber cement was placed around the tissues to maintain the solution above them. The slides were incubated in a humid chamber. After incubation, the slides were rinsed in 3 volumes of PBS-T, after which the secondary antibodies (Vectastain anti-rabbit, Vector Laboratories, Burlingame, CA) were applied. It consisted of a PBS-T solution containing $0.5 \%$ anti-rabbit IgG and 5\% NGS. After 30 minutes of incubation at room temperature, the slides were rinsed in 3 volumes of $0.1 \mathrm{M}$ PB for 10 minutes each, followed by 1 rinse in $0.01 \mathrm{M}$ PB for 10 minutes. The tissues were then reacted with DAB (30 mg diaminobenzidine in $60 \mathrm{ml}$ APB, catalyzed by $800 \mu 1$ of $0.3 \%$ $\mathrm{H}_{2} \mathrm{O}_{2}$ just prior to use) for 10 to 20 minutes. A negative control slide (subjected to identical treatment minus the primary antibody) was included in the run to assure that the positive signals are specific. Once the progress of the detection reaction had reached a satisfactory point, the tissues counter-stained (optional), then dehydrated through ethanol and xylene solutions, and coverslipped using Permount. Photo images of the slides were digitally captured and processed as described earlier. 


\subsection{Results.}

5.3.1 Bst and Pax2 interactions.

5.3.1.1 Pax2 expression is abnormal in the developing $B s t /+$ retina.

As reported elsewhere, $\operatorname{Pax} 2$ expression in the neural retina is largely confined to the ventral area flanking the optic fissure between E11.5 and E12.5 (Torres et al., 1996; Otteson et al., 1998). At the same developmental age, Pax2 expression in the Bst/+ embryos ranges from normal to widely ectopic (Fig. 5.2). In extreme cases, cells of the entire ventral retina of the $B s t /+$ embryo are labeled by the Pax 2 antibody. In less severely symptomatic Bst/+ embryos, the rostral (nasal) portion of the ventral retina appears to have more labeled cells than the caudal (temporal) portion of the retina. In none of the $B s t /+$ embryos examined does the ectopic expression of Pax2 extend to the dorsal retina. Along with the diffuse Pax2 expression pattern is the comparatively thicker wall of the optic stalk in the Bst/+ mouse compared to that of its wildtype littermate (Fig. 5.2). The lumen within the optic stalk is also larger in Bst/+ compared to that of the wildtype.

5.3.1.2 The survival of $B s t /+K r d /+$ compound mutant offspring is compromised.

In $K r d /+\times+{ }^{K r d} /+$ litters, the inheritance of the mutant $K r d$ allele appears to follow the Mendelian pattern during embryonic development (Table 5.1), although fewer $\mathrm{Krdl}+$ embryos than expected appear to actually survive through birth. When $\mathrm{Krd} /+$ mice are mated with $B s t /+$, the number of surviving $\mathrm{Krd} /+$ mice among the $\mathrm{F} 1$ offspring is likewise below the expected, and the ratio between the actual number of surviving mutants and the expected number of mutants is $60 \%$, the same as that seen in the parental $\mathrm{Krd}$ population. 

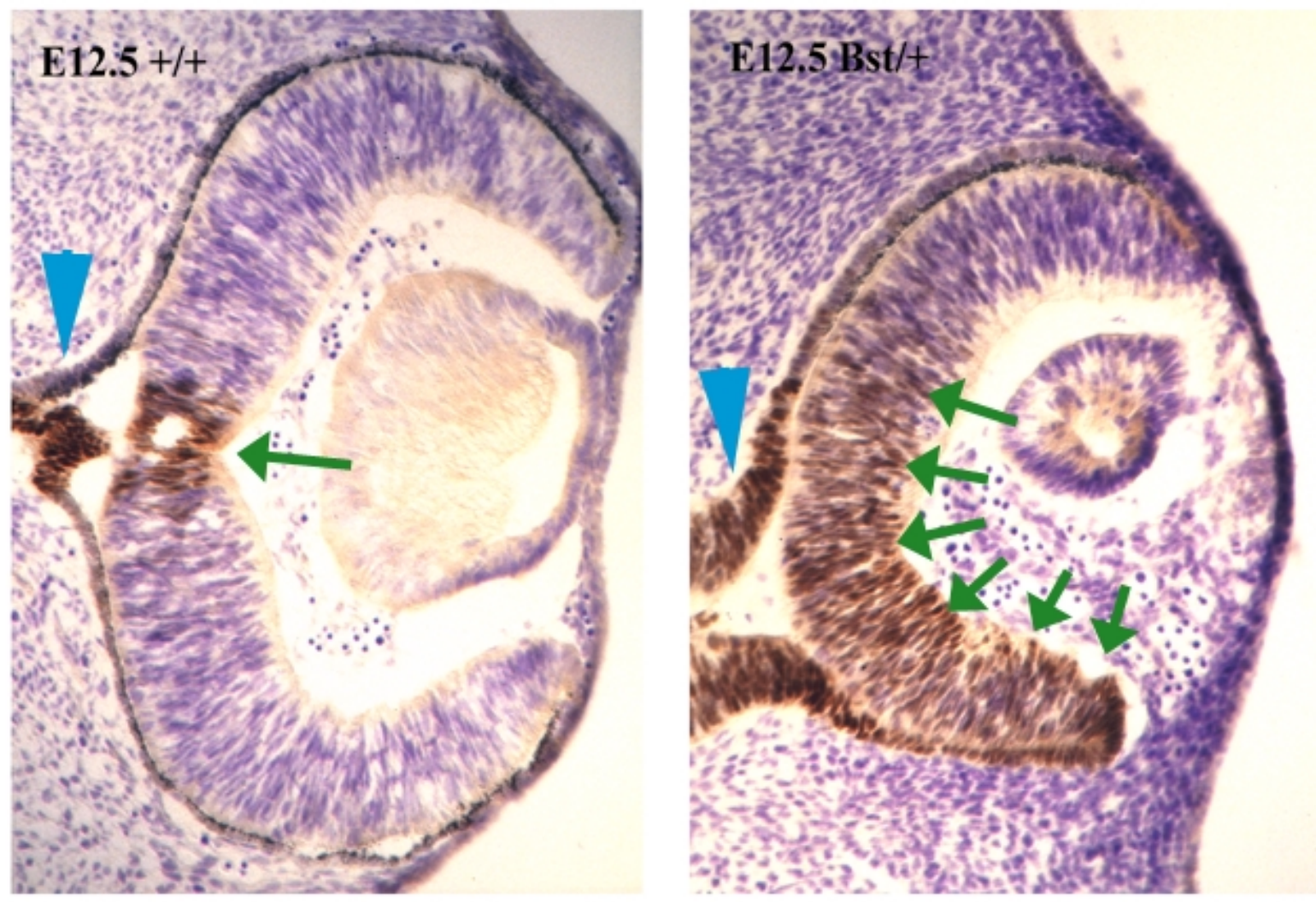

Fig. 5.2 Pax2 expression in the developing Bst/+ retina. Side by side comparison of Pax2 immunolabeling pattern in two horizontally sectioned E12.5 littermate retinas. Green arrows point to tissues expressing Pax2. Quite clearly, Pax2 expression is limited to the optic disc region and the optic stalk in the wildtype, whereas in the Bst/+ retina it is widespread. The ectopic expression of Pax2 is not always prominent in every mutant retina examined, not unlike other variable phenotypes seen in the $B s t /+$. It is also notable that the walls of the optic stalk in the Bst/+ appear considerably thicker than those of the wildtype (light blue arrow heads).

However, all Bst/+ in the same F1 litters appear to survive, which is a significant improvement over the condition in the parental Bst/+ population, where only $72 \%$ of the expected number of $B s t /+$ are accounted for among the born. The genotype-related differences in the survivability of mutant F1 offspring indicates that the Bst-Krd compound strain background is amenable to the survival of $B s t /+$ mutants but not to that of $\mathrm{Krd} / \mathrm{+}$ mutants. Given this observation, it is noteworthy that only $36 \%$ of the expected number of $B s t /+K r d /+$ compound heterozygous mutants are seen in the litters, suggesting that the combined effect of $B s t$ and $K r d$ mutant alleles is more deleterious to the animal's survival than either of the individual mutant alleles alone, given similar genetic background. The compound heterozygous mutants are also visibly smaller than their littermates (Fig. 5.3). 
Table 5.1. Compound mutants litter composition.

\begin{tabular}{|c|c|c|c|c|c|c|c|c|c|c|c|}
\hline $\begin{array}{c}\text { parent } \\
1\end{array}$ & parent 2 & age & $\begin{array}{l}\text { litter } \\
\text { number }\end{array}$ & $\begin{array}{l}\text { total } \\
\text { animals }\end{array}$ & $\begin{array}{l}\text { Bst/+ } \\
\text { survival }^{(1)}\end{array}$ & $\begin{array}{l}\mathrm{Krd} /+ \\
\text { survival }^{(1)}\end{array}$ & $\begin{array}{l}\text { Bst/Krd } \\
\text { survival }^{(1)}\end{array}$ & $\begin{array}{l}\text { Hes1/+ } \\
\text { survival }^{(1)}\end{array}$ & $\begin{array}{l}\text { Bst/Hes1 } \\
\text { survival }^{(1)}\end{array}$ & polydactyly ${ }^{(2)}$ & exencephaly ${ }^{(2)}$ \\
\hline \multirow[t]{2}{*}{ Bst/t } & $+/+$ & E11-E13 & 9 & 70 & $79 \%$ & - & - & - & - & - & $14 \%$ \\
\hline & & P0-adult & 36 & 236 & $70 \%$ & - & - & - & - & $36 \%$ & - \\
\hline \multirow[t]{2}{*}{$\mathrm{Krd} /+$} & $+/+$ & E12 & 2 & 14 & - & $100 \%$ & - & - & - & - & - \\
\hline & & P0-adult & 7 & 37 & - & $61 \%$ & - & - & - & - & - \\
\hline Bst $/+$ & $\mathrm{Krd} /+$ & all & 28 & 126 & $100 \%$ & $60 \%$ & $26 \%$ & & - & $5 \%$ & $3 \%$ \\
\hline $\operatorname{Hes} 1^{+/-}$ & $+/+$ & P0-adult & 8 & 53 & - & - & - & $50 \%$ & - & - & - \\
\hline \multirow[t]{2}{*}{$\operatorname{Hes}^{+/ *}$} & Hes $1^{+/ .}$ & E13 & 4 & 42 & - & - & - & $83 \%{ }^{(3)}$ & - & - & - \\
\hline & & P0-adult & 10 & 63 & - & - & - & $16 \%$ & - & - & 一 \\
\hline \multirow[t]{2}{*}{ Bst/+ } & $\mathrm{Hesl}^{+/-}$ & E13 & 3 & 25 & $70 \% \%^{(4)}$ & - & 一 & $89 \%{ }^{(5)}$ & - & - & - \\
\hline & & P0-adult & 39 & 159 & $100 \%$ & - & - & $85 \%$ & $43 \%$ & $20 \%$ & $9 \%$ \\
\hline
\end{tabular}

${ }^{(1)}$ Survival ratio is the proportion between the actual number of animals and the expected number of animals at the time of count. This is based on the assumption that the survival ratio of the wildtype is at or close to $100 \%$. Therefore,

in a litter of 10 from a mutant $x$ wildtype mating containing 3 mutants and 7 wildtype offspring, it is assumed that should all the mutants have survived $(100 \%)$, there would have been 7 mutants and 7 wildtypes; the viability of the mutant offspring is $3 / 7$, or $43 \%$. In the case of Bst/ offpsring, they are actually more numerous than the wildtype littermates in $\mathrm{Bst} /+\mathrm{x} \mathrm{Krd} /+$ and $\mathrm{Bst} /+\mathrm{x} \mathrm{Hes}^{+/-}$litters, resulting in $100 \%$ viability ratio.

${ }^{(2)}$ Polydactyly and exencephaly are only observed in the Bst/+. The \% ratio are based on the number of Bst + in each genotype group.

${ }^{\text {(3) }}$ Both $\mathrm{H}^{\mathrm{esl}+/-}$ and $\mathrm{Hesl}^{-f-}$ are represented in this group.

${ }^{(4)}$ Both Bst $/+/ \mathrm{Hesl}^{+/-}$and Bst/+/Hes1 ${ }^{+/+}$are represented in this group.

${ }^{(5)} \mathrm{Both} \mathrm{Bst} /+/ \mathrm{Hes} 1^{+/-}$and $+/+^{\mathrm{Bst}} / \mathrm{Hes} 1^{+/-}$are represented in this group. 

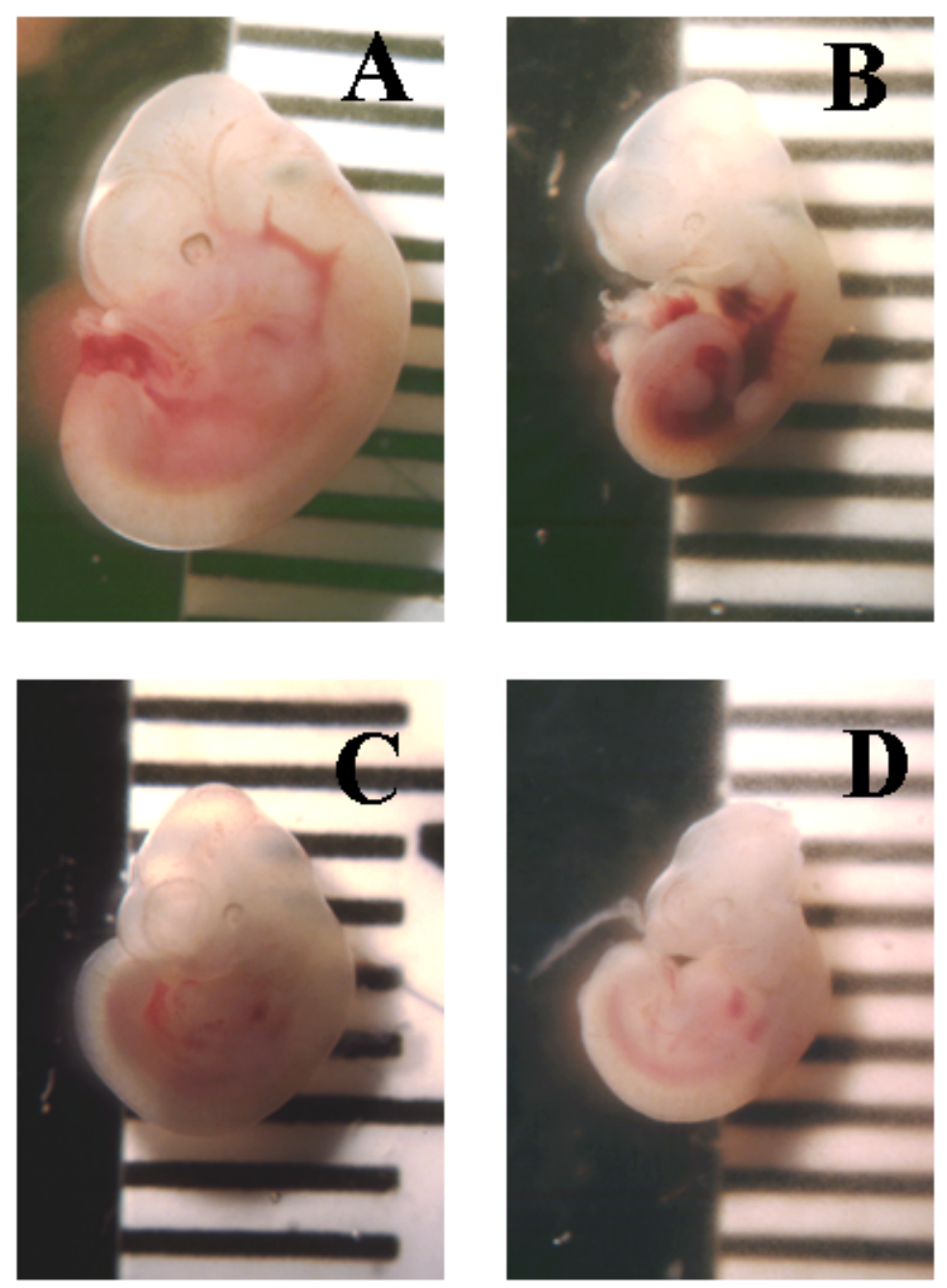

Fig. 5.3 The developing $B s t /+K r d /+$ compound mutant embryos. All four panels show E12.5 littermates from Bst/ $+\times$ $\mathrm{Krd} /+$ mating. A and $\mathrm{B}$ are $\mathrm{Krd} /+$. C and D are $B s t /+K r d /+$ compound mutants. Wildtype embryos are the same size as $\mathrm{A}$, as are $\mathrm{Bst} /+$ $+{ }^{K r d} /+$. It is readily apparent that while a number of $\mathrm{Krd} /+$ mutants are developmentally normal , none of the compound mutants are. Even though the phenotypes vary, all compound mutants eventually develop retinal defects. In contrast, the majority of $B s t /++{ }^{K r d} /+$ have relatively normal eyes. Background ruler is graduated in $\mathrm{mm}$.

Aside from compromised survival ability, the mutant F1 mice also exhibit birth defects similar to those seen in the parental mutants. However in the case of the $B s t /+$, the incidence of such defects is noticeably lower among the F1 mutants than among the parental counterparts. For instance, only $5 \%$ of $B s t /++{ }^{\mathrm{Krd}} /+$ exhibit polydactyly, compared to $36 \%$ of the parental $B s t /+$. Also, only $3 \%$ of the $B s t /++{ }^{\mathrm{Krd}} /+$ are born with exencephaly, which is considerably less than the $13 \%$ observed in the parental $B s t$ litters.

5.3.1.3 The $B s t /+K r d /+$ compound mutation results in increased incidence of eye defects.

The retinal phenotype of $\mathrm{Krd} /+\times \mathrm{Bst} / \mathrm{\textrm {F } 1}$ offspring is noticeably segregated according to their genotype (Fig. 5.4). Among $K r d /++{ }^{B s t} /+$ F1 mice, $27 \%$ show partially 
anomalous reflex in at least 1 eye, while in another $65 \%$ there is complete lack of pupillary reflex and optic nerves. This represents a somewhat increased incidence and severity of eye defects compared to what we have observed in the parental population, although the overall ratio of animals exhibiting any form of eye defects remains essentially unchanged (Table 5.2). In the $B s t /++{ }^{K r d} /+$, there is a $75 \%$ reduction in the incidence of partial pupillary reflex defects compared to the parental $B s t /+$, and the incidence of complete bilateral absence of optic nerves is reduced from $40 \%$ to zero. In stark contrast, all $12 \mathrm{Bst} /+\mathrm{Krd} /+$ compound mutants exhibit bilateral absence of pupillary reflex, and all but one have no optic nerves. In the lone exception, only a vestigial unilateral nerve was observed.
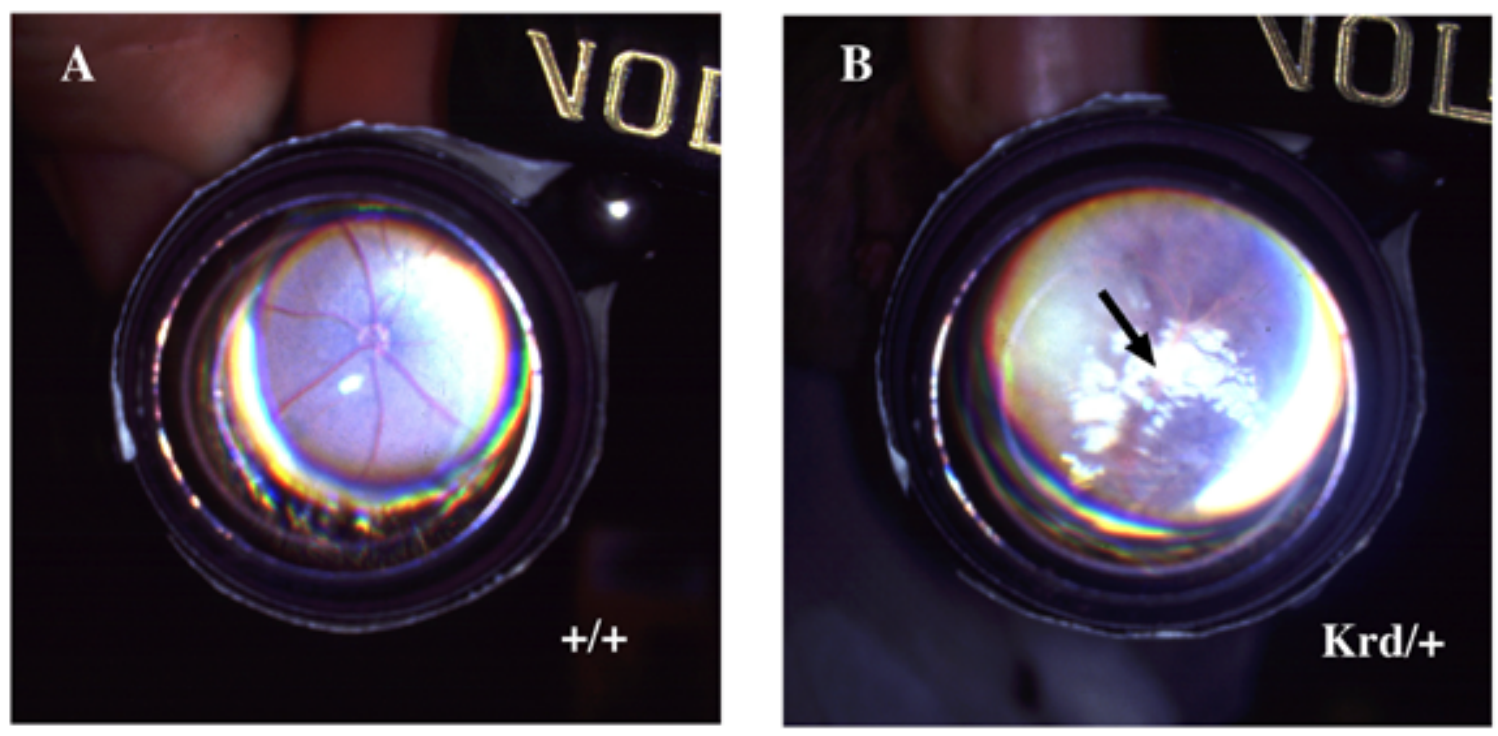

Fig. 5.4 Fundus camera view of the $K r d /+$ retina. A offers a view of a wildtype fundus. B shows that of a $\mathrm{Krd} / \mathrm{+}$. Compare the $\mathrm{Krd} / \mathrm{+}$ retina to that of the Bst/+ in Fig. 3.3, and notice the surface irregularities near the optic disc (arrow). There are also precipitated particulates in the vitreous of the $\mathrm{Krd} /$ + eye that are indicative of retinal detegeneration.

Fundus camera examination of $\mathrm{Krd} /++$ and $B s t /+\mathrm{Krd} /+$ mice exhibiting aberrant pupillary response revealed ring-shaped anomalies that likely represent folds within the neural retina (Fig. 5.4), as well as opaque precipitants within the vitreous, characteristic of retinal degeneration. In all mutants lacking pupillary reflex, there are seemingly apigmented patches in the fundus that likely indicate retinal detachment from the pigmented epithelium. 
Table 5.2. Incidence of pupillary reflex defects in the mutant mice

\begin{tabular}{|c|c|c|c|c|c|c|c|c|c|c|c|}
\hline parent 1 & parent 2 & $\begin{array}{l}\text { \%Bst with } \\
\text { partial pup. } \\
\text { reflex }^{(1)}\end{array}$ & $\begin{array}{l}\text { \%Bst with } \\
\text { no pup. } \\
\text { reflex }\end{array}$ & $\begin{array}{l}\% \text { Krd with } \\
\text { partial pup. } \\
\text { reflex }\end{array}$ & $\begin{array}{l}\text { \%Krd with } \\
\text { no pup. } \\
\text { reflex }\end{array}$ & $\begin{array}{l}\% \text { Bst/Krd } \\
\text { with partial } \\
\text { pup. reflex }\end{array}$ & $\begin{array}{l}\text { \%Bst/Krd } \\
\text { with no } \\
\text { pup. reflex }\end{array}$ & $\begin{array}{l}\text { \%Hes } 1 \\
\text { with partial } \\
\text { pup. reflex }\end{array}$ & $\begin{array}{l}\text { \%Hesl } \\
\text { with no } \\
\text { pup. reflex }\end{array}$ & $\begin{array}{l}\% \text { Bst/Hesl } \\
\text { with partial } \\
\text { pup. reflex }\end{array}$ & $\begin{array}{l}\% \text { Bst/Hesl } \\
\text { with no } \\
\text { pup. reflex }\end{array}$ \\
\hline Bst/t+ & $+/ t$ & $30 \%(89)$ & $40 \%(89)$ & - & - & - & - & - & - & - & - \\
\hline $\mathrm{Krd} /+$ & $+/+$ & - & - & $55 \%(37)$ & $40 \%(37)$ & - & - & - & - & - & - \\
\hline Bst/+ & $\mathrm{Krd} /+$ & $7 \%(30)$ & $0 \%(30)$ & $27 \%(26)$ & $65 \%(26)$ & $0 \%(12)$ & $100 \%(12)$ & - & - & - & - \\
\hline $\mathrm{Hes}^{+/-}$ & $+/+$ & - & - & - & - & 一 & - & $8 \%(25)$ & $4 \%(25)$ & - & - \\
\hline Bst/+ & $\mathrm{Hes}^{+/-}$ & $25 \%(27)$ & $13 \%(27)$ & - & - & - & - & $0 \%(27)$ & $0 \%(27)$ & $21 \%(19)$ & $0 \%(19)$ \\
\hline
\end{tabular}

${ }^{(1)}$ Normal pupillary reflex is defined as complete contraction of a fully dillated pupil upon penlight stimulation. On a scale of 0 to 5 , with 0 being full contraction and 5 being no contraction, normal animals would be phenotyped as $0 / 0$. Animals with bilateral absence of pupillary reflex are $5 / 5$. All other animals, from $0 / 5$ to $1 / 1$, are considered to have partial pupillary reflexes. These animals typically have asymmetric retinal defects and optic nerve hypotrophy. The number of mutants in each given genotype is listed in the parentheses, 
5.3.1.4 The $B s t /+K r d /+$ compound mutation exacerbates retinal and optic nerve atrophy.

Similar to what has been observed in parental mutant animals, there is a clear correlation between the degree of pupillary reflex defect and the extent of retinal and optic nerve atrophy in the F1 mice. The lack of pupillary reflex typically corresponds to severe dysmorphism of the retina and an absence of the optic nerve; whereas attenuated pupillary responses are associated with less severe retinal and optic nerve defects.

In terms of retinal area, the differences between the genotypes are relatively small but consistent (Figs. 5.5, 5.6). The area measured ranges from a low extreme of 11.37 $\mathrm{mm}^{2}$ to a high of $17.94 \mathrm{~mm}^{2}$, with the wildtype and $B s t /++{ }^{K r d} /+$ mice having the largest retinas, averaging $16.5 \mathrm{~mm}^{2}$. The $K r d /++{ }^{B s t} /+$ retinas are somewhat smaller at just over $16.1 \mathrm{~mm}^{2} . B s t /+K r d /+$ compound mutant retinas are visibly and significantly smaller than those of the other groups, averaging only $15.1 \mathrm{~mm}^{2}$ (ANOVA, $\mathrm{p}<0.005$ ). None of these animals are microphthalmic.

Within the retina, differences between groups are most clearly seen in terms of retinal ganglion cell density, inner plexiform layer (IPL) thickness, and inner nuclear layer (INL) cell density (Fig. 5.6). The widths of outer plexiform layer (OPL), outer nuclear layer (ONL), and the photoreceptor inner- and outer-segment layers are not statistically different between the genotypes. In general, wildtype retinas consistently have the highest density of retinal ganglion cells, the thickest IPL, and the greatest number of cells in the INL. The measurements obtained in the wildtype are closely approximated by those from the $B s t /++{ }^{K r d} /+$ retinas, which are in turn trailed by those of the $K r d /++{ }^{B s t} /+$ retinas. The compound mutant retinas have the lowest scores in all measured areas. In terms of ganglion cell number, IPL thickness, and INL cell number, the differences between the compound mutants and other groups are highly significant (ANOVA, $\mathrm{p}<0.00001$ ). For instance, the thickness of the IPL in the compound mutants is only $30 \%$ to $50 \%$ of that of other genotypes (Fig. 5.6). As mentioned above, the degree of pupillary reflex defect mirrored the level of anatomical anomaly, such that animals with less pupillary response also have thinner retina and fewer cells in the ganglion and INL layers.

One notable feature of $B s t /++{ }^{K r d} /+, K r d /++{ }^{B s t} /+$, as well as $B s t /+K r d /+$ double mutant retinas is the presence of short stretches of greatly thickened regions. These may correspond to the "rings" observed through the fundus camera. Within these short stretches of the retina of typically no more than $100 \mu \mathrm{m}$ in width, the retina may be more than 50\% thicker than adjacent regions. The differences appear to arise almost entirely from a thickened and more cellularized ONL, which is normally 11 to 13 cells thick, 

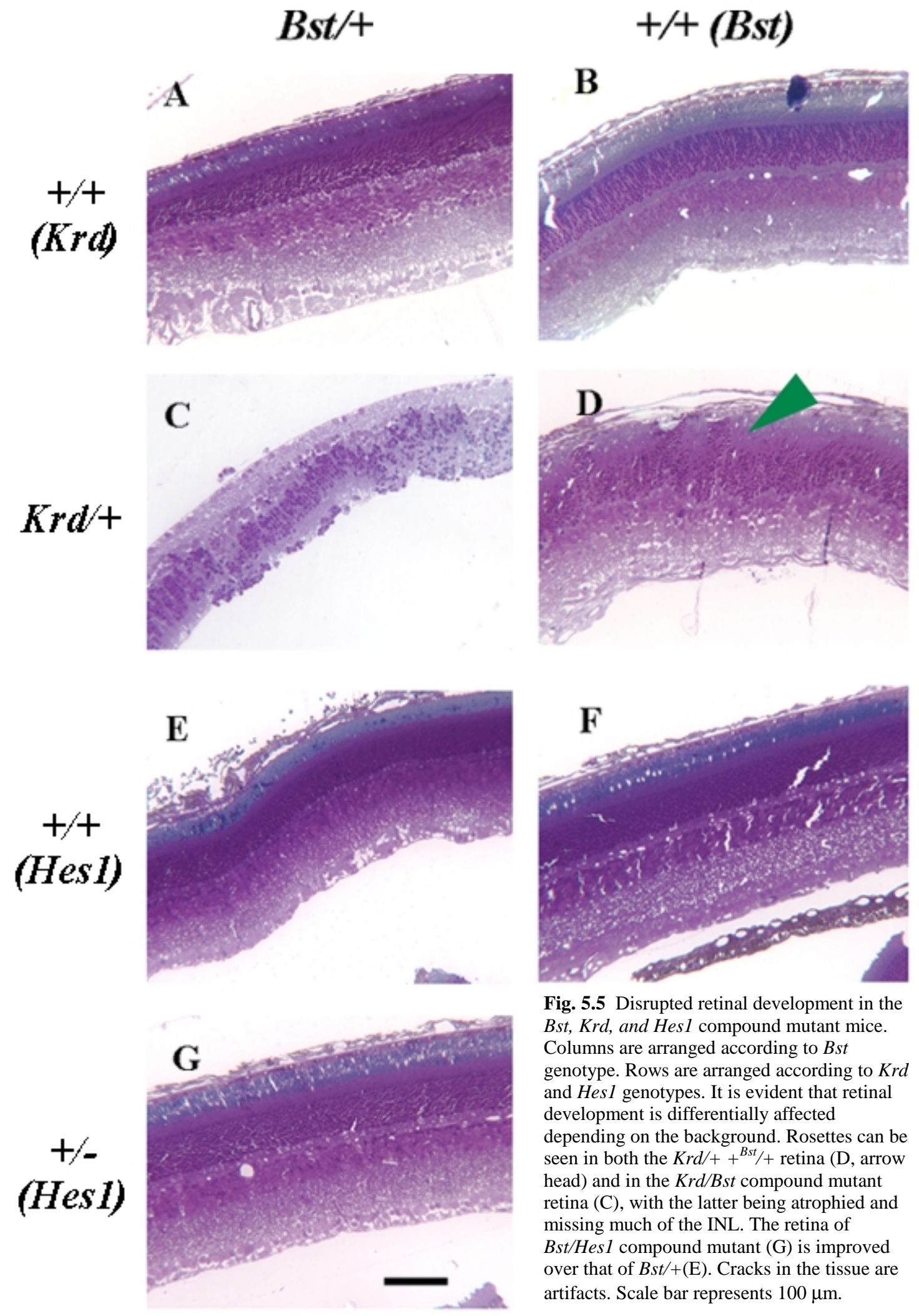

Fig. 5.5 Disrupted retinal development in the Bst, Krd, and Hesl compound mutant mice. Columns are arranged according to Bst genotype. Rows are arranged according to $\mathrm{Krd}$ and Hes 1 genotypes. It is evident that retinal development is differentially affected depending on the background. Rosettes can be seen in both the $K r d /++{ }^{B s t} /+$ retina (D, arrow head) and in the $K r d / B s t$ compound mutant retina $(\mathrm{C})$, with the latter being atrophied and missing much of the INL. The retina of Bst/Hes 1 compound mutant $(\mathrm{G})$ is improved over that of $B s t /+(\mathrm{E})$. Cracks in the tissue are artifacts. Scale bar represents $100 \mu \mathrm{m}$. 
Fig. 5.6 The retinal and optic nerve characteristics of Bst and $\mathrm{Krd}$ mutant mice. Abbreviations: $\mathrm{B} / \mathrm{K}(B s t /+K r d /+) ; \mathrm{B} /+\left(B s t /++{ }^{K r d} /+\right) ;+/ \mathrm{K}\left(+{ }^{B s t} /+K r d /+\right) ;+/+$ (wildtdype); RGC (retinal ganglion cells); IPL (inner plexiform layer); INL (inner nuclear layer); OPL (outer plexiform layer); ONL (outer nclear layer); OS (photoreceptor outer segment layer). Numbers above error bars indicate the number of animals sampled. Cell counts in INL and ONL refer to the mean number of cells in cell columns spanning the respective layers. RGC and INL are the most affected cell layers in the mutant retina. B/+ are noticeably healthier than $\mathrm{K} /+$, while $\mathrm{B} / \mathrm{K}$ exhibits considerable atrophy compared to other groups. 
Bst $\times$ Krd $F 1$ retinal area

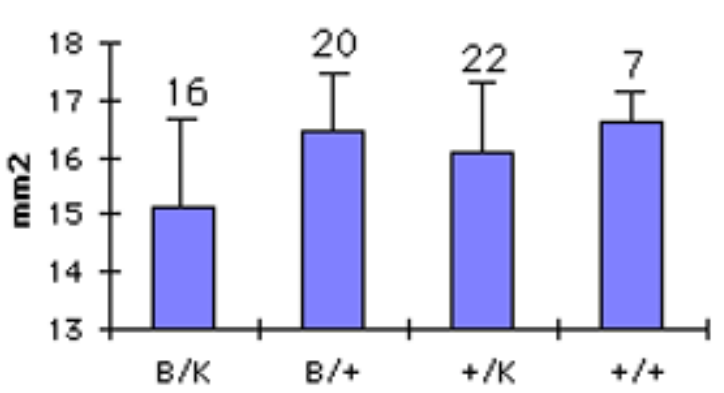

RGC density

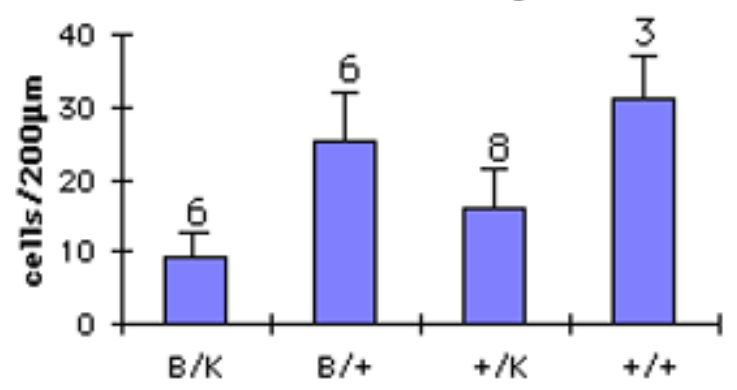

INL cell number

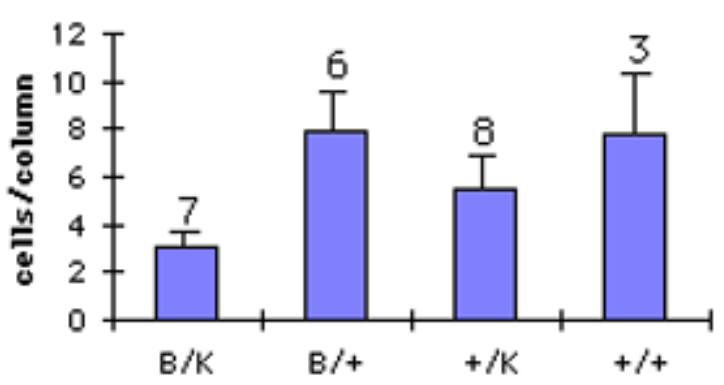

ONL cell number

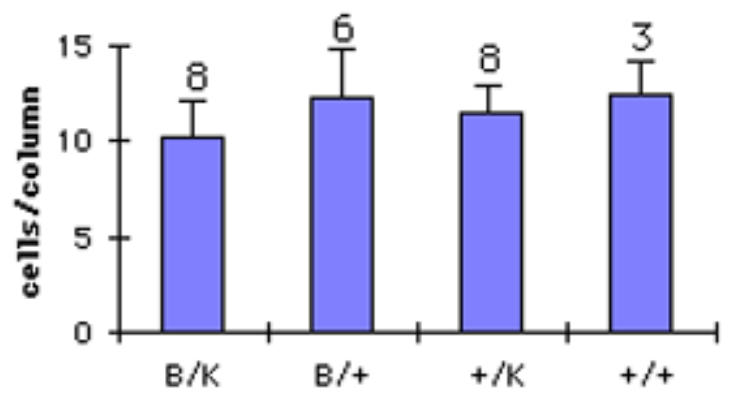

optic nerve axon number

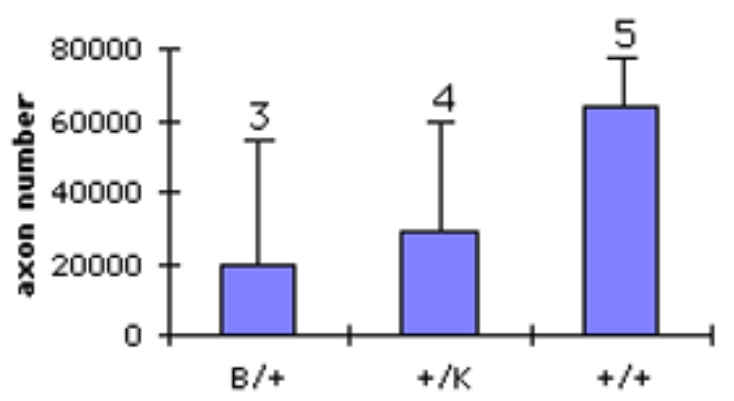

IPL thickness

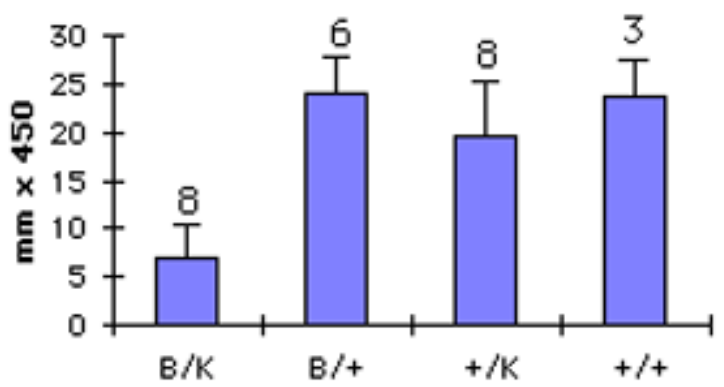

OPL thickness

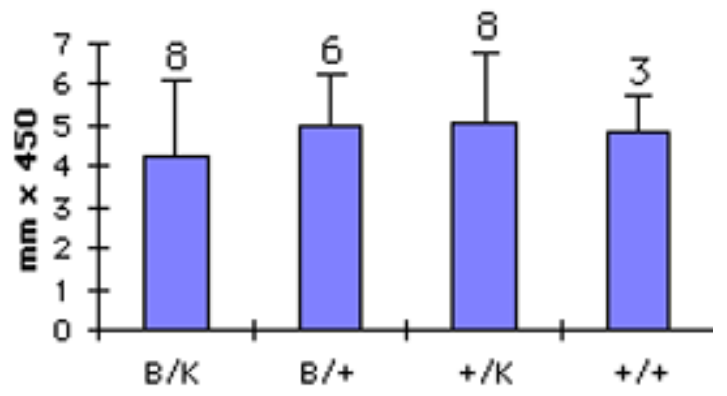

OS thickness

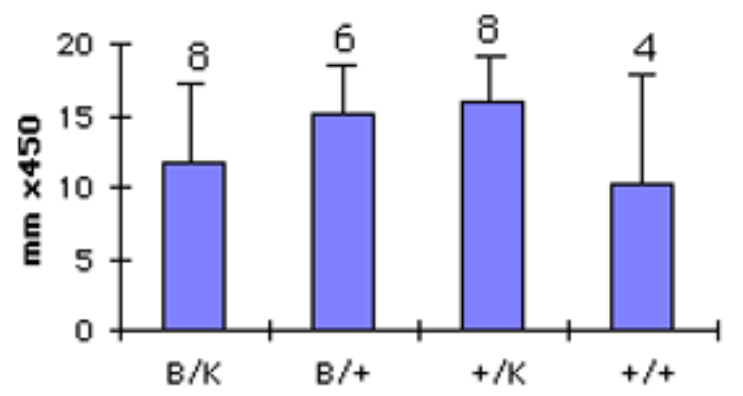


regardless of the genotype, but may be as many as 33 cells thick within the aberrant regions. This is not likely explained by variations in the plane of section caused by folds in the retina, given that the numbers of INL and retinal ganglion cells in the thickened regions do not differ noticeably from those of adjacent areas.

As expected, the number of axons within the optic nerve also exhibit clear correlation with the integrity of the pupillary reflex (Fig. 5.6). To a large degree, we have found that the density of axons within the optic nerves is not affected by the nerve size. As a result, the cross-sectional area of the nerve is a relatively reliable predictor of the number of axons it contains. In the wildtype, the optic nerves are of uniform size and the number of axons per nerve is consistently between the 60,000 to 70,000 . In the $B s t /++{ }^{K r d} /+$, the appearance of the optic nerve is typically indistinguishable from that of the wildtype. Among the $30 \mathrm{Bst} / \mathrm{t}$ $+{ }^{K r d} /+\mathrm{F} 1$, only a single animal has a unilaterally hypotrophied optic nerve, while a second animal has partially degraded pupillary reflex without immediately identifiable optic nerve defects (Table 5.2). The number of axons in the $B s t /++{ }^{K r d} /+$ optic nerve also closely approximates that of the wildtype. In the $26 \mathrm{Krd} /++{ }^{B s t} /+\mathrm{F} 1$ however, 17 mice completely lack pupillary reflex and have no optic nerves. Of the remaining 9 mutants, two have normal reflex and normal optic nerves, the others have partial pupillary reflexes affecting one or both eyes and have nerves of varying sizes, each containing between 7,000 to 70,000 axons. Only 1 of the $12 \mathrm{Bst} /+\mathrm{Krd} /+$ compound mutant mice has a vestigial optic nerve which contains a negligible number of axons. However, given that even in the absence of optic nerves some retinal ganglion cells are present in the corresponding $B s t /+K r d /+$ retinas well beyond P30, it is likely that the axons from these cells are misrouted or trapped within the eye.

\subsubsection{The interactive effects of Bst and Hes1 mutations.}

\subsubsection{Hes 1 expression is likely normal in the $B s t /+$.}

The level of Hes 1 mRNA expression is comparable between E12.5 Bst/+ and wildtype embryos (Fig. 5.7). A $5 \mathrm{~kb}$ band is detected on RNA northern blots prepared from whole embryos of both genotypes, using probes made from PCR products and from Hes 1 cDNA (courtesy of Dr. Kageyama). The fact that both the size of the band and the relative intensity of the label are similar between the mutant and the wildtype suggest that Hes I mRNA is normally synthesized in the $B s t /+$. However, these results do not discount the possibility that there may be small differences in the Hesl sequence between the mutant and the wildtype, such as point mutation or small deletion, or that Hesl mRNA could be 
post-transcriptionally affected by Bst. It is also possible that there may be differences in Hes 1 expression at an earlier developmental stage.

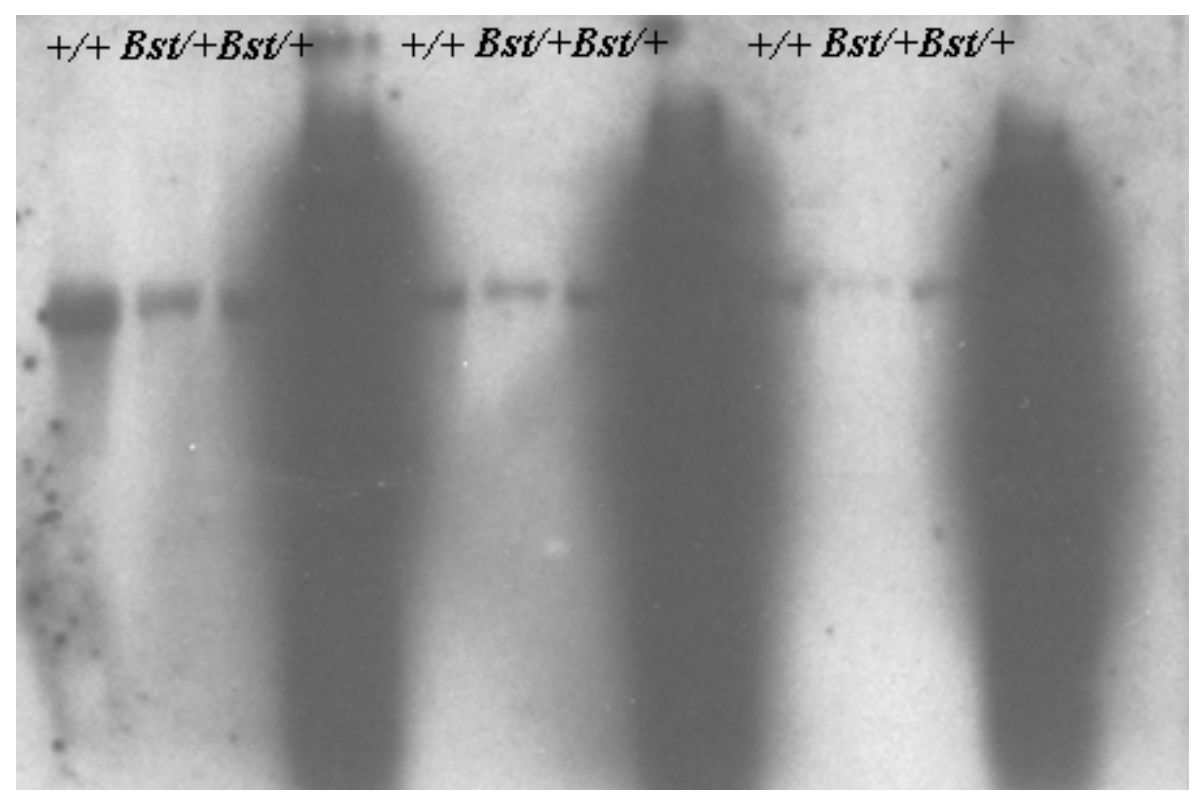

Fig. 5.7 Hesl RNA expression in the Bst/+ mutant mouse. Radiolabeled Hes 1 probe was used to screen a northern blot prepared from total RNA extracted from E12.5 littermate embryos. The three sets of lanes represent a dillution gradient. A single $5 \mathrm{~kb}$ band is seen in all animals.

5.3.2.2 The combined effect of Hesl and Bst mutations on survival is complex.

In contrast to what has been observed in $B s t /+\times K r d /+F 1$ offspring, where mutant animals tend to be smaller than wildtype littermates, the F1 and N2 $(\mathrm{F} 1 \times$ parental backcrosses) newborns from $B s t /+\times \mathrm{Hes}^{+/-}$crosses are uniformly large, and those who survive the immediate post-natal period are healthy. Nevertheless, as with $B s t / K r d \mathrm{~F} 1$ offspring, all birth defects seen in the parental Bst are also present in the F1 and N2 newborns, such as polydactyly and exencephaly, albeit occurring at a reduced frequency (Table 5.2). The number of identifiable Bst/+ among E13.5 F1 embryos is close to the $50 \%$ expectation, and the post-natal survival of $B s t /+\mathrm{F} 1$ is effectively $100 \%$ (Table 5.1). This indicates that the survival capacity of $B s t /+$ is improved by the mixed strain background. The number of Hesl ${ }^{+/} \mathrm{F} 1$ and N2 in E13 and later litters is between $85 \%$ to $90 \%$ of the expected, which is slightly higher than the percentage of $\mathrm{Hesl}^{+/}$among $\mathrm{Hesl}^{+/}$in the breeding litters (Table 5.1), although there is little difference between the breeding litter parents and the F1 litter parents in terms of their respective strain background.

The average embryonic litter size is just over 8 at E13.5; however, there are often embryos which exhibit severe developmental defects that are not likely to survive until 
birth (Fig. 5.8). Among the embryos which fail to survive are likely a number of $B s t /+$ Hesl ${ }^{+/-}$compound mutants, among which only $43 \%$ of the expected are accounted for in the post-natal population. Indeed, about $80 \%$ of the very small embryos (compared to the littermate size) are genotypically $\mathrm{Hes}^{+/}{ }^{+-}$, making it quite possible that a substantial number of them are double mutants.

5.3.2.3 Bst/ $+\mathrm{Hesl}^{+/-}$compound mutation results in mild ocular defects.

While it appears that the compound mutant background improves the survivability of Bst/+ and HesI ${ }^{+/}$but not necessarily that of the compound mutants, the effect of the compound mutation on the eye phenotype is perhaps even more complex. The mice in our Hes 1 breeding colony show inherent retinal defects independent of the mutant Hes 1 allele, of the 45 adult mice genotyped as wildtype $\left(\mathrm{Hesl}^{+/+}\right)$, eight (18\%) exhibit partial to complete lack of pupillary reflex, with corresponding degrees of optic nerve atrophy. However, in the F1 and N2 litters, all surviving wildtype mice have normal pupillary reflex and optic nerves (Table 5.2). Likewise, while about $12 \%$ of the Hesl ${ }^{+/}$in the breeding litters exhibit pupillary reflex anomaly, none of the $+{ }^{B s t} /+\mathrm{Hesl}^{+/}$exhibits such anomaly in the F1 and N2 litters, even though the parental and offspring strain backgrounds are equally mixed. The improvement in pupillary reflex phenotype is less remarkable in the Bst/+ Hesl ${ }^{+/+}$but still quite apparent over that of the parental Bst/+ (Table 5.2). The surviving compound mutants also exhibit eye defect, but interestingly with a lower incidence and to a lesser degree than in the $B s t /+\mathrm{Hesl}^{+/+}$.

5.3.2.4 Bst/+ Hes $1+/-$ compound mutants have relatively healthy retinas and optic nerves.

Retinal surface area ranges from $13.1 \mathrm{~mm}^{2}$ to $17.7 \mathrm{~mm}^{2}$ among the $\mathrm{F} 1$ mice. As a group, $+{ }^{\mathrm{Bst}} /+\mathrm{Hesl} \mathrm{I}^{+/}$have the largest retinas, averaging nearly one full square millimeter larger than the wild type retinas (Fig. 5.9). The wildtype retinas are on average larger than those of the compound mutants, which are in turn larger than those of the Bst/+ Hesl ${ }^{+/+}$. There is much overlap between the groups with the exception of the $B s t /+$, which is significantly different from Hes1+/- (ANOVA, p=0.02).

At the ultrastructural level, the order of difference between groups remains the same (Fig. 5.9). The measurements of the thickness of various retinal layers and the number of cells within these layers are quite close and there is much overlap between groups, with $+{ }^{\mathrm{Bst}} /+\mathrm{HeSI}^{+/}$and wildtype data being essentially interchangeable, and $\mathrm{Bst} /+$ 

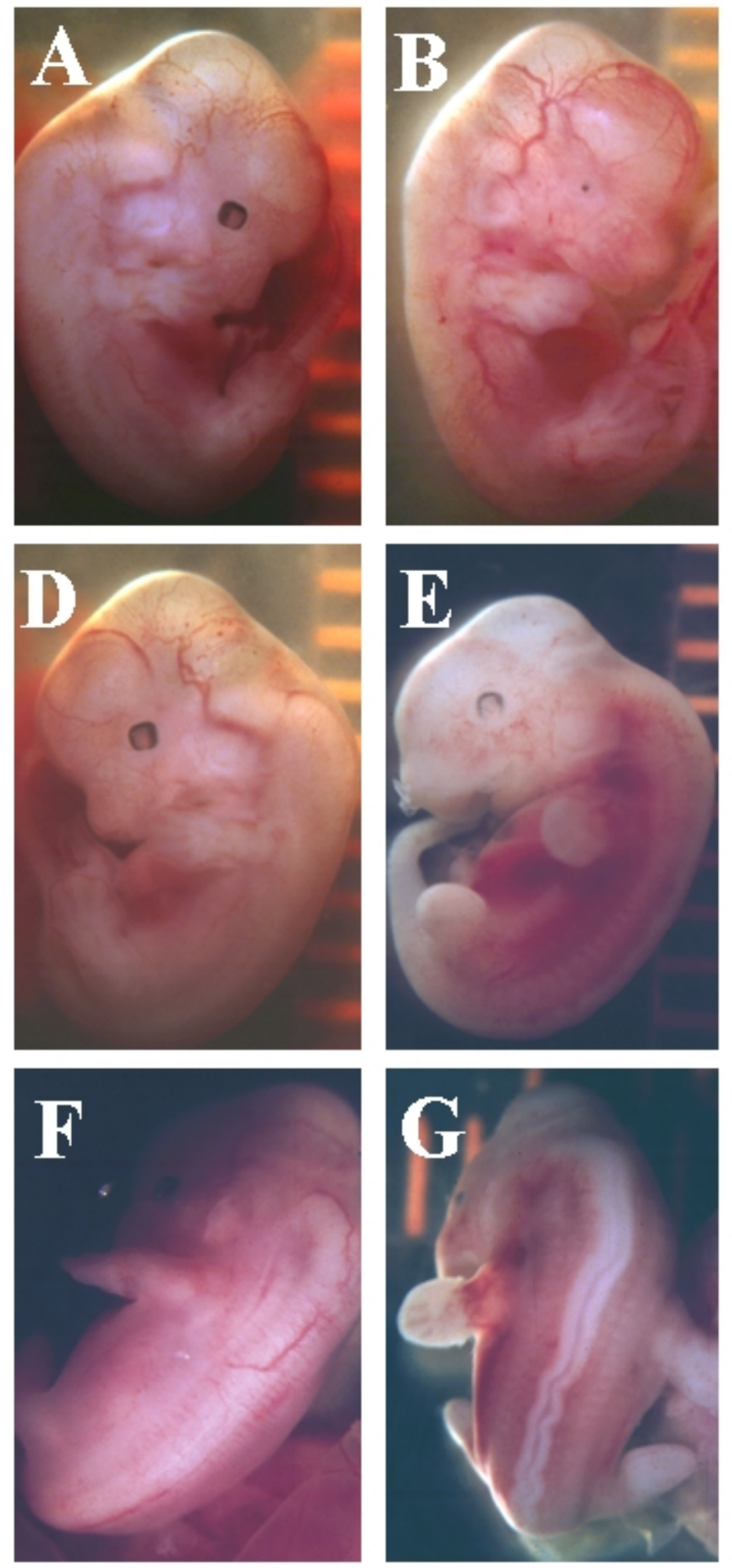

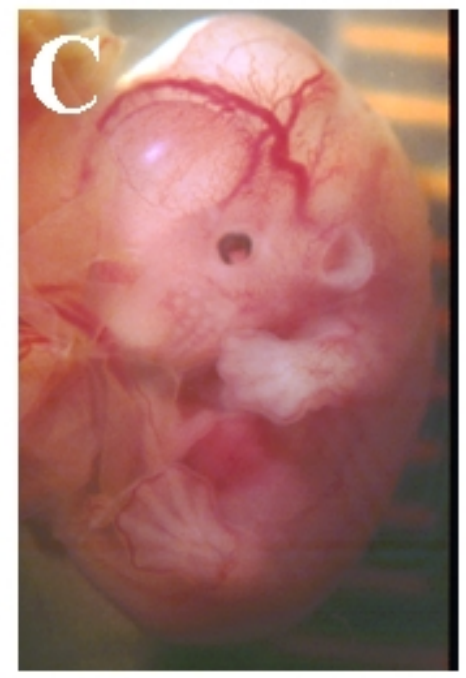

Fig. 5.8 The developing Hesl ${ }^{+-}$ embryos. The embryos are obtained from both $\mathrm{HeSI}^{+/-} \times$ $\mathrm{HeSI}^{+/-}$(bottom row) and $\mathrm{HeSl}^{+/-} \times \mathrm{Bst} /+$ (top row) matings. Top row are E13.5 embryos. Middle and lower rows are E12.5 embryos. A, D, and $\mathrm{F}$ are wildtypes. B, C, E, G show Hes 1 mutant embryos. Several defects can be seen in these examples. B and $\mathrm{C}$ show the same embryo, which has a unilaterally microphthalmic eye. The embryo in $\mathrm{E}$ has an undersized skull and its eye is not as fully developed as that of the wildtype littermate in D, evidenced by the incomplete pigmentation of the mutant's ventral retina. Its limb development also appears delayed. The embryo in $\mathrm{G}$ has unfused and wavy spinal cord, a condition that has been reported to occur in $\mathrm{Hes}^{\mathrm{I}^{--}}$homozygous mutants. 
Bst $x$ Hes 1 F 1 retinal area
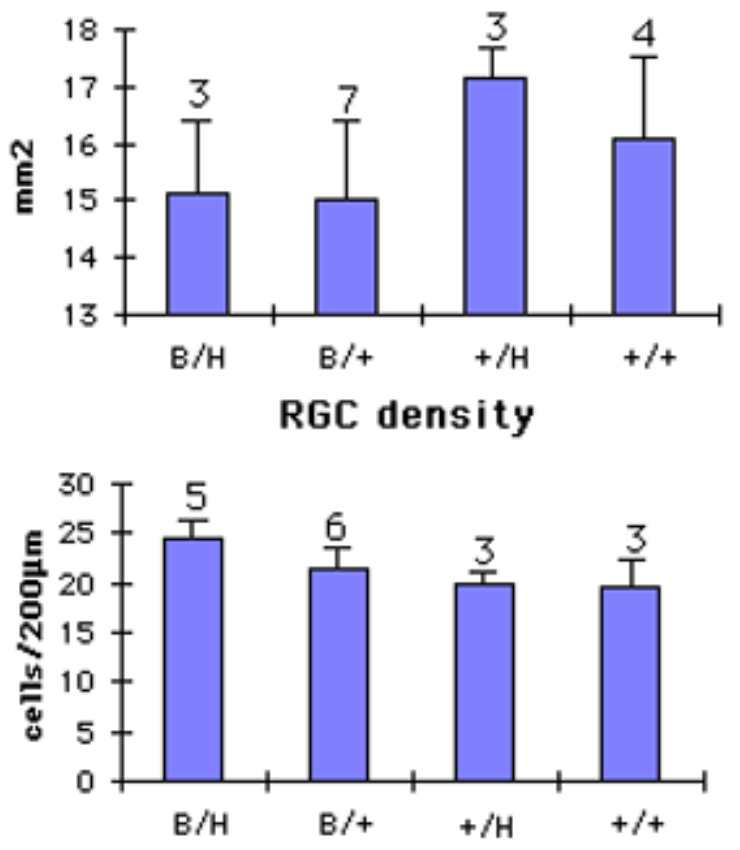

INL cell number

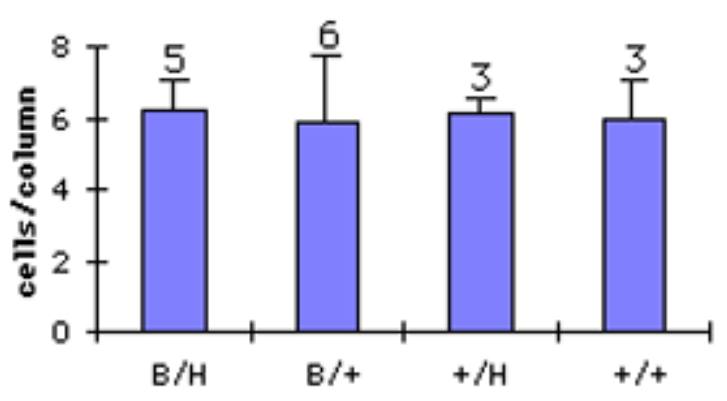

ONL cell number

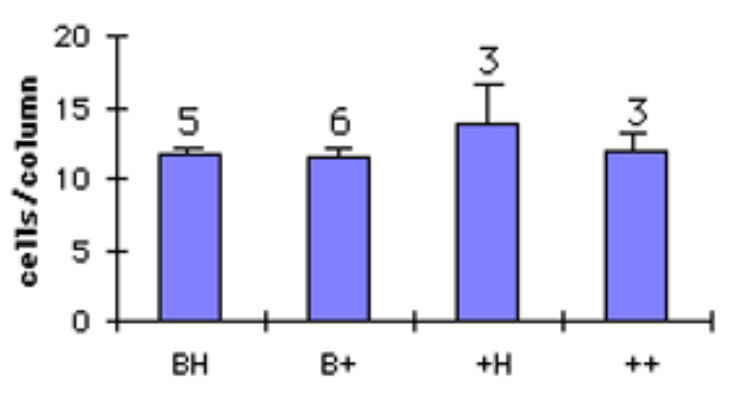

Optic nerve axon number

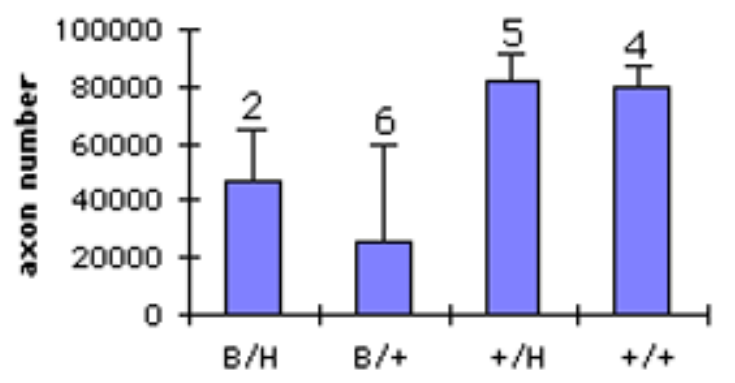

IPL thickness

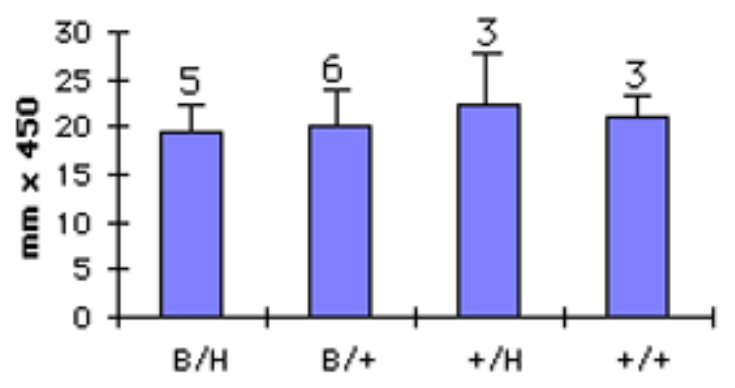

OPL thickness

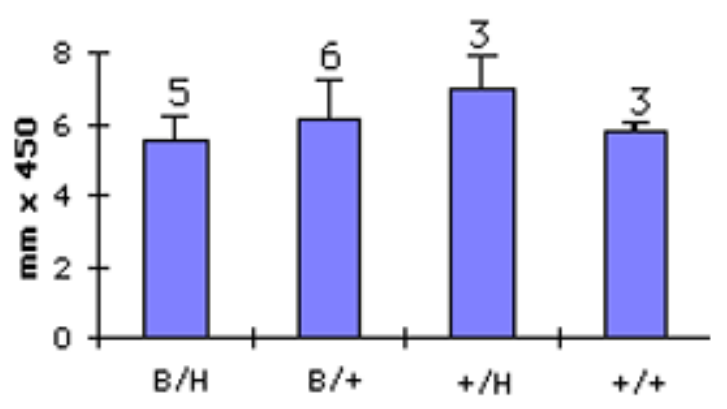

os thickness

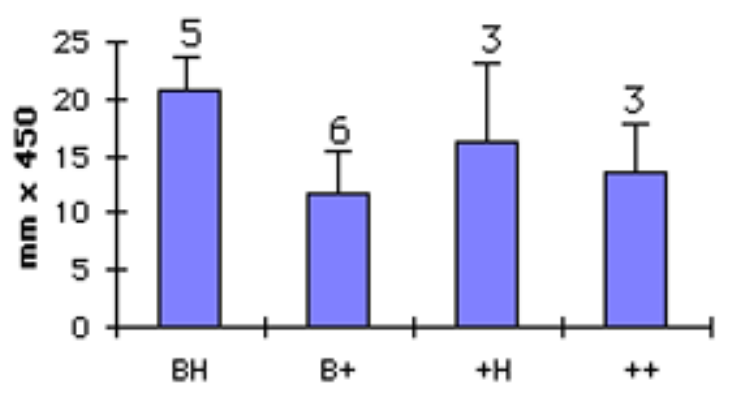

Fig. 5.9 The retinal and optic nerve characteristics of Bst and Hes1 mutant mice. Abrreviations employed: BH

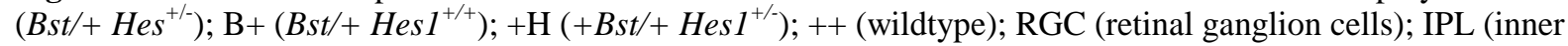
plexiform layer); INL (inner nuclear layer); OPL (outer plexiform layer); ONL (outer nuclear layer); OS (photoreceptor outer segment layer). Cell counts in INL and ONL refer to number of cells in columns spanning the thickness of the respective layers. The differences between groups are typically small. However, B+ usually has the lowest score, while $+\mathrm{H}$ has normal values. The compound mutant $\mathrm{BH}$ scores generally higher thanB + 
Hes $I^{+/+}$having the lowest scores in nearly every aspects measured, edged out slightly by those of the compound mutants. One notable exception is the density of cells in the retinal ganglion cell layer, where $B s t /+$ has the second highest mean, and where the compound mutants have a significantly higher cell density than the other groups (ANOVA, $\mathrm{p}=0.006$ ). However, the actual differences in cell density appear to be too small to compensate for the differences in retinal area; as a group, Bst/ $+\mathrm{Hes} \mathrm{I}^{+/+}$mutants have the lowest number of axons in the optic nerve, the compound mutants have slightly more, and both groups significantly trail the wildtype and $+{ }^{\mathrm{Bst}} /+\mathrm{Hes}^{+/-}$mutants (Fig. 5.9).

\subsection{Discussion.}

Retinal development is dependent upon many regulatory molecules acting in concert with one another to ensure that the correct cellular types are generated at the correct time and position, so as to make proper contact with neighboring cells and structures that could in turn initiate further transformation. The discovery of any particular molecule that perturbs the normal chain of events leading to the formation of the eye provides only a limited clue of the underlying puzzle. Addressing several pieces of the puzzle simultaneously is likely to give complex results, but these results are ultimately invaluable to help us understand the nature of the network within which the individual molecules are a part of - and consequently - the molecules themselves.

Much has been learned about the molecular basis of Drosophila development through the use of compound mutants. For instance, compound heterozygous mutants were employed to elucidate the relationship between ash-1, ash-2 and trithorax during development (Shearn 1989). Compound mutants were also used to characterize deltex as a molecule that interacts with Notch to specify cell fate in many different tissues (Gorman and Girton 1992). In the study of mammalian eye development, the same approach was used to help understand the role of fidget (fi) ocular retardation (or), and microphthalmia (mi), all of which were found to play part in regulating cellular proliferation in the retina (Osipov and Vakhrusheva, 1981; Koniukhov and Sazhina, 1983). The use of compound mutants is a powerful way to either establish or eliminate links between molecules that are suspected to carry out similar functions or act upon the same tissues.

Bst clearly plays a role during early retinal development. We hypothesized that it is involved in promoting cellular differentiation in the neural retina (Tang et al., 1999; see Chapter 4). The results presented here show that cells may indeed be less differentiated in the $B s t /+$ embryonic neural retina than in the wildtype, and that $B s t$ could act as an 
antagonist of the Hes1 gene, which has been characterized as an inhibitor of cellular differentiation within the same tissues during the same developmental period.

5.4.1 Bst disrupts gene expression in the retina.

The most telling clue of $B s t$ as a regulator of cellular differentiation comes from our Pax2 expression analysis. At E11-12, normal Pax2 expression becomes limited to a narrow region of the ventral retina flanking the optic fissure, but in the $B s t /+$, it spreads across almost the entire ventral retina. There are two possible causes: 1) the genetic pathway regulating Pax2 expression is disrupted, and the ectopic expression is the result of a loss of positional cues, or 2) the cells in the ventral retina have not become differentiated enough to stop expressing Pax2. In effect, the retina is behaving "younger". This is consistent with our previous finding that cellular differentiation may be delayed by as many as 2 days in the Bst/+ retina (Tang et al., 1999; see Chapter 4).

The result of our Bst/Krd compound mutant analysis suggests that Bst and Pax2 are indeed functionally linked, but may follow distinct molecular pathways. The fact that given the same genetic background, $B s t /++{ }^{K r d} /+$ mutant mice appear to have improved overall health and retinal development while $+{ }^{B s t} /+K r d /+$ mutants do not, suggest that perhaps two separate modifier gene networks are in part responsible for the mutant retinal phenotype. The possibility that $B s t$ and $\operatorname{Pax} 2$ act through separate pathways is also consistent with the severely disrupted retinal development observed in the compound mutants, as the compound mutation would involve the simultaneous disruption of both chains of events, coalescing into a single outcome that is more than the sum of its parts. We propose that in the compound mutant, the mutant $B s t$ allele causes a delay in cellular differentiation, which, if there are no additional defects, may be neutralized by the action of modifier genes in the hybrid strain background. However, the disrupted Pax2 expression by the $K r d$ allele, which prevents a timely fusion of the optic stalk fissure and the exit of retinal ganglion cell axons, is greatly magnified by the delay caused by the mutant $B s t$ allele, resulting in the complete prevention of ganglion cell axon exit through the optic disk, as well as a host of defects affecting other tissues. Consequently, the surviving mutants invariably lack optic nerves and have dramatically dysmorphic retinas.

It is also evident that many if not all of the defects in the post-natal retina, as well as optic nerve atrophy, are directly attributable to degeneration of the retinal ganglion cell layer. The local thickening of the ONL in the mutant retinas is a curiosity; it is perhaps another product of disrupted positional cues, leading to excess cell proliferation. 
5.4.2 The Bst mutation is probably not a defect in Hes1 expression.

We have previously considered Hesl to be a candidate gene for Bst, since they are both mapped to the same region of mouse Chromosome 16. However, it has been reported that the Heslcoding sequence is not grossly abnormal in the Bst (Tomita et al., 1996; also Dennis Rice, personal communication), ruling out deletion, transposition, and other major defects in the Hes 1 gene as a cause for the Bst mutation. Moreover, Hes 1 mRNA appears to be expressed in normal quantities in the Bst/+ mutant; although an expression time course study would have been necessary to fully eliminate the possibility that Hes Imay be differentially expressed during a limited period of $B s t /+$ development. Given that gene duplication often produces clusters of genes that perform similar functions, the possibility that Bst and Hes 1 are members of the same gene family occupying nearby loci cannot be discounted.

5.4.3 Bst and Hes1 may be antagonistic regulators of differentiation.

In contrast to the results from the $B s t /+\times K r d /+$ study. The results from $B s t /+\times$ Hes ${ }^{+/}$cross mating are more difficult to interpret. This may be partly due to the heterogeneous background of the $\mathrm{C} 3 \mathrm{H}$ line of mice, in which the Hes 1 knockout transgene is introduced, and partly due to a possible direct interaction between Bst and Hes1. If both molecules are regulators of cellular differentiation and are indeed antagonists, then it would follow that in any given compound heterozygous mutant, a range of conditions could exist, from one mutant allele being the more dominant element to the two mutant alleles mutually canceling the effects of one another, thus making the wildtype alleles de facto dominant. Considering that fewer compound mutants than expected are seen among the litters compared to other groups, we surmise that many of the combinations may have been lethal, and that those which survive through birth are necessarily the result of a fortuitously benign combination of the two mutant alleles.

Given the above, it may not be very surprising that another aspect of the Bst/Hes I compound mutants differs markedly from that of the Bst/Krd compound mutants: While the $B s t /+K r d /+$ compound mutants exhibit more defects than either of the two parental mutants, the surviving $B s t /+\mathrm{Hesl}^{+/-}$compound mutants show more defects than their $+{ }^{B s t} /+H e s 1^{+/-}$littermates, but somewhat fewer defects than their Bst/+Hesl ${ }^{+/+}$ littermates, essentially making it an intermediate phenotype.

There are clearly more modifier gene effects present in the $\mathrm{Bst} /+\times \mathrm{HeSl}^{+/-}$cross than in the $B s t /+\times K r d /+$ cross, and some of the modifiers may even be capable of 
causing retinal defects independently of Bst and Hesl. Yet, the retinal phenotype of the compound mutants and their littermates present a consistent pattern, neutral to the genetic background, wherein the surviving compound mutant exhibits fewer defects than one of the parents. This suggests that one of the parental mutations (if they are indeed functional antagonists) may be partially copensating for the other. In this case, $++^{B s t} /+\mathrm{Hesl}^{+/}$are phenotypically indistinguishable from the wildtype, while the compound mutant phenotype is an improvement over that of the $B s t /+\mathrm{Hes}^{+/+}$, thus making the mutant Hes 1 allele the partial "rescuer" of the mutant Bst allele. Although given the above, a case can also be made that the normal Bst allele is a rescuer of the mutant Hesl allele.

It is notable that ganglion cells are present in an unexpectedly high density in the compound mutant retina. Perhaps it is the result of insufficient ocular growth, which restricts the space available to the late-differentiation ganglion cells. It is also possible that since Bst and HesI both affect ganglion cell birth, perhaps through competitive interactions, the animals that survive the effects of the double mutation tend to be those in which the allelic combination favors ganglion cell proliferation.

Taken together, our results are consistent with the hypothesis that Bst is an antagonist of Hesl in the regulation of retinal development. The nature of the interaction between the two genes awaits further characterization, but the interaction is likely to occur over a period of time and within diverse tissues (some of which are evidently vital). The viability of the animal and the functionality of its retina are dependent upon a balanced input from both genes. 
Chapter 6. Conclusions and interpretations.

\subsection{Result summary and discussion.}

The studies described in the preceding chapters show that $B s t$ plays an early role during eye development. The effects of the mutant gene is variable, suggesting that Bst functions in a regulatory pathway involving many other molecules, and that the timed expression and coordination between these molecules determines the outcome of the morphogenetic event they control.

One of the principal findings of our study on embryonic development in the Bst/+ mouse was that the $B s t$ mutation causes a delay in cellular differentiation, which is clearly visible in the neural retina between E10.5 and E12.5. Unlike the developmental delay described in ocular retardation (or) for instance, where a fraction of the retina shows a lengthening cell cycle (Osipov and Vakhrusheva, 1981; Burmeister et al., 1996), the developmental delay in the $B s t /+$ retina is systematic, and does not appear to be caused by slower cellular proliferation. There is also no ectopic cell death in the $B s t /+$ retina to account for its slightly reduced size. Indeed, the $B s t /+$ retina simply appears to develop later than that of the wildtype, but at an essentially normal pace. The only other observable anomaly in the developing Bst/+ eye is the failure of the optic fissure to immediately fuse upon contact. Yet, unlike in other mutations where fusion anomaly is observed, such as in microphthalmia (mi; Hero et al., 1991), where fissure fusion fails to occur due to factors such as abnormal cell proliferation in the pigmented epithelium (PE), optic fissure fusion does eventually occur in the $B s t /+$. In fact, PE fuses normally and ahead of the neural retina in $B s t /+$. Consequently, conditions that lead to microphthalmia (e.g., the collapse of the vitreous) do not exist in the Bst/t.

The effects of $B s t$ in promoting cellular differentiation can be further appreciated through the findings of the compound mutant studies. The ectopic expression of Pax2 in the ventral retina of the Bst/+ mutant at E12.5 is consistent with the hypothesis that these cells are not yet committed to their (correct) fate. When an animal is heterozygous for both the Bst and $K r d$ (Pax2 null) mutant alleles, it seems unable to compensate for either of the alleles and develops no optic nerves. This indicates that while Bst and Pax2 may be involved in independent regulatory pathways controlling cellular differentiation and histogenesis, the developmental program in which they play part is not redundant enough to tolerate the simultaneous disruption of both genes. However, given the high incidence of gene duplication in "higher" metazoans, it is possible that another organism (the human for instance) may be quite capable of attenuating the effects of such a combination 
of mutations. Furthermore, the clear effects of the strain background on the retinal and other developmental phenotypes of the compound mutants from Bst $\times \mathrm{Krd}$ and $\mathrm{Bst} \times \mathrm{Hes} \mathrm{I}$ crosses indicate that $B s t$ likely plays a role in a regulatory pathway involving complex networks of genes. Perhaps like Sonic hedge hog or Notch, Bst is involved in a basic task - a differentiation or proliferation switch - that is common to many regulatory cascades controlling cellular differentiation (Ahmad et al., 1995; Burke and Basler, 1996; Bao and Cepko, 1997; Jensen and Wallace, 1997). Bst may also be differentially and transiently expressed in a defined tissue domain, which changes during the course of development as a function of other gene expressions, such as those of the Pax genes (Chalepakis et al., 1993), allowing Bst to play multiple roles depending on its substrates. Another level of complexity in the matter may involve the possibility that there are multiple alleles of the Bst gene, combinations of which can produce quite different results, as is the case in microphthalmia (mi; Steingrímsson et al., 1994). What does eye development in the Bst and compound mutants reveal regarding these possibilities?

\subsection{The effects of Bst on eye development likely predate optic cup formation.}

Considering the fact that we were able to detect differences among embryos at the earliest stages of optic cup formation (E9.5-10), and that the delayed retinal development seen in later stages is not accompanied by any decrease in the rate of cell proliferation or increased cell death, it is quite possible that the effects of Bst on eye development occur earlier than the formation of the optic cup. This is not surprising, since the pleiotropic effects of the Bst mutation on coat color and skeletal development clearly indicate that the role of $B s t$ is not unique to controlling eye development. The presence of exencephaly and kinky tail are both hallmarks of neuropore closure defects. Among many possibilities, Bst could be involved in the Achaete-Scute, atonal and Notch signaling pathway, which is common to numerous inductive events during development (Artavanis-Tsakonas et al., 1995; Calof, 1995), and thus influence cell fate decision at a number of stages during, and perhaps prior to, the formation of the optic cup. Other candidate regulatory pathways in which Bst may play a role are those controlled by tailless (tll; Hollemann et al., 1998) and $R x$ (Mathers et al., 1997), which have been implicated in the earliest stages of optic vesicle formation. It is also interesting to note that there appears to be a correlation between eye and limb development. For instance, the Drosophila wingless gene affects both eye and wing development, while its many $W n t$ homologues in the vertebrate appear to play important roles in specifying the polarity of developing eye and limb (Parr et al., 
1993). The presence of kinked tail, polydactyly, and exencephaly in the Bst are characteristics of other mutations that affect eye development as well, such as head blebs (Varnum and Fox,1981), extra toes (Xt; Franz and Besecke, 1991), and double foot (Dbf; Lyon et al., 1996). The similarities between $B s t, X t$ and $D b f$ are quite striking. All three genes are semi-dominant on certain strain background, and produce defects of the skull and pelvis that can be attributed to abnormal closure (or failure to close) of both anterior and posterior neuropores. $X t$ produces mild ocular defects, while the eye defects exhibited by $D b f$ are more severe. $X t$ results from a zinc-finger deletion in the Gli3 gene (Schimmang et al., 1992). Gli3 expression in the limb is modulated by Sonic hedgehog (Shh; Marigo et al., 1996). Dbf is located on Chromosome 1, near Pax3; it appears to regulate tissue polarity in the limb. Although the effect of $D b f$ is not dependent upon Shh expression, it may nevertheless play a part in the $S h h$-modulated inductive pathway (Hayes et al., 1998). Conceivably, the same is true of $B s t$ during the early patterning of the limb and the eye. Given the milder phenotype of Bst compared to those of $X t$ and $D b f$, and assuming the strain background is not a significant contributing factor to the differences, Bst may act downstream of $X t$ and $D b f$. It would be interesting to find out if there are protein interaction between these genes once $B s t$ is identified.

\subsection{The meaning of the developmental delay and phenotypic variability.}

There are two aspects of the developmental delay in the $B s t /+$ mutant retina that are intriguing. First, why does the delay result in such variable developmental defects? If cellular proliferation is normal, and the cells are simply being born late throughout the retina, then why are the mutant retinas not a "younger" version of the wildtype retinas? And secondly, given the above, does the delay in optic fissure fusion tell us anything about the nature of the delay?

Clearly, Bst/+ is not just a younger wildtype. For the developmental delay to be disruptive, it must cause events to occur out of synch, such that when the affected tissues are committed to one phase of the histogenetic process (e.g., the commitment of retinoblasts to become ganglion cells in response to certain molecular cues), the environment is either not yet ready to carry out the changes (e.g., the neural retina is not sufficiently developed), or may have already passed the permissive phase (e.g., when many retinoblasts have become committed to other fates). Timing is therefore a strong candidate factor during $B s t$ pathogenesis. We propose the following hypothesis to account for the variable defects occurring in the $B s t /+$ due to timing differences. 
The normal Bst molecule acts in concert with other genes to promote cellular differentiation, and does so within a defined temporal window (Fig. 6.1). In the Bst/+ retina, only half of the Bst gene product is normal, necessitating more time for sufficient Bst-based differentiation cues to build up (presumably to overcome inhibitory factors such as Hes1) to trigger the exit of retinoblasts from the mitotic cycle. In the meantime, the affected retina develops at a normal proliferative rate, which allows it to shadow the wildtype retina in its overall size. Concurrently, cells unaffected by the mutation, such as those of the pigmented retina, progress through differentiation normally, while providing and/or responding to their own complement of morphogenetic cues in a timely fashion. Upon formation of the optic fissure, development of the wildtype retina, as a function of cellular differentiation and proliferation, has progressed to a state where the apparati responsible for either the production of, or the response to, fissure fusion signal molecules are fully in place; consequently fusion occurs without delay. Whereas in the Bst/+ retina, depending on the extent of the developmental delay, the same fusion cues or response elements may be only partially in place or not yet expressed. As a result, fusion does not occur in a timely fashion, and the affected retina may expand beyond normal boundaries, causing retinal folds and associated structural anomalies. The severity of these defects may be proportional to the duration of the abnormal growth period, which begins with the initial contact between opposing fissure margins, and ends with the belated expression of, or response to, fusion cues. The longer the duration of abnormal growth, the more opportunity there is for excess proliferation to occur, culminating in greater structural defects. It is significant that similar variations in optic fissure fusion defects are seen in Hesl-null mouse (Tomita et al., 1996). The disruption of Hes 1 expression results in premature depletion of retinoblasts and impeded retinal growth; optic fissure formation and fusion are thus delayed. We postulate that the extent of fissure fusion defects in the Hesl-null mouse is partially dependent on the length of the delay, just as is the case in the Bst/+. In effect, if Bst and HesI are functional antagonists in a regulatory pathway that defines a controlled equilibrium, should either one fail, the consequence is substantially the same - the loss of equilibrium. Any subsequent developmental event which is dependent upon such an equilibrium could be disrupted in a predictable manner irrespective of the source of the initial perturbation.

In the Bst/Hes 1 compound mutants, we can surmise that many more combinations are possible between the counteractive effects of the two mutant alleles, and many of such combinations may have been lethal. The fortuitous combinations that allowed the 

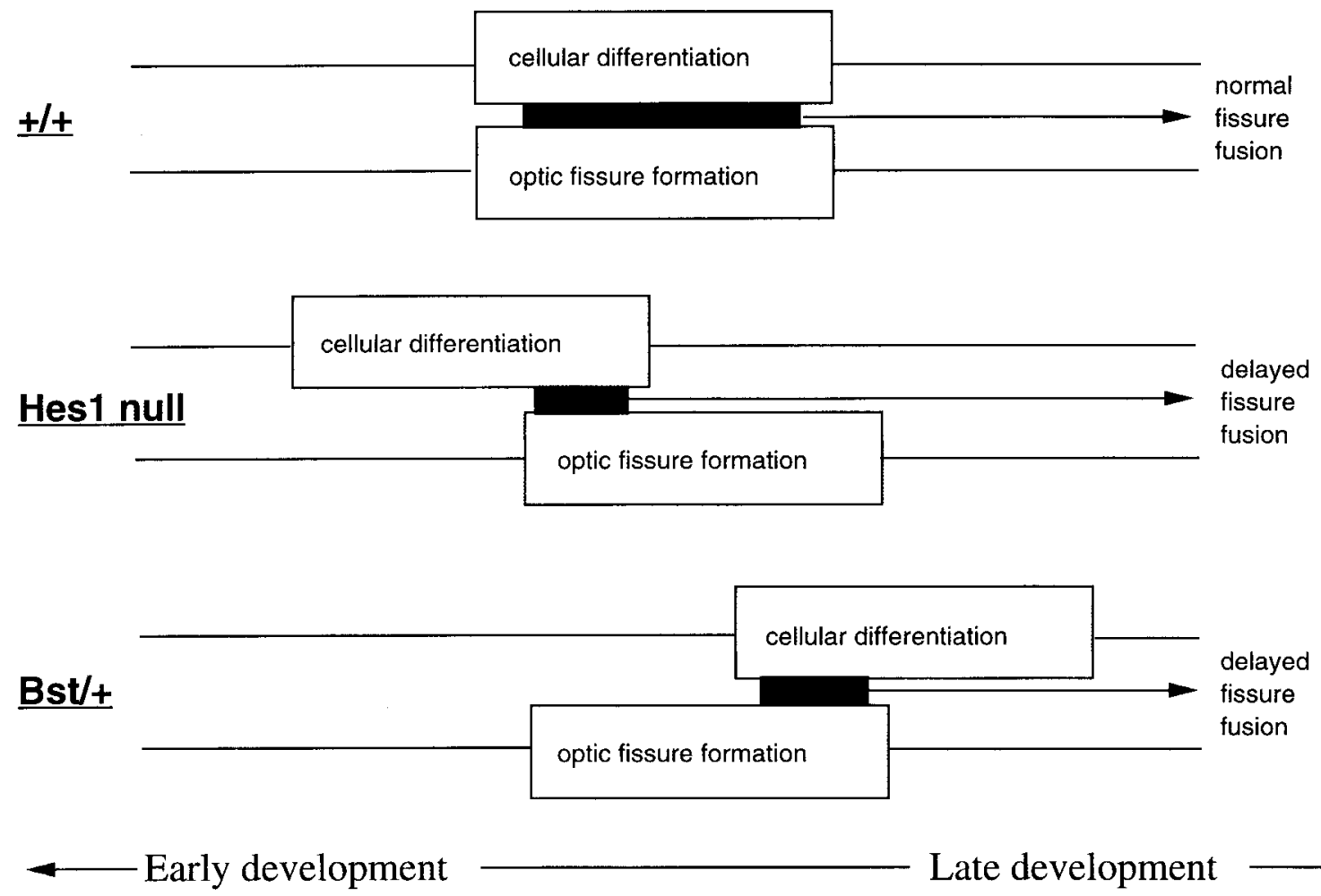

Late development

Fig. 6.1 Hypothetical relationship between cellular differentiation, embryonic fissure formation and fissure fusion. Schematic diagram showing the temporal windows (open blocks) over the course of development within which cellular differentiation and embryonic fissure formation occur, and their combined effect on the fissure fusion quotient (shaded blocks). This quotient delineates the synchrony between the expression of fissure fusion cues and the ready-state of the responding elements. It is a function of the temporal overlap between retinal cellular differentiation and the state of fissure formation, such that the greater the overlap between the temporal windows, the greater the quotient value, and the increased likelihood of normal fissure fusion. Hence, If the two temporal windows are fully synchronized, as is the case in the $+/+$ and the non-symptomatic $B s t /+$, fissure fusion occurs without delay and a normal retina is formed. A slight timing difference between the two beyond a certain threshold may reduce the fusion quotient somewhat and delay fissure fusion, producing small defects in the neural retina. Severe asynchrony could produce a thoroughly dysmorphic retina. This may occur in two ways, as exemplified by development of the Hesl-null and Bst/+ retinas. In the Hesl-null retina, cellular differentiation appears to occur prematurely, which limits the number of retinoblasts available for growth; consequently optic fissure formation occurs late. Such shift in the temporal window alignment results in a lowering of the fissure fusion quotient -- Fusion is delayed. In the Bst/+, cellular differentiation appears delayed, shifting the alignment of the temporal windows in the opposite direction. However, the end result is the same, as delayed cellular differentiation also reduces the fusion quotient (note that in the $B s t /+$, the formation of the optic fissure is generally delayed also as a result of the early growth deficit). The range within which different degrees of misalignment could exist between the temporal windows may be a source of phenotypic variations in the affected retinas. At the extreme, the misalignment may be such that after reaching a certain stage of organogenesis, the embryo becomes no longer viable. Perhaps as a result of this, the proportion of $B s t /+$ neonates in $+/+\times B s t /+$ litters is well short of the expected $50 \%$. 
animals to survive are those in which the effects of one mutant allele may have sufficiently compensated for the disruptive effects of the other, leading to marginal amelioration of the compound mutant phenotype over the worst parental mutant phenotype. In terms of actual cellular events, we could picture that the mutant Hes I allele causes retinoblasts to become prematurely committed to the ganglion cell fate, while the mutant Bst allele delays that commitment, and that in the compound mutant genetic background, the combination of modifier genes are such that premature commitment to cellular differentiation is more tolerable than delay (the Hes1 regulated developmental time window is "larger" than that controlled by $B s t$ ). Consequently $+{ }^{B s t} /+\mathrm{Hes}^{I^{+/-}}$have essentially normal eyes, while $B s t /+\mathrm{HeSl}^{+/+}$do not; and $\mathrm{Bst} /+\mathrm{Hes} \mathrm{I}^{+/}$, when viable, have more prematurely committed cells in the retina than the $B s t /+H e s I^{+/+}$due to the effects of the Hes 1 mutant allele, and thus have somewhat improved retinal phenotype compared to the latter. These speculations can be tested by examining the birthdating pattern in the compound mutants. We have examined two such litters but do not have conclusive results. Due to the inherent variation that exists in the phenotype of the animals, discussed in Chapter 5, it would be difficult to analyze such results, as the differences between groups may be masked by the differences within groups. The difficulty of such a study would be largely mollified by knowing the molecular identity of $B s t$, as we can then examine its expression pattern in the compound mutant along with that of Hesl, and test whether or not there is a correlation between these expressions and how their combination could affect cellular differentiation in the retina.

Another revealing aspect of the Bst mutant phenotype with respect to the gene target is the white belly spot and feet, which is the result of incomplete migration of neural crest-derived melanoblasts. Neural crest cells are pluripotent; both their migration and fate decision are heavily influenced by the substrate along their migration routes (Gilbert, 1985). Consequently, neural crest cells are a prime source for variations and asymmetries that occur during development. It is possible that $B$ st plays a direct role during the fate decision process of neural crest cells, but it is equally possible that $B s t$ only regulates cellular differentiation within the tissues upon which neural crest cells migrate, and indirectly alter the latter's fate. Looking at belly spots of different sizes (Fig. 3.1a), we could either picture abnormally developing neural crests generating fewer melanoblasts than are required to cover the ventral aspect of the mouse, or imagine abnormally differentiating dermatomes that fail to guide melanoblasts to their proper destinations, or induce their differentiation within the correct spatial coordinates. In either scenario, the size of the belly spot could be a reflection of the extent of the disrupted development within the respective tissues. 
While abnormally differentiating or migrating neural crest cells are a potential source for bilateral asymmetry, the side preference seen in the Bst polydactyly phenotype argue that Bst may interact with genes that are asymmetrically expressed. For instance, Shh expression, as well as the expression of a number of conserved signaling molecules, have been shown to be asymmetric in the developing embryo prior to the appearance of morphological asymmetry (Levin, 1997). Recently, using Representational Difference Analysis and Suppressive Subtractive Hybridization, Geschwind and colleagues (1998) have found that numerous genes are asymmetrically expressed in the developing brain. Interestingly, many of the genes they isolated are zinc finger containing transcription factors, including Gli homologues, which are thought to regulate cell fate through the Notch pathway. At least one member of the gene family, Gli3, is known to play a role during both eye and limb development (in $X t$ ). It is conceivable that the asymmetry seen in the Bst occurs through related pathways.

\subsection{The nature of the Bst gene.}

The variable nature of the $B s t$ phenotype has made data analysis somewhat more difficult during these studies, especially when cast against a background of mixed strains in the compound mutant experiments, in which the inherent variability of the other mutant phenotypes compounded the complexity of the outcome. No attempt was made to analyze the results beyond comparing the means using ANOVA, since the purpose of these studies was to detect gross developmental anomalies that can be attributed to the Bst mutation, and not the identification of all contributing factors, which certainly exist. Nevertheless, the variations are themselves clues to the functional nature of $B s t$ and should not be overlooked. As mentioned earlier, the similar but milder phenotype of the $B s t /+$ compared to other mutations that affect ocular, cranial, and skeletal development, such as $X t$ and $D b f$, suggest that it participates in the same, or a parallel regulatory pathway, perhaps in a more redundant or more downstream capacity. These mutations also exhibit variable phenotypes, and many of them have been identified to be transcription factors bearing the basic helix-loop-helix (bHLH) and a leucine zipper motif, such as members of the hairy (Hes), achaete-scute (Mash), and atonal (Math) gene families (Jan and Jan, 1993; Kageyama et al., 1995; Dokucu et al., 1996; Lee, 1997). These molecules are known to act as both positive and negative regulators of cellular differentiation, and likely do so by forming homo- or hetero-dimers between antagonist molecules competing for the same DNA binding domain - a conserved $\mathrm{N}$ box element. The $\mathrm{N}$ box can also be found in the promoter region of some of the bHLH genes, such as 
Hes1, which in this case appears to function in a negative feedback loop (Kageyama et $a l .$, 1995). The function of bHLH molecules is mainly to prevent non-fated cells in the surrounding tissues from acquiring the same fate as the cells expressing these molecules, while promoting the fated cells to enter the committed phase of differentiation. It is conceivable that Bst could be such a molecule, and that it can interact with other bHLH molecules by forming heterodimers with them, just as Mash and Math are thought to do by forming heterodimers with Hes1, and in such a conformation compete against the effects of Hesl homodimers. Indeed, the proximity of the Bst locus with that of Hesl raises the possibility that it may be the product of gene duplication, an event that has occurred numerous times during evolution to generate homeotic gene families (Holland $e t$ al., 1994).

However, Bst does not need to be a bHLH molecule in order to play a role in the same regulatory pathway. While the bHLH molecules act through local interactions (likely via Notch) during histogenesis, their expression is influenced by secreted molecules that regulate the orientation and boundary of the tissue domains, such as members of the wingless (Wnt), and hedgehog $(\mathrm{HH})$ gene families. Consequently, any molecule involved in the inductive pathways, from nuclear proteins to membrane receptors, can all affect the final outcome in a significant manner. For instance, the division abnormally delayed (dally) gene, which codes for a membrane bound heparan sulfate proteoglycan, is a typical receptor for secreted proteins. The dally mutant Drosophila exhibits developmental defects involving the delayed entry of lamina precursor cells into mitosis, and associated disrupted development of the eyes, wings, antennae, and genitalia, which resemble the developmental defects seen in mutations involving homeotic genes (Nakato et al., 1995). Therefore, even if Bst is not a nuclear protein, it can still produce its effects through the pathways we hypothesized.

Another possible molecular pathway in which Bst may play a role is that of retinoic acid (RA). Mutations of some RA receptors have been shown to produce severe retinal dysplasia along with pleiotropic effects in the respiratory, circulatory, digestive, and immune systems (Grondona et al., 1996). Some aspects of developmental defects found in RA receptor mutants are similar to those we see in $B s t /+$ embryos and adults, namely the diversity of affected tissues, variation in the expressivity of the mutant phenotype (Mendelsohn et al., 1994), and a form of polydactyly that shows limb preference (for review, see Morris-Kay and Sokolova, 1996).

Taken together, the results of our studies are consistent with the hypothesis that Bst acts in conjunction with other key regulatory genes, whether they are proneural genes like Hes 1 or boundary genes like Pax2, to control cellular differentiation in the developing 
embryo. With the continued fine-mapping of Bst and the identification of "hot spots" representing candidate modifier genes, we are inching closer towards the isolation and identification of the Bst gene. It is interesting for instance, that Zuo and colleagues have identified several "hot spots" near genes whose known functions make them "unsurprising" modifiers of Bst, namely Pax 6 and Wnt7b (personal communication). Pax6 is involved in the early specification of tissues fated to become the optic cup, while $W n t 7 b$ is involved in determining the dorsal ventral axis of the developing eye. Allelic variations in either gene could very well influence the effects of the Bst gene.

In terms of the origin of the Bst gene, one could only speculate that given the pleiotropic effects of the mutant allele and its apparent effect on the timing of cellular differentiation, its existence likely predated that of the eye. Bst may have been "recruited" into the genetic regulatory networks controlling ocular morphogenesis in much the same way Pax6 is believed to have been recruited. That is, Bst could have been an essential regulator of cellular differentiation and patterning in the head and brain tissues among its earliest roles. The emergence of metazoan eyes through specialization and organization of photosensitive cells within those tissues could only have occurred through gradual changes in the differentiation and patterning programs in which Bst played an important part. Given the fact that genes which perform basic functions tend to be highly conserved, it would be very interesting to learn whether or not a homologue of Bst exists in other vertebrates or perhaps even in Drosophila, and whether these homologues perform similar functions as $B s t$ does in the mouse. 
List of References 
Ahmad, I., Dooley, C. M., and Polk, D. L. (1997). Delta-1 is a regulator of neurogenesis in the vertebrate retina. Dev. Biol. 185, 92-103.

Ahmad, I., Zaqouras, P., and Artavanis-Tsakonas, S. (1995). Involvement of Notch -1 in mammalian retinal neurogenesis: association of Notch -1 activity with both immature and terminally differentiated cells. Mech. Dev. 53, 73-85.

Akazawa, C., Ishibashi, M., Shimizu, C., Nakanishi, S., and Kageyama, R. (1995). A mammalian helix-loop-helix factor structurally related to the product of Drosophila proneural gene Atonal is a positive transcriptional regulator expressed in the developing nervous system. J. Biol. Chem. 270, 8730-8.

Alevizopoulos, A., and Mermod, N. (1996). Antagonistic regulation of a proline-rich transcription factor by transforming growth factor beta and tumor necrosis factor alpha. J. Biol. Chem. 271, 29672-81.

Alexiades, M. R., and Cepko, C. (1996). Quantitative analysis of proliferation and cell cycle length during development of the rat retina. Dev. Dyn. 205, 293-307.

Altmann, C. R., Chow, R. L., Lang, R. A., and Hemmati-Brivanlou, A. (1997). Lens induction by Pax6 in Xenopus laevis. Dev. Biol. 185, 119-23.

Artavanis-Tsakonas, S., Matsuno, K., and Fortini, M. E. (1995). Notch signaling. Science 268, 225-32.

Austin, C. P., Feldman, D. E., Ida Jr., J. A., and Cepko, C. L. (1995). Vertebrate retinal ganglion cells are selected from competent progenitors by the action of Notch . Development 121, 3637-3650.

Axelrod, D., Lerner, D., and Sands, P. J. (1988). Refractive index within the lens of a goldfish eye determined from the paths of thin laser beams. Vision. Res. 28, 57-65.

Bang, A. D., and Goulding, M. D. (1996). Regulation of vertebrate neural cell fate by transcription factors. Curr. Opin. Neurobiol. 6, 25-32.

Bao, Z. Z., and Cepko, C. L. (1997). The expression and function of Notch pathway genes in the developing rat eye. J. Neurosci. 17, 1425-34.

Barnes, R. D. (1987). "Invertebrate Zoology". Saunders, New York. 
Basler, K., Christen, B., and Hafen, E. (1991). Ligand-independent activation of the sevenless receptor tyrosine kinase changes the fate of cells in the developing Drosophila eye. Cell 64, 1069-81.

Belecky-Adams, T., Tomarev, S., Li, H. S., Ploder, L., McInnes, R. R., Sundin, O., and Adler, R. (1997). Pax6, Prox 1, and Chx10 homeobox gene expression correlates with phenotypic fate of retinal precursor cells. Invest. Ophthalmol. Vis. Sci. 38, 1293-303.

Bonini, N. M., Leiserson, W. M., and Benzer, S. (1993). The eyes absent gene: genetic control of cell survival and differentiation in the developing Drosophila eye. Cell 72, $379-95$.

Bonini, N. M., Leiserson, W. M., and Benzer, S. (1998). Multiple roles of the eyes absent gene in Drosophila. Dev. Biol. 196, 42-57.

Bovolenta, P., Mallamaci, A., Puelles, L., and Boncinelli, E. (1998). Expression pattern of cSix3, a member of the Six/sine oculis family of transcription factors. Mech. Dev. 70, 201-3.

Brown, N. A., McCarthy, A., and Wolpert, L. (1991). Development of handed body asymmetry in mammals. Ciba Found. Symp. 162, 182-96.

Bryceson, K. (1981). Focusing of light by corneal lenses in a reflecting superposition eye. J. Exp. Biol. 90, 347-50.

Bulfone, A., Gattuso, C., Marchitiello, A., Pardini, C., Boncinelli, E., Borsani, G., Banfi, S., and Ballabio, A. (1998). The embryonic expression pattern of 40 murine cDNAs homologous to Drosophila mutant genes (Dres): a comparative and topographic approach to predict gene function. Hum. Mol. Genet. 7, 1997-2006.

Burke, R., and Basler, K. (1996). Hedgehog-dependent patterning in the Drosophila eye can occur in the absence of Dpp signaling. Dev. Biol. 179, 360-8.

Burmeister, M., Novak, J., Liang, M. Y., Basu, S., Ploder, L., Hawes, N. L., Vidgen, D., Hoover, F., Goldman, D., Kalnins, V. I., Roderick, T. H., Taylor, B. A., Hankin, M. H., and McInnes, R. R. (1996). Ocular retardation mouse caused by Chx10 homeobox null allele: impaired retinal progenitor proliferation and bipolar cell differentiation. Nat. Genet. 12, 376-84.

Burr, A. H. (1984). Photomovement behavior in simple invertebrates. In "Photoreception and Vision in Invertebrates", ed. M. A. Ali, 179-215. Plenum, New York. 
Callaerts, P., Halder, G., and Gehring, W. J. (1997). PAX6 in development and evolution. Annu. Rev. Neurosci. 20, 483-532.

Calof, A. L. (1995). Intrinsic and extrinsic factors regulating vertebrate neurogenesis. Curr. Opin. Neurobiol. 5, 19-27.

Carthew, R. W., Neufeld, T. P., and Rubin, G. M. (1994). Identification of genes that interact with the sina gene in Drosophila eye development. Proc. Natl. Acad. Sci. U S A 91, 11689-93.

Casarosa, S., Andreazzoli, M., Simeone, A., and Barsacchi, G. (1997). Xrx1, a novel Xenopus homeobox gene expressed during eye and pineal gland development. Mech. Dev. 61, 187-98.

Chalepakis, G., Stoykova, A., Wijnholds, J., Tremblay, P., and Gruss, P. (1993). Pax: gene regulators in the developing nervous system. J. Neurobiol. 24, 1367-84.

Chalepakis, G., Tremblay, P., and Gruss, P. (1992). Pax genes, mutants and molecular function. J. Cell. Sci. Suppl. 16, 61-7.

Chanut, F., and Heberlein, U. (1997). Role of decapentaplegic in initiation and progression of the morphogenetic furrow in the developing Drosophila retina. Development 124, 559-67.

Chaudhuri, A., Hallett, P. E., and Parker, J. A. (1983). Aspheric curvatures, refractive indices and chromatic aberration for the rat eye. Vision. Res. 23, 1351-63.

Chen, R., Amoui, M., Zhang, Z., and Mardon, G. (1997). Dachshund and eyes absent proteins form a complex and function synergistically to induce ectopic eye development in Drosophila. Cell 91, 893-903.

Cheyette, B. N., Green, P. J., Martin, K., Garren, H., Hartenstein, V., and Zipursky, S. L. (1994). The Drosophila sine oculis locus encodes a homeodomain-containing protein required for the development of the entire visual system. Neuron 12, 977-96.

Chisholm, A. D., and Horvitz, H. R. (1995). Patterning of the Caenorhabditis elegans head region by the Pax6 family member vab-3. Nature $377,52-5$.

Cole, M. D., and McMahon, S. B. (1999). The Myc oncoprotein: a critical evaluation of transactivation and target gene regulation. Oncogene 18, 2916-24.

Cripps, M. M., and Nash, D. J. (1983). Ontogeny and adult behavior of mice with congenital neural tube defects. Behav. Neural Biol. 38, 127-32. 
Czerny, T., Halder, G., Kloter, U., Souabni, A., Gehring, W. J., and Busslinger, M. (1999). twin of eyeless, a second Pax6 gene of Drosophila, acts upstream of eyeless in the control of eye development. Mol. Cell 3, 297-307.

Dahl, E., Koseki, H., and Balling, R. (1997). Pax genes and organogenesis. Bioessays 19, $755-65$.

Dawkins, R. (1996). “Climbing mount improbable”, 138-97. W. W. Norton \& Co., New York.

de la Pompa, J. L. (1994). Functional interactions between the gene tetanic and the Shaker gene complex of Drosophila. Mol. Gen. Genet. 244, 205-15.

Della, N. G., Senior, P. V., and Bowtell, D. D. (1993). Isolation and characterisation of murine homologues of the Drosophila seven in absentia gene (sina). Development $117,1333-43$.

Dickson, B. J. (1998). Photoreceptor development: breaking down the barriers. Curr. Biol. 8, R90-2.

Dokucu, M. E., Zipursky, S. L., and Cagan, R. L. (1996). Atonal, rough and the resolution of proneural clusters in the developing Drosophila retina. Development 122, 4139-47.

Dominguez, M., and Hafen, E. (1997). Hedgehog directly controls initiation and propagation of retinal differentiation in the Drosophila eye. Genes. Dev. 11, 3254-64.

Duboule, D. (1994). Temporal colinearity and the phylotypic progression: a basis for the stability of a vertebrate Bauplan and the evolution of morphologies through heterochrony. Dev. Suppl., 135-42.

Edgar, B. A., and Lehner, C. F. (1996). Developmental control of cell cycle regulators: a fly's perspective. Science 274, 1646-52.

Epstein, R., Davisson, M., Lehmann, K., Akeson, E. C., and Cohn, M. (1986). Position of Igl-1, md, and Bst loci on chromosome 16 of the mouse. Immunogenetics 23, 78-83.

Favor, J., Sandulache, R., Neuhauser-Klaus, A., Pretsch, W., Chatterjee, B., Senft, E., Wurst, W., Blanquet, V., Grimes, P., Sporle, R., and Schughart, K. (1996). The mouse $\operatorname{Pax2}(1 \mathrm{Neu})$ mutation is identical to a human $P A X 2$ mutation in a family with renalcoloboma syndrome and results in developmental defects of the brain, ear, eye, and kidney. Proc. Natl. Acad. Sci. U S A 93, 13870-5. 
Fietz, M. J., Concordet, J. P., Barbosa, R., Johnson, R., Krauss, S., McMahon, A. P., Tabin, C., and Ingham, P. W. (1994). The hedgehog gene family in Drosophila and vertebrate development. Dev. Suppl., 43-51.

Fjose, A., Nornes, S., Weber, U., and Mlodzik, M. (1993). Functional conservation of vertebrate seven-up related genes in neurogenesis and eye development. Embo J. 12, 1403-14.

Fortini, M. E., Simon, M. A., and Rubin, G. M. (1992). Signalling by the sevenless protein tyrosine kinase is mimicked by Rasl activation. Nature 355, 559-61.

Fotedar, R., Diederich, L., and Fotedar, A. (1996). Apoptosis and the cell cycle. Prog. Cell Cycle Res. 2, 147-63.

Franz, T., and Besecke, A. (1991). The development of the eye in homozygotes of the mouse mutant Extra- toes. Anat. Embryol. 184, 355-61.

Freund, C., Horsford, D. J., and McInnes, R. R. (1996). Transcription factor genes and the developing eye: a genetic perspective. Hum. Mol. Genet. 5, 1471-88.

Froriep, A. (1906). Die Entwickelung des Auges der Wirbeltiere. In "Handbuch der vergleichenden und experimentellen Entwickelungslehre der Wirbeltiere". ed. O. Hertwig. II/1; 139-266. Verlag von Gustav Fischer, Jena.

Fu, W., and Noll, M. (1997). The Pax2 homolog sparkling is required for development of cone and pigment cells in the Drosophila eye. Genes. Dev. 11, 2066-78.

Fulci, G., and Van Meir, E. G. (1999). p53 and the CNS: tumors and developmental abnormalities. Mol. Neurobiol. 19, 61-77.

Gaul, U., Mardon, G., and Rubin, G. M. (1992). A putative Ras GTPase activating protein acts as a negative regulator of signaling by the Sevenless receptor tyrosine kinase. Cell 68, 1007-19.

Gellon, G., and McGinnis, W. (1998). Shaping animal body plans in development and evolution by modulation of Hox expression patterns. Bioessays 20, 116-25.

Geschwind, D. H., Luganov, M., Karrim, J., and Nelson, S. F. (1998). Finding the differences between the developing cerebral hemispheres using RDA and DNA microarray technology. Soc. Neurosci. Abstr. 398, 3.

Gilbert, S. F. (1985). "Developmental Biology". Sinauer Associates, Inc., Sunderland, Massachusetts. 
Glardon, S., Callaerts, P., Halder, G., and Gehring, W. J. (1997). Conservation of Pax6 in a lower chordate, the ascidian Phallusia mammillata. Development 124, 817-25.

Glaser, T., Lane, J., and Housman, D. (1990). A mouse model of the aniridia-Wilms tumor deletion syndrome. Science 250, 823-7.

Goldsmith, T. H. (1990). Optimization, constraint, and history in the evolution of eyes. $Q$. Rev. Biol. 65, 281-322.

Goodman, C. S. (1994). The likeness of being: phylogenetically conserved molecular mechanisms of growth cone guidance. Cell 78, 353-6.

Gorman, A. L., McReynolds, J. S., and Barnes, S. N. (1971). Photoreceptors in primitive chordates: fine structure, hyperpolarizing receptor potentials, and evolution. Science $172,1052-4$.

Gorman, M. J., and Girton, J. R. (1992). A genetic analysis of deltex and its interaction with the Notch locus in Drosophila melanogaster. Genetics 131, 99-112.

Graw, J. (1996). Genetic aspects of embryonic eye development in vertebrates. Dev. Genet. 18, 181-97.

Grindley, J. C., Davidson, D. R., and Hill, R. E. (1995). The role of Pax6 in eye and nasal development. Development 121, 1433-42.

Grondona, J. M., Kastner, P., Gansmuller, A., Décimo, D., Chambon, P., and Mark, M. (1996). Retinal dysplasia and degeneration in $R A R \beta 2 / R A R g$ compound mutant mice. Development 122, 2173-2188.

Grüneberg, H. (1943). Two new mutant genes in the house mouse. J. Genet. 45, 22-28.

Harrison, R. G. (1920). Experiments on the lens in Amblystoma. Proc. Soc. Exp. Biol. Med. 17: 199-200.

Hamre, K. M., Chepenik, K. P., and Goldowitz, D. (1995). The annexins: specific markers of midline structures and sensory neurons in the developing murine central nervous system. J. Comp. Neurol. 352, 421-435.

Guillemot, F., and Joyner, A. L. (1993). Dynamic expression of the murine Achaete-Scute homologue Mash-1 in the developing nervous system. Mech. Dev. 42, 171-85. 
Habener, J. F., and Stoffers, D. A. (1998). A newly discovered role of transcription factors involved in pancreas development and the pathogenesis of diabetes mellitus. Proc. Assoc. Am. Physicians 110, 12-21.

Hafen, E., Dickson, B., Brunner, T., and Raabe, T. (1993). Genetic dissection of signal transduction mediated by the sevenless receptor tyrosine kinase in Drosophila. Philos. Trans. R. Soc. Lond. B. Biol. Sci. 340, 273-8.

Halder, G., Callaerts, P., Flister, S., Walldorf, U., Kloter, U., and Gehring, W. J. (1998). Eyeless initiates the expression of both sine oculis and eyes absent during Drosophila compound eye development. Development 125, 2181-91.

Halder, G., Callaerts, P., and Gehring, W. J. (1995). Induction of ectopic eyes by targeted expression of the eyeless gene in Drosophila. Science 267, 1788-92.

Halder, G., Callaerts, P., and Gehring, W. J. (1995). New perspectives on eye evolution. Curr. Opin. Genet. Dev. 5, 602-9.

Hanson, I., and Van Heyningen, V. (1995). Pax6: more than meets the eye. Trends Genet. $11,268-72$.

Hayes, C., Brown, J. M., Lyon, M. F., and Morriss-Kay, G. M. (1998). Sonic hedgehog is not required for polarising activity in the Doublefoot mutant mouse limb bud. Development 125, 351-7.

Hero, I. (1990). Optic fissure closure in the normal cinnamon mouse. An ultrastructural study. Invest. Ophthalmol. Vis. Sci. 31, 197-216.

Hero, I. (1989). The optic fissure in the normal and microphthalmic mouse. Exp. Eye Res. 49, 229-39.

Hero, I., Farjah, M., and Scholtz, C. L. (1991). The prenatal development of the optic fissure in colobomatous microphthalmia. Invest. Ophthalmol. Vis. Sci. 32, 2622-35.

Hickman, C. P. (1961). "Integrated Principles of Zoology". The C.V. Mosby Company, St. Louis.

Hill, R. E., Favor, J., Hogan, B. L., Ton, C. C., Saunders, G. F., Hanson, I. M., Prosser, J., Jordan, T., Hastie, N. D., and van Heyningen, V. (1991). Mouse small eye results from mutations in a paired-like homeobox- containing gene [published erratum appears in Nature 1992 Feb 20;355(6362):750]. Nature 354, 522-5. 
Hiromi, Y., Mlodzik, M., West, S. R., Rubin, G. M., and Goodman, C. S. (1993). Ectopic expression of seven-up causes cell fate changes during ommatidial assembly. Development 118, 1123-35.

Holland, P. W., Garcia-Fernandez, J., Williams, N. A., and Sidow, A. (1994). Gene duplications and the origins of vertebrate development. Dev. Suppl., 125-33.

Hollemann, T., Bellefroid, E., and Pieler, T. (1998). The Xenopus homologue of the Drosophila gene tailless has a function in early eye development. Development 125 , 2425-32.

Holt, C. E., and Harris, W. A. (1993). Position, guidance, and mapping in the developing visual system. J. Neurobiol. 24, 1400-22.

Ishibashi, M., Moriyoshi K., Sasai, Y. Shiota, K., Nakanishi, S., and Kageyama, R. (1994). Persistent expression of helix-loop-helix factor HES-1 prevents mammalian neural differentiation in the central nervous system. EMBO J. 13, 1799-1805.

Ishibashi, M., Ang, S. L., Shiota, K., Nakanishi, S., Kageyama, R., and Guillemot, F. (1995). Targeted disruption of mammalian hairy and Enhancer of split homolog-1 (HES-1) leads to up-regulation of neural helix-loop-helix factors, premature neurogenesis, and severe neural tube defects. Genes. Dev. 9, 3136-48.

Jackson, C. G. (1981). Prenatal development of the microphthalmic eye in the golden hamster. J. Morphol. 167, 65-90.

Jan, Y. N., and Jan, L. Y. (1993). HLH proteins, fly neurogenesis, and vertebrate myogenesis. Cell 75, 827-30.

Jarman, A. P., Grell, E. H., Ackerman, L., Jan, L. Y., and Jan, Y. N. (1994). Atonal is the proneural gene for Drosophila photoreceptors. Nature 369, 398-400.

Jensen, A. M., and Wallace, V. A. (1997). Expression of Sonic hedgehog and its putative role as a precursor cell mitogen in the developing mouse retina. Development 124, $363-71$.

Kageyama, R., Sasai, Y., Akazawa, C., Ishibashi, M., Takebayashi, K., Shimizu, C., Tomita, K., and Nakanishi, S. (1995). Regulation of mammalian neural development by helix-loop-helix transcription factors. Crit Rev Neurobiol 9, 177-88.

Kang, D. C., Motwani, M., and Fisher, P. B. (1998). Role of the transcription factor AP-1 in melanoma differentiation. Int. J. Oncol. 13, 1117-26. 
Kaufmann, M. H. (1992). "The atlas of mouse development". Academic Press, San Diego.

Kapousta, N. V., Hitchcock, P. F., Swank, R. T., and Meisler, M. H. (1994). Kidney and retinal defects $(\mathrm{Krd})$, a transgene-induced mutation with a deletion of mouse chromosome 19 that includes the Pax2 locus. Genomics 23, 309-320.

Keller, S. A., Jones, J. M., Boyle, A., Barrow, L. L., Killen, P. D., Green, D. G., Kapousta, N. V., Hitchcock, P. F., Swank, R. T., and Meisler, M. H. (1994). Kidney and retinal defects $(\mathrm{Krd})$, a transgene-induced mutation with a deletion of mouse chromosome 19 that includes the Pax2 locus. Genomics 23, 309-20.

Kerszberg, M. (1996). Accurate reading of morphogen concentrations by nuclear receptors: a formal model of complex transduction pathways. J. Theor. Biol. 183, 95104.

King, K. L., and Cidlowski, J. A. (1998). Cell cycle regulation and apoptosis. Annu. Rev. Physiol. 60, 601-17.

Kirschfeld, K. (1967). Die Projektion der optischen Umwelt auf der Raster der Rhabdomere im Komplexauge von Musca. Exp. Brain. Res. 3, 248-70.

Kollar, E. J., and Fisher, C. (1980). Tooth induction in chick epithelium: expression of quiescent genes for enamel synthesis. Science 207, 993-5.

Koniukhov, B. V., and Sazhina, M. V. (1983). [Interaction of mutant genes fi, or and mi in the morphogenesis of the mouse eye]. Izv Akad Nauk SSSR [Biol], 100-10.

Konyukhov, B. V., and Sazhina, M. V. (1975). Inhibition of DNA synthesis in embryonic mouse retina as a result of gene interaction. Dev. Biol. 45, 1-6.

Land, M. F. (1969). Movements of the retinae of jumping spiders (Salticidae: Dendryphantinae) in response to visual stimuli. J. Exp. Biol. 51, 471-93.

Land, M. F. (1981). Optics and vision in invertebrates. In "Handbook of Sensory Physiology", ed. H. J. Autrum, VII/6B, 471-592. Springer Verlag, Berlin.

Land, M. F. (1984). Crustacea. In "Photoreception and Vision in Invertebrates", ed. M. A. Ali, 401-38. Plenum, New York.

Land, M. F., and Fernald, R. D. (1992). The evolution of eyes. Annu. Rev. Neurosci. 15, $1-29$. 
Lee, J. E. (1997). Basic helix-loop-helix genes in neural development. Curr. Opin. Neurobiol. 7, 12-20.

Levin, M. (1997). Left-right asymmetry in vertebrate embryogenesis. Bioessays 19, 28796.

Levine, E. M., Passini, M., Hitchcock, P. F., Glasgow, E., and Schechter, N. (1997). Vsx1 and $V s x$-2: two $C h x 10$-like homeobox genes expressed in overlapping domains in the adult goldfish retina. J. Comp. Neurol. 387, 439-48.

Liu, I. S., Chen, J. D., Ploder, L., Vidgen, D., van der Kooy, D., Kalnins, V. I., and McInnes, R. R. (1994). Developmental expression of a novel murine homeobox gene (Chx10): evidence for roles in determination of the neuroretina and inner nuclear layer. Neuron 13, 377-93.

Loosli, F., Winkler, S., and Wittbrodt, J. (1999). Six3 overexpression initiates the formation of ectopic retina. Genes. Dev. 13, 649-54.

Lunkes, A., Hartung, U., Magarino, C., Rodriguez, M., Palmero, A., Rodriguez, L., Heredero, L., Weissenbach, J., Weber, J., and Auburger, G. (1995). Refinement of the OPA1 gene locus on chromosome 3q28-q29 to a region of 2-8 cM, in one Cuban pedigree with autosomal dominant optic atrophy type Kjer [letter]. Am. J. Hum. Genet. 57, 968-70.

Lyon, M. F., Quinney, R., Glenister, P. H., Kerscher, S., Guillot, P., and Boyd, Y. (1996). Doublefoot: a new mouse mutant affecting development of limbs and head. Genet. Res. 68, 221-31.

McCarthy, A., and Brown, N. A. (1998). Specification of left-right asymmetry in mammals: embryo culture studies of stage of determination and relationships with morphogenesis and growth. Reprod. Toxicol. 12, 177-84.

Macdonald, R., Barth, K. A., Xu, Q., Holder, N., Mikkola, I., and Wilson, S. W. (1995). Midline signalling is required for $\operatorname{Pax}$ gene regulation and patterning of the eyes. Development 121, 3267-78.

Macdonald, R., and Wilson, S. W. (1996). Pax proteins and eye development. Curr. Opin. Neurobiol. 6, 49-56.

Manak, J. R., and Scott, M. P. (1994). A class act: conservation of homeodomain protein functions. Dev. Suppl., 61-77.

Mann, I. (1964). "The development of the human eye". Gune and Stratton, New York. 
Martín-Partido, G., Rodríguez-Gallardo, L., Alvarez, I. S., and Navascués, J. (1988). Cell death in the ventral region of the neural retina during the early development of the chick embryo eye. Anat. Rec. 222, 272-281.

Mardon, G., Solomon, N. M., and Rubin, G. M. (1994). dachshund encodes a nuclear protein required for normal eye and leg development in Drosophila. Development 120, 3473-86.

Marigo, V., and Tabin, C. J. (1996). Regulation of patched by sonic hedgehog in the developing neural tube. Proc. Natl. Acad. Sci. U S A 93, 9346-51.

Mathers, P. H., Grinberg, A., Mahon, K. A., and Jamrich, M. (1997). The Rx homeobox gene is essential for vertebrate eye development. Nature 387, 603-7.

Mendelsohn, C., Lohnes, D., Décimo, D., Lufkin, T., LeMeur, M., Chambon, P., and Mark, M. (1994). Function of the retinoic acid receptors during development. Development 120, 2749-2771.

Mlodzik, M., Hiromi, Y., Goodman, C. S., and Rubin, G. M. (1992). The presumptive R7 cell of the developing Drosophila eye receives positional information independent of sevenless, boss and sina. Mech. Dev. 37, 37-42.

Monaghan, A. P., Davidson, D. R., Sime, C., Graham, E., Baldock, R., Bhattacharya, S. S., and Hill, R. E. (1991). The Msh-like homeobox genes define domains in the developing vertebrate eye. Development 112, 1053-61.

Morgan, D. O. (1995). Principles of CDK regulation. Nature 374, 131-4.

Morris, S. C. (1994). A palaeontological perspective. Curr. Opin. Genet. Dev. 4, 802-9.

Morriss-Kay, G. M., and Sokolova, N. (1996). Embryonic development and pattern formation. FASEB J. 10, 961-968.

Muntz, W. R. A., and Raj, U. (1984). On the visual system of Nautilus pompilus. J. Exp. Biol. 109, 253-63.

Nakato, H., Futch, T. A., and Selleck, S. B. (1995). The division abnormally delayed (dally) gene: a putative integral membrane proteoglycan required for cell division patterning during postembryonic development of the nervous system in Drosophila. Development 121, 3687-702.

Nilsson, D. E. (1988). A new type of imaging optics in compound eyes. Nature 332, 76-8. 
Nilsson, D. E., and Pelger, S. (1994). A pessimistic estimate of the time required for an eye to evolve. Proc. R. Soc. Lond. B, 256, 53-8.

Ohno, S. (1970). "Evolution by Gene Duplication". Springer Verlag, Heidelberg.

Okano, T., Kojima, D., Fukada, Y., Shichida, Y., and Yoshizawa, T. (1992). Primary structures of chicken cone visual pigments: vertebrate rhodopsins have evolved out of cone visual pigments. Proc. Natl. Acad. Sci. U S A 89, 5932-6.

Oliver, G., and Gruss, P. (1997). Current views on eye development. Trends Neurosci. 20, $415-21$.

Oliver, G., Loosli, F., Koster, R., Wittbrodt, J., and Gruss, P. (1996). Ectopic lens induction in fish in response to the murine homeobox gene Six3. Mech. Dev. 60, 2339.

Olivier, J. P., Raabe, T., Henkemeyer, M., Dickson, B., Mbamalu, G., Margolis, B., Schlessinger, J., Hafen, E., and Pawson, T. (1993). A Drosophila SH2-SH3 adaptor protein implicated in coupling the sevenless tyrosine kinase to an activator of Ras guanine nucleotide exchange, Sos. Cell 73, 179-91.

Osipov, V. V., and Vakhrusheva, M. P. (1981). [Development of microphthalmos in $M i$ wh/mi fi/fi and Mi wh/mi or/or mice]. Arkh. Anat. Gistol. Embriol. 80, 76-82.

Otteson, D. C., Shelden, E., Jones, J. M., Kameoka, J., and Hitchcock, P. F. (1998). Pax2 expression and retinal morphogenesis in the normal and Krd mouse. Dev. Biol. 193, 209-24.

Parr, B. A., Shea, M. J., Vassileva, G., and McMahon, A. P. (1993). Mouse Wnt genes exhibit discrete domains of expression in the early embryonic CNS and limb buds. Development 119, 247-61.

Passini, M. A., Levine, E. M., Canger, A. K., Raymond, P. A., and Schechter, N. (1997). $V s x-1$ and $V s x$-2: differential expression of two paired-like homeobox genes during zebrafish and goldfish retinogenesis. J. Comp. Neurol. 388, 495-505.

Pfeffer, P. L., Gerster, T., Lun, K., Brand, M., and Busslinger, M. (1998). Characterization of three novel members of the zebrafish $\operatorname{Pax} 2 / 5 / 8$ family: dependency of Pax5 and Pax8 expression on the Pax2.1 (noi) function. Development 125, 3063-74. 
Philippe, H., Chenuil, A., and Adouette, A. (1994). Can the Cambrian explosion be inferred through molecular phylogeny? Dev. Suppl. 15-25.

Pignoni, F., Hu, B., Zavitz, K. H., Xiao, J., Garrity, P. A., and Zipursky, S. L. (1997). The eye-specification proteins $S o$ and Eya form a complex and regulate multiple steps in Drosophila eye development [published erratum appears in Cell $1998 \mathrm{Feb}$ 20;92(4):following 585]. Cell 91, 881-91.

Pignoni, F., and Zipursky, S. L. (1997). Induction of Drosophila eye development by decapentaplegic. Development 124, 271-8.

Pines, J. (1994). The cell cycle kinases. Semin. Cancer Biol. 5, 305-13.

Pines, J. (1994). Protein kinases and cell cycle control. Semin. Cell Biol. 5, 399-408.

Quiring, R., Walldorf, U., Kloter, U., and Gehring, W. J. (1994). Homology of the eyeless gene of Drosophila to the Small eye gene in mice and Aniridia in humans. Science $265,785-9$.

Rajan, T. V. (1999). Would Harvey, Sulston, and Darwin get funded today? The Scientist 13(9), 12.

Reh, T. A. (1992). Cellular interactions determine neuronal phenotypes in rodent retinal cultures. J. Neurobiol. 23, 1067-83.

Reh, T. A., and Cagan, R. L. (1994). Intrinsic and extrinsic signals in the developing vertebrate and fly eyes: viewing vertebrate and invertebrate eyes in the same light. Perspect. Dev. Neurobiol. 2, 183-90.

Rice, D. S., Tang, Q., Williams, R. W., Harris, B. S., Davisson, M. T., and Goldowitz, D. (1997). Decreased retinal ganglion cell number and misdirected axon growth associated with fissure defects in Bst/+ mutant mice. Invest. Ophthalmol. Vis. Sci. 38, 2112-24.

Rice, D. S., Williams, R. W., Ward-Bailey, P., Johnson, K. R., Harris, B. S., Davisson, M. T., and Goldowitz, D. (1995). Mapping the Bst mutation on mouse chromosome 16: a model for human optic atrophy. Mamm. Genome 6, 546-8.

Robb, R. M., Silver, J., and Sullivan, R. T. (1978). Ocular retardation (or) in the mouse. Invest. Ophthalmol. Vis. Sci. 17, 468-73. 
Roessler, E., Belloni, E., Gaudenz, K., Jay, P., Berta, P., Scherer, S. W., Tsui, L. C., and Muenke, M. (1996). Mutations in the human Sonic Hedgehog gene cause holoprosencephaly. Nat. Genet. 14, 357-60.

Ruddle, F. H., Bartels, J. L., Bentley, K. L., Kappen, C., Murtha, M. T., and Pendleton, J. W. (1994). Evolution of Hox genes. Annu. Rev. Genet. 28, 423-42.

Ruddle, F. H., Bentley, K. L., Murtha, M. T., and Risch, N. (1994). Gene loss and gain in the evolution of the vertebrates. Dev. Suppl., 155-61.

Rugh, R. (1968). "The mouse - its reproduction and development". Burgess Publishing, Minneapolis.

Sanyanusin, P., Schimmenti, L.A., McNoe, L. A., Ward, T. A., Pierpont, M. E., Sullivan, M. J., Dobyns, W. B., and Eccles, M. R. (1995). Mutation of the PAX2 gene in a family with optic nerve colobomas, renal anomalies and vesicoureteral reflux. Nat. Genet. 9, 358-364.

Sidman, R. L. (1961). Histogenesis of mouse retina studied with thymidine- $\mathrm{H}^{3}$. In "The Structure of the Eye", ed. G. K. Smelser, 487-506. Academic Press, New York.

Silver, J., and Robb, R. M. (1979). Studies on the development of the eye cup and optic nerve in normal mice and in mutants with congenital optic nerve aplasia. Dev. Biol. $68,175-190$.

Sambrook, J., Fritsch, E. F., and Maniatis, T. (1989). "Molecular Cloning: a Laboratory Manual". Cold Spring Harbor Laboratory Press, New York.

Sawamoto, K., Okano, H., Kobayakawa, Y., Hayashi, S., Mikoshiba, K., and Tanimura, T. (1994). The function of argos in regulating cell fate decisions during Drosophila eye and wing vein development. Dev. Biol. 164, 267-76.

Schimmang, T., Lemaistre, M., Vortkamp, A., and Ruther, U. (1992). Expression of the zinc finger gene Gli3 is affected in the morphogenetic mouse mutant Extra-toes (Xt). Development 116, 799-804.

Serikaku, M. A., and O'Tousa, J. E. (1994). sine oculis is a homeobox gene required for Drosophila visual system development. Genetics 138, 1137-50.

Shearn, A. (1989). The ash-1, ash-2 and trithorax genes of Drosophila melanogaster are functionally related. Genetics 121, 517-25. 
Shen, W., and Mardon, G. (1997). Ectopic eye development in Drosophila induced by directed dachshund expression. Development 124, 45-52.

Shimizu, C., Akazawa, C., Nakanishi, S., and Kageyama, R. (1995). MATH-2, a mammalian helix-loop-helix factor structurally related to the product of Drosophila proneural gene Atonal, is specifically expressed in the nervous system. Eur. J. Biochem. 229, 239-48.

Sidman, R. L., Kosaras, B., and Tang, M. (1996). Pigment epithelial and retinal phenotypes in the vitiligo mivit, mutant mouse. Invest. Ophthalmol. Vis. Sci. 37, 10971115 .

Silver, J. (1976). A study of ocular morphogenesis in the rat using $\left({ }^{3} \mathrm{H}\right)$ thymidine autoradiography: evidence for thymidine recycling in the developing retina. Dev. Biol. $49,487-495$.

Silver, J., and Hughes, A. F. (1974). The relationship between morphogenetic cell death and the development of congenital anophthalmia. J. Comp. Neurol. 157, 281-301.

Silver, J., and Hughes, A. F. W. (1973). The role of cell death during morphogenesis of the mammalian eye. J. Morphol. 140, 159-70.

Silver, J., and Sidman, R. L. (1980). A mechanism for the guidance and topographic patterning of retinal ganglion cell axons. J. Comp. Neurol. 189, 101-11.

Sivak, J. G., Hildebrand, T., and Lebert, C. (1985). Magnitude and rate of accommodation in diving and nondiving birds. Vision. Res. 25, 925-33.

Spemann, H. (1938). "Embryonic Development and Induction". Yale University Press, New Haven.

Steingrímsson, E., Moore, K. J., Lamoreux, M. L., Ferre-D'Amare, A. R., Burley, S. K., Zimring, D. C., Skow, L. C., Hodgkinson, C. A., Arnheiter, H., and Copeland, N. G. (1994). Molecular basis of mouse microphthalmia (mi) mutations helps explain their developmental and phenotypic consequences. Nat. Genet. 8, 256-63.

Studzinski, G. P., and Harrison, L. E. (1999). Differentiation-related changes in the cell cycle traverse. Int. Rev. Cytol. 189, 1-58.

Tang, Q., Rice, D. S., and Goldowitz, D. (1999). Disrupted retinal development in the embryonic belly spot and tail mutant mouse. Dev. Biol. 207, 239-55. 
Tang, Q., Howes, N., Chang, B., Davisson, M., and Goldowitz, D. (1997). Retinal development in the Bst/Pax2 double mutant mouse. Soc. Neurosci. Abstr. 256, 10.

Theiler, K. (1972). "The house mouse ". Springer-Verlag, Berlin Heidelberg New York.

Theiler, K., Varnum, D. S., Nadeau, J. H., Stevens, L. C., and Caginaut, B. (1976). A new allele of ocular retardation: early development and morphogenetic cell death. Anat. Embryol. 150, 85-97.

Tomarev, S. I., Callaerts, P., Kos, L., Zinovieva, R., Halder, G., Gehring, W., and Piatigorsky, J. (1997). Squid Pax6 and eye development. Proc. Natl. Acad. Sci. U S A 94, 2421-6.

Tomarev, S. I., Zinovieva, R. D., Weis, V. M., Chepelinsky, A. B., Piatigorsky, J., and McFall-Ngai, M. J. (1993). Abundant mRNAs in the squid light organ encode proteins with a high similarity to mammalian peroxidases. Gene 132, 219-26.

Tomita, K., Ishibashi, M., Nakahara, K., Ang, S. L., Nakanishi, S., Guillemot, F., and Kageyama, R. (1996). Mammalian hairy and Enhancer of split homolog 1 regulates differentiation of retinal neurons and is essential for eye morphogenesis. Neuron 16, 723-34.

Tomita, K., Nakanishi, S., Guillemot, F., and Kageyama, R. (1996). Mash1 promotes neuronal differentiation in the retina. Genes Cells 1, 765-74.

Torres, M., Gomez-Pardo, E., and Gruss, P. (1996). Pax2 contributes to inner ear patterning and optic nerve trajectory. Development 122, 3381-91.

Truslove, G. M. (1962). A gene causing ocular retardation in the mouse, J. Embryol. Exp. Morphol. 10, 652-60.

Urbánek, P., Fetka, I., Meisler, M. H., and Busslinger, M. (1997). Cooperation of Pax2 and Pax5 in midbrain and cerebellum development. Proc. Natl. Acad. Sci. U S A 94, $5703-8$.

van der Meer-de Jong, R., Dickinson, M. E., Woychik, R. P., Stubbs, L., Hetherington, C., and Hogan, B. L. (1990). Location of the gene involving the small eye mutation on mouse chromosome 2 suggests homology with human aniridia 2 (AN2). Genomics 7, $270-5$.

Varnum, D. S., and Fox, S. C. (1981). Head blebs: a new mutation on chromosome 4 of the mouse. J. Hered. 72, 293. 
Vigier, P. (1908). Sur l'existance réelle et le rôle des neurones. La neurone perioptique des Diptères. C. R. Soc. Biol. (Paris) 64, 959-61.

von Salvini-Plawen, L. (1982). On the polyphyletic origin of photoreceptors. In "Visual Cells in Evolution". ed. J. Westfall, 137-154. Raven Press, New York.

von Salvini-Plawen, L., and Mayr, E. (1977). On the evolution of photoreceptors and eyes. Evol. Biol. 10, 207-263.

Wagner, G. P. (1994). Homology and the mechanisms of development. In "Homology: The Hierarchical Basis of Comparative Biology", 273-299. Academic Press.

Walls, G. L. (1942). "The Vertebrate Eye and Its Adaptive Radiation." Cranbrook Press, Michigan.

Walther, C., and Gruss, P. (1991). Pax6, a murine paired box gene, is expressed in the developing CNS. Development 113, 1435-49.

Walther, C., Guenet, J. L., Simon, D., Deutsch, U., Jostes, B., Goulding, M. D., Plachov, D., Balling, R., and Gruss, P. (1991). Pax: a murine multigene family of paired boxcontaining genes. Genomics 11, 424-34.

Wang, S., Sdrulla, A. D., diSibio, G., Bush, G., Nofziger, D., Hicks, C., Weinmaster, G., and Barres, B. A. (1998). Notch receptor activation inhibits oligodendrocyte differentiation. Neuron 21, 63-75.

Weston, J. A. (1970). The migration and differentiation of neural crest cells. $A d v$. Morphog. 8, 41-114.

Wilson, T. G., and Jacobson, K. B. (1977). Mechanism of suppression in Drosophila. V. Localization of the purple mutant of Drosophila melanogaster in the pteridine biosynthetic pathway. Biochem. Genet. 15, 321-32.

Wolpert, L. (1994). Do we understand development? Science 266, 571-2.

Wolpert, L. (1994). Positional information and pattern formation in development. Dev. Genet. 15, 485-90.

Xu, P. X., Woo, I., Her, H., Beier, D. R., and Maas, R. L. (1997). Mouse Eya homologues of the Drosophila eyes absent gene require Pax6 for expression in lens and nasal placode. Development 124, 219-31. 


\section{Vita}

Qing Tang was born in Shanghai, the People's Republic of China in 1966. He attended elementary school in his hometown and subsequently enrolled at Collége Beau Soleil in Villars sur Ollon, Switzerland, where he spent his formative years learning what it was like to be a lycéen. He entered the University of North Carolina at Charlotte in 1984 majoring in Biology, and earned his bachelor's degree in 1989. He came to the lab of Dr. Dan Goldowitz at UT Memphis in 1992 pursuing his interest in the genetic control of development, and eventually chose to study eye development in the mutant mouse Bst. He is married to Ms. Siew Y. Sim, a software engineer in the Bay area. They have a daughter, Hera K.S. Tang, who will turn 4 on the eve of Y2K. After graduation, Qing plans to continue pursuing his research interests in the genetic regulation of development, and avail himself to any opportunity that allows him to contribute to this rapidly advancing field. 